NASA Contractor Report 3791

\title{
Analysis of Airfoil \\ Transitional \\ Separation Bubbles
}

\author{
R. L. Davis and J. E. Carter
}

CONTRACT NAS1-16585

JULY 1984

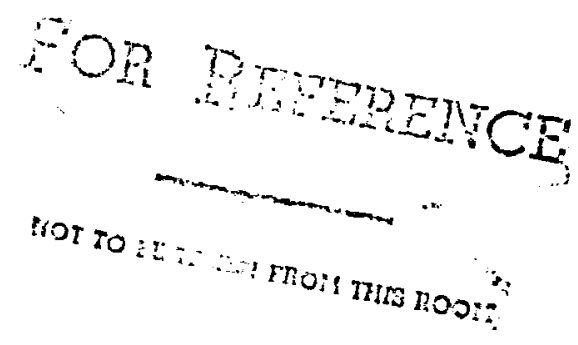

\section{N/SA}



NASA Contractor Report 3791

\section{Analysis of Airfoil \\ Transitional \\ Separation Bubbles}

R. L. Davis and J. E. Carter

United Technologies Research Center

East Hartford, Connecticut

Prepared for

Langley Research Center

under Contract NAS1-16585

\section{NASA}

National Aeronautics

and Space Administration

Scientific and Technical

Information Branch 

TABLE OF CONTENTS

SUMMARY ................................ 1

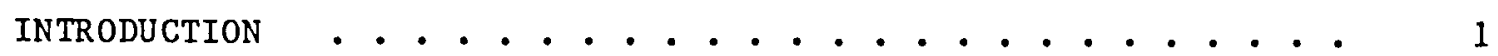

LIST OF SYMBOLS ........................ 3

INVISCID-VISCOUS INTERACTION ANALYSIS . . . . . . . 5

Inviscid Analysis . . . . . . . . . . . 6

viscous Analysis ................... 7

Formulation ................ 7

Windward Differencing .......... 10

Natural Transition/Turbulence Model ...... 12

Interaction Iteration Procedure ........ 16

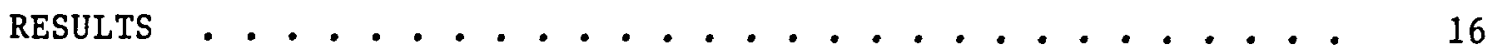

Windward Differencing ............. 16

Gault NACA-0010 Airfoil .......... 16

Gaster Experiment . . . . . . . . . 19

Natural Transition/Turbulence Model ......... 20

Attached Flow ............. 20

Separated Flow .................. 21

CONCLUDING REMARKS ...................... 23

REFERENCES ............................... 25

APPENDIX A - Analysis of the Inviscid Flow Over an Airfoil with a Small Viscous Induced Bump ....... 28 



\section{SUMMARY}

A previously developed local inviscid-viscous interaction technique for the analysis of airfoil transitional separation bubbles, ALESEP (Airfoil Leading Edge Separation), has been modified to utilize a more accurate windward finite difference procedure in the reversed flow region, and a natural transition/turbulence model has been incorporated for the prediction of transition within the separation bubble. Numerous calculations and experimental comparisons are presented to demonstrate the effects of the windward differencing scheme and the natural transition/ turbulence model. Grid sensitivity and convergence capabilities of this inviscid-viscous interaction technique are briefly addressed. A major contribution of this report is that with the use of windward differencing, a second, counter-rotating eddy has been found to exist in the wall layer of the primary separation bubble.

\section{INTRODUCTION}

The prediction of airfoil stall is an engineering problem of considerable importance as it determines one of the major operating limits of an aircraft. The physical mechanisms and different types of airfoil stall are reasonably well understood as evidenced by the excellent article by Tani (Ref. 1) which is nearly twenty years old. Nonetheless, the capability to predict stall has lagged considerably behind this progress due to the overall complexity of the flow, particularly the critical role played by the transition process from laminar to turbulent flow, and the development of reliable and accurate numerical methods for separated flow. It was pointed out by Tani (Ref. 1) that airfoils at moderate incidence angles, prior to either leading-edge stall or thin airfoil stall, experience local separation bubbles just downstream of the peak suction (minimum pressure) region. Therefore, as a start on the development of airfoil stall, the present investigation has been conducted to develop a method for the prediction of closed airfoil separation bubbles.

Figure 1 shows a schematic diagram of an airfoil leading-edge bubble which occurs if the Reynolds number is sufficiently low so that the boundary layer remains laminar up to the minimum pressure point. Downstream of this point, separation occurs almost immediately since laminar boundary layers, in contrast with turbulent flows, are extremely sensitive to adverse pressure gradients. A separation bubble forms in which a recirculating streamline pattern is bounded by a shear layer. Since shear layer flows tend to be highly unstable to flow disturbances, transition from laminar to turbulent flow generally occurs in this shear layer. Further downstream, the turbulent mixing between the shear layer flow with the lower dead air region results in entrainment of higher energy air which energizes the flow near the surface thereby resulting in flow reattachment with subsequent turbulent boundary layer flow downstream. As shown in Fig. 1 , the initial portion of the separation bubble is characterized by a pressure plateau followed by a pressure recovery region after the 
transition process is initiated, but prior to flow reattachment. An increase in incidence causes the bubble to move forward and contract in streamwise extent until the flow no longer reattaches. At this incidence angle, bubble bursting has occurred, thereby resulting in leading edge stall which is accompanied by an abrupt loss of lift since the suction peak has now collapsed with the resultant pressure distribution redistributed in a flattened form over the airfoil chord.

There have been numerous experimental investigations conducted such as the work of Bursnal1 and Loftin (Ref. 2), Gault (Ref. 3), Gaster (Ref. 4), Horton (Ref. 5), Ntim (Ref.6), Evans (Ref. 7), Roberts (Ref. 8), and more recently that of Mueller and Batill (Ref. 9) to provide information on the flow in the neighborhood of transitional separation bubbles. Owen and Klanfer (Ref. 10) deduced from experimental measurements that bubble bursting occurs if the momentum thickness Reynolds number at the laminar separation point is less than 125; similarly, Crabtree (Ref. 11) proposed a criterion based on the pressure rise over the bubble. Later, Horton (Ref. 12) developed a semi-empirical theory based on the experimental measurements of Gaster (Ref. 4) for the growth and bursting of laminar separation bubbles. At the present time airfoil analysis codes, such as the NASA-Lockheed multi-element airfoil code (Ref. 13) and the GRUMFOIL code (Ref. 14), use simple criterions such as these to detect the occurrence of laminar separation bubbles and whether or not bursting occurs. In these analyses, if laminar separation is detected without bursting then the flow is assumed to immediately undergo transition to turbulent flow with a jump discontinuity in the boundary layer parameters such as shape factor and skin friction.

With the recent theoretical developments in boundary layer and inviscid-viscous interaction theory for separated flow, there have been several analytical investigations (Refs. 15-19) conducted to provide a more detailed description of the flow process in a laminar separation bubble. Further progress has been made by Gleyzes, Cousteix, and Bonnet (Ref. 20) in which their interaction analysis utilizes a natural transition model deduced from a correlation based upon amplification of laminar instability waves and free stream turbulence.

The present report is the second in a series on the development of a prediction method for airfoil transitional separation bubbles. In the previous investigation, which was reported in Ref. 21 and subsequently condensed into the paper in Ref. 22, an inverse finite difference boundary layer procedure was iteratively combined with a Cauchy integral representation of the inviscid flow which is assumed to be a locally linear perturbation to a known global viscous airfoil solution. Based on the favorable comparisons which were obtained with experimental data, the major conclusion drawn from this previous work was that this inviscid-viscous interaction model provides an accurate description of transitional separation bubbles provided the transition region is specified a priori. The focus of the current effort has been three-fold; first, to continue to improve this interaction technique through the inclusion of a windward differencing technique in the reversed flow region. Previously, the Reyhner and Flugge 
Lotz (FLARE) (Ref. 23) approximation to the streamwise convection term was used in regions of reversed flow. Only minor changes have occurred in the pressure, skin friction and displacement thickness distributions with the inclusion of windward differencing. It has been found that a more accurate treatment of the convection term through the use of windward differencing has shown that a second, counter-rotating bubble exists in the interior of the main separation bubble. The existence of this secondary bubble structure appears to be insensitive to grid size as shown by numerical tests reported herein. In the second effort, the natural transition turbulence model of McDonald-Fish-Kreskovsky (Refs. 24 and 25) has been included to replace the forced transition model previously utilized. The McDonald-Fish-Kreskovsky transition/turbulence model has been found to predict transition too far downstream in separated and low free stream turbulent flows and hence, it is concluded from the present study that further development of this model is required to enhance its applicability to these flows. Finally, in a third effort, a brief study of the convergence of this interaction scheme has been performed along with additional comparisons with experimental data to further evaluate the scheme. Numerical results indicate that the present inviscid-viscous interaction technique is capable of reducing residuals to desired levels.

\section{LIST OF SYMBOLS}

a

c

$c_{\mathrm{f}}$

$D$

$\tilde{\mathbf{f}}$

F

g

H

$\ell$

I

m

$\mathbf{n}$

N

Structural coefficient

Airfoil chord

Skin friction coefficient

Damping factor applied to mixing and dissipation lengths

Perturbation stream function

Velocity ratio, u/u

Total enthalpy ratio, $\mathrm{H} / \mathrm{H}_{\mathrm{e}}$

Total enthalpy

Mixing length or ratio of local to edge density * molecular viscosity product

Reference length or dissipation length

Perturbation mass flow

Coordinate normal to reference displacement surface

Coordinate measured normal to reference displacement surface from the body surface 


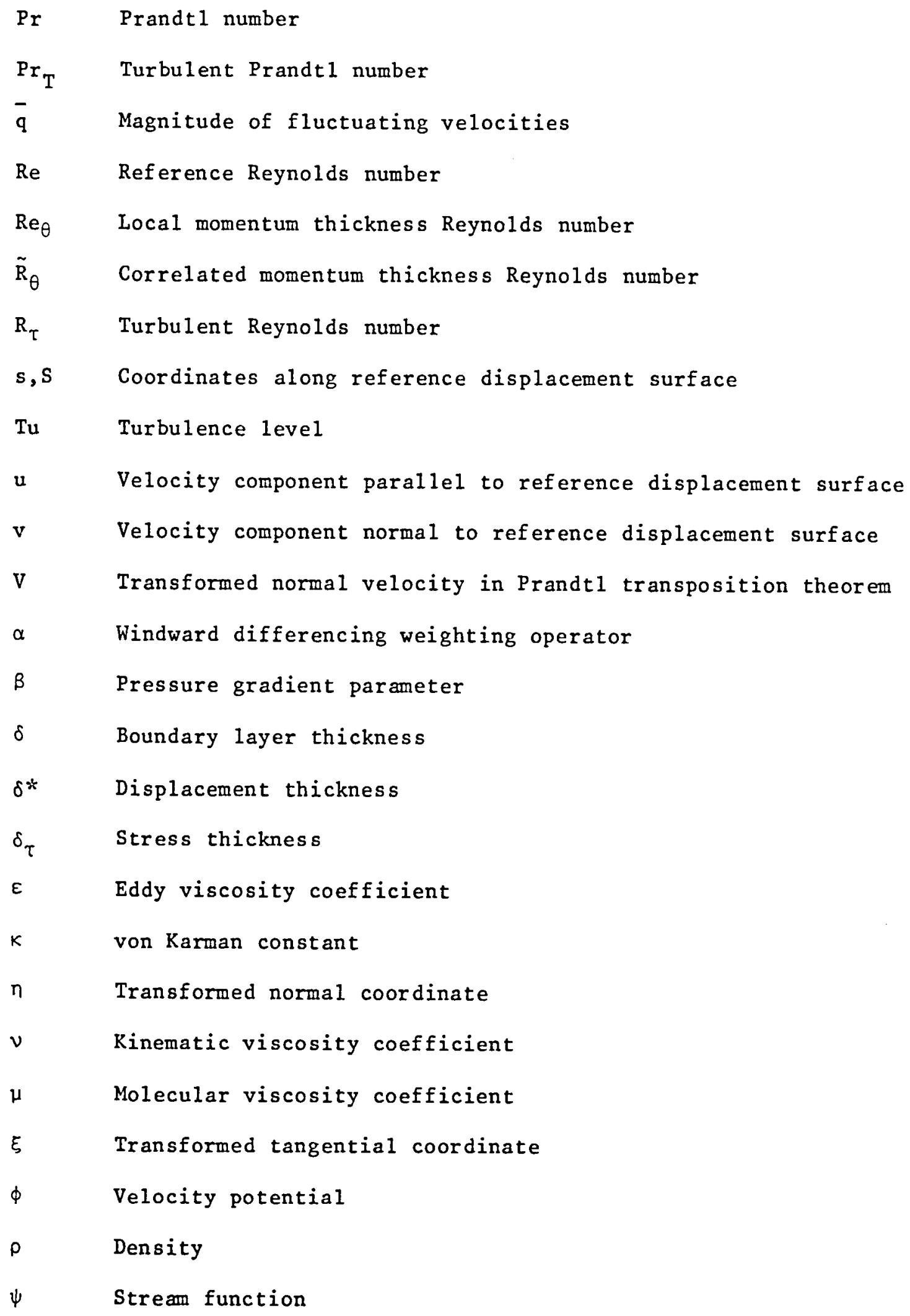




\section{Subscripts}

e Edge of boundary layer

I Inviscid

ref Reference solution

$t_{1} \quad$ Start of transition

$t_{2}$ End of transition

T Turbulent

v Viscous

$\infty \quad$ Free stream

1 Start of interaction region

2 End of interaction region

\section{Superscripts}

- Perturbation quantity

$+\quad$ Inner wall non-dimensionalized coordinate

k Global inviscid-viscous iteration counter

\section{INVISCID-VISCOUS INTERACTION ANALYSIS}

Since the initial development (Refs. 21 and 22) of the transitional inviscid-viscous interaction technique, work has continued to improve this analysis for short transitional separation bubbles. In this analysis, the two-dimensional boundary layer equations are solved in inverse form (Ref. 26) iteratively with an incompressible Cauchy integral perturbation analysis for the inviscid flow. Interaction between the inviscid and viscous solutions is accommodated by using an update formula (Ref. 27) which modifies the specified displacement thickness based upon the differences between the inviscid and viscous velocities at the edge of the boundary layer.

In this section, a review of the inviscid analysis, boundary layer analysis, and the interaction iteration procedure are presented along with a detailed description of the windward differencing scheme and the natural transition/turbulence model which have been used in the present study. 
The local inviscid analysis which was used in the present investigation as well as our previous work (Refs. 21 and 22) assumes that the disturbance field induced by the presence of a transitional separation bubble can be treated as a small disturbance to the global airfoil flow. In Appendix A, a perturbation analysis is used to deduce the governing equations and boundary conditions which describe the local disturbance to the inviscid flow due to the presence of a protrusion on an airfoil. In this analysis, the inviscid flow is split into an inner region which is in the immediate vicinity of the protrusion and an outer region which describes the global flow about the entire airfoil. Under a particular limiting condition for the protrusion height to length ratio, it is shown that to lead order the inner inviscid flow problem reduces to that of a protrusion placed on a flat surface subject to a uniform incoming stream. The magnitude of this uniform stream velocity is the airfoil local surface speed deduced from the global or outer airfoil solution without the presence of the protrusion. It is shown in Appendix A that the governing equation for the perturbation potential for the inner flow region is Laplace's equation subject to the usual small disturbance surface boundary condition at the airfoil surface and uniform flow at large distance from the bump. It is concluded on the basis of this analysis that to lowest order the disturbance field created by the displacement thickness induced by a transitional bubble can be represented by a lineal source distribution placed on the airfoil surface at the transition site. In the future, this technique could be extended to include compressibility effects and thereby develop a rational theory for the disturbance field induced by an airfoil separation bubble in compresible flow.

In Ref. 21 a detailed discussion was presented which showed that if a lineal source distribution is used to represent the local inviscid flow then the surface speed is given by

$$
u^{\prime}(s, 0)=\frac{1}{\pi} \int_{s_{1}}^{52} \frac{\frac{d}{d \xi}\left[u_{e}\left(\delta^{*}-\delta_{r e f}^{*}\right)\right] d \xi}{s-\xi}
$$

The source strength is proportional to the streamwise rate of change of the difference between the computed displacement surface and the reference displacement surface both of which are shown schematically in Fig. 2 .

The Cauchy integral given in Eq. (1) is evaluated from $s_{1}$ to $s_{2}$ which is the region of strong interaction that results from the presence of the local transitional separation bubble. In the present calculations, the reference displacement surface is computed from the GRUMFOIL analysis (Ref. 14) in which instantaneous transition from laminar to turbulent flow is assumed to occur at the predicted laminar separation point. As a result, the interaction displacement surface merges smoothly with the reference displacement surface upstream of the interaction as shown in Fig. 2 . Hence, the lower limit, $s_{1}$, on the Cauchy integral is placed sufficiently far upstream of the interaction region where the source strength is zero. 
The placement of the downstream limit is somewhat more complicated since the interacted displacement surface does not merge into the reference displacement surface downstream of the interaction region. However, this mismatch does not present a problem since, as was demonstrated in Ref. (21), the interacted and reference displacement thicknesses typically are nearly parallel to each other and therefore, do not contribute to the Cauchy integral in this downstream region.

The Cauchy integral given in Eq. (1) is evaluated numerically using a second order scheme developed by Napolitano, et al. (Ref. 28). This numerical procedure permits the use of a nonuniform mesh distribution which was used in the present problem to concentrate grid points near the center of the interaction region in order to adequately resolve the high gradient phenomena which occurs as the flow undergoes transition from laminar to turbulent flow. The same surface grid point distribution was used in both the inviscid and boundary layer analyses thereby avoiding interpolation between these two solutions in the interaction calculations.

\section{Viscous Analysis}

The viscous solution technique used in the present investigation is the inverse boundary layer procedure presented by Carter (Ref. 26) for the analysis of separated flows. Although the inviscid analysis discussed in the previous section has been limited to low speed flow, the boundary layer analysis used in the present study was adapted from earlier work which was for compressible flow. Thus the fully compressible boundary layer analysis is presented here.

\section{Formulation}

The nondimensional boundary layer equations are written as follows in terms of the reference displacement surface coordinate system shown schematically in Fig. 2:

$$
\begin{gathered}
\frac{\partial \rho u}{\partial s}+\frac{\partial \overline{\rho v}}{\partial n}=0 \\
\rho u \frac{\partial u}{\partial s}+\overline{\rho v} \frac{\partial u}{\partial n}=\rho_{e} u_{e} \frac{\partial u_{e}}{\partial x}+\frac{\partial}{\partial n}\left[\mu \frac{\partial u}{\partial n}-\rho \overline{u^{\prime} v^{\prime}}\right] \\
\rho u \frac{\partial H}{\partial s}+\overline{\rho v} \frac{\partial H}{\partial n}=\frac{\partial}{\partial n}\left[\frac{\mu}{\operatorname{Pr}} \frac{\partial H}{\partial n}-\rho \overline{v^{\prime} H^{\prime}}\right. \\
\left.+\mu\left(1-\frac{1}{P_{r}}\right) u \frac{\partial u}{\partial n}\right]
\end{gathered}
$$

The v-component of velocity and the n-coordinate are scaled in the usual manner by $\sqrt{\operatorname{Re}_{\infty C}}$ where $\operatorname{Re}_{\infty c}$ is the Reynolds number based on the free stream flow conditions and the airfoil chord. The boundary conditions 
imposed on these governing equations are:

$$
\begin{gathered}
n=-\delta_{\text {ref }}^{*} u=v=0, \quad H \text { or } \frac{\partial H}{\partial n} \quad \text { SPECIFIED } \\
n \rightarrow \infty \quad u \rightarrow U_{e}, H \rightarrow H_{e}
\end{gathered}
$$

The Reynolds stresses are related to the mean flow by

$$
-p \overline{u^{\prime} v^{\prime}}=\epsilon \frac{\partial u}{\partial n},-p \overline{v^{\prime} H^{\prime}}=\frac{\ell \gamma}{P_{r}} \frac{\partial H}{\partial n}
$$

where $\varepsilon$ is the viscosity coefficient. The transition from laminar to turbulent flow may be modeled using a streamwise intermittency factor, $\gamma(s)$, which varies from 0 to 1 over a specified region.

Werle and Verdon (Ref. 29) showed that it is convenient to transform the boundary layer equations with the Prandt 1 transposition theorem which is given by

$$
s=s, N=n+\delta_{\text {ref }}^{*}, v=v+u \frac{d \delta_{\text {ref }}^{*}}{d s}
$$

where $\mathrm{N}$ is a transformed normal coordinate measured from the airfoil surface perpendicular to the reference displacement surface. In Ref. 29 it is shown that the form of the boundary layer equations is unchanged by this transformation and the same boundary conditions (Eq. (5)) are imposed at $\mathrm{N}=0$.

The development of the inverse formulation begins by transforming the equations, expressed in primitive variables, by the following transformation of the independent variables

$$
\varepsilon=\int_{0}^{x} \rho_{0} \mu_{e} u_{e} d s \quad \eta=\frac{1}{\delta} \int_{0}^{N} \frac{\rho}{P_{e}} d N
$$

which is quite similar to the Levy-Lees transformation. It is helpful to scale the normal coordinate by the displacement thickness in strongly interacting flows since this step insures that the boundary layer thickness is approximately constant in the transformed coordinate. The continuity equation is eliminated by introducing the stream function

$$
\rho u=\frac{\partial \Psi}{\partial N} \quad \rho v=-\frac{\partial \Psi}{\partial S}
$$


The value of the stream function at the boundary layer edge is written in terms of the displacement thickness

$$
\psi-p_{e} u_{0}\left(N-8^{*}\right)
$$

where

$$
\delta^{*}=\int_{0}^{\infty}\left(1-\frac{\rho u}{P_{e} u_{e}}\right) d N
$$

Then with the definitions

$$
m=p_{0} u_{0} 8^{\circ} \quad n=\int_{0}^{\infty}\left(\frac{p_{e}}{\rho}-1\right) d \eta
$$

the edge value of the stream function can be written as

$$
\psi \rightarrow m(\eta-1+h) \text { os } \eta \rightarrow \infty
$$

A ferturbation stream function is defined as

$$
\tilde{f}=\frac{1}{\sqrt{2 \varepsilon}}[\psi-F m(\eta-1+h)] \quad \text { with } \quad F=\frac{u}{U_{0}}
$$

such that $\tilde{f} \rightarrow 0$ as $n \rightarrow \infty$ for a prescribed $m$. Note that in the transformed inverse formulation the perturbation mass flow, $m$, is prescribed and not just the displacement thickness.

Transformation of the compressible boundary layer equations with Eq. (9) and the introduction of the perturbation stream function defined in Eq. (15) results in the following set of governing equations:

$$
\begin{gathered}
\frac{\partial F}{\partial \eta}=\frac{m}{\sqrt{2 \xi}}(1-\eta-h) \frac{\partial F}{\partial \eta} \\
m^{2} F \frac{\partial F}{\partial \xi}-m \frac{\partial}{\partial \xi}\left[\sqrt{2 \xi} \tilde{f}+m F(\eta-1+h) \frac{\partial F}{\partial \eta}=m^{2} \beta\left(g-F^{2}\right)\right. \\
+\frac{\partial}{\partial \eta}\left[\left(1+\frac{\epsilon}{\mu}\right) e \frac{\partial F}{\partial \eta}\right] \\
m^{2} F \frac{\partial g}{\partial \xi}-m \frac{\partial}{\partial \xi}[\sqrt{2 \xi} \tilde{f}+m F(\eta-1+h)] \frac{\partial q}{\partial \eta}= \\
\frac{1}{P r} \frac{\partial}{\partial \eta}\left[v\left(1+\frac{\epsilon}{\mu} \frac{P r}{P r_{1}}\right) \frac{\partial g}{\partial \eta}\right] \\
+\frac{(\gamma-1) M_{0}^{2}}{1+\frac{\gamma-1}{2} M_{0}^{2}} \frac{\partial}{\partial \eta}\left[R\left(1-\frac{1}{P r}\right) F \frac{\partial F}{\partial \eta}\right]
\end{gathered}
$$


where

$$
g=\frac{H}{H_{e}} \quad \beta=\frac{1}{M_{e}} \frac{d M_{e}}{d \xi} \quad \ell=\frac{\rho \mu}{P_{0} \mu_{e}}
$$

Equations (16)-(18) are solved for $F, g, \tilde{f}$ and $\beta$ for a prescribed streamwise distribution of $m$ subject to the following boundary conditions:

$$
\left.\begin{array}{l}
\eta=0 \quad F=\tilde{f}=0 \quad g=g_{w} \text { or }\left.\frac{\partial g}{\partial \eta}\right|_{w} \text { specified } \\
\eta \rightarrow \infty \quad F=g \rightarrow 1 \text { and } \tilde{f} \rightarrow 0
\end{array}\right\}
$$

These equations can also be solved in the direct mode with $\beta$ prescribed and the outer boundary condition $\tilde{\mathrm{f}}=0$ eliminated. In this case, if $\mathrm{m}$ is set equal to $\sqrt{2 \xi}$ then the usual Levy-Lees formulation is deduced with the only difference being that the normal component of velocity has been re-expressed in terms of the stream function. In the inverse case, the unknown pressure gradient parameter is deduced simultaneously with the remainder of the solution. The numerical solution of these equations for the direct and inverse mode is an implicit finite difference technique which is first order accurate in the stream direction and second order accurate in the normal direction. The details of the numerical scheme are presented in Ref. 26. In the next section, the modifications made to this scheme in the reversed flow region are discussed.

\section{Windward Differencing}

In the previous formulation (Ref. 21) of the finite difference representation of the boundary layer equations, the FLARE (Ref. 23) approximation was used in regions of reverse flow (i.e., within the separation bubble') to insure numerical stability. This approximation assumes that streamwise convection is zero in these regions. An objective of the current study has been to assess the accuracy of the FLARE approximation with a more accurate "windward" finite difference scheme in which the flow direction is properly accounted for in the finite difference representation of the streamwise convection terms.

The finite difference cells shown in Fig. 3 are used in the numerical approximation to the boundary layer equations given in Eqs. (16)-(18) for attached flow. A second order accurate central difference operator is applied to the normal gradient terms whereas a one-sided first order accurate operator is applied to the streamwise gradient terms. This first order accurate streamwise differencing scheme is known to be much more stable and free of oscillations than the second order Crank Nicolson difference scheme due to the numerical damping (diffusion) inherent in the lower order accurate scheme. 
Since the boundary layer equations are parabolic, an instability will arise when the solution marching direction is opposite to the flow direction. Reyhner and Flugge Lotz (Ref. 23) have shown that this instability is easily avoided by assuming that the streamwise convection terms are zero in reversed flow regions. For the present equations, the FLARE approximation becomes

$$
\frac{\partial F}{\partial \xi}=\frac{\partial g}{\partial \xi}=0
$$

when $F<0$.

This approximation is particularly attractive since special differencing cells do not have to be utilized in the implementation of the FLARE approximation. Therefore, the differencing cells shown in Fig. 3 may be used everywhere within the boundary layer thus simplifying the numerical scheme.

It is apparent, however, that when this approximation is used, the converged solution of the numerical difference equations is not necessarily the solution to the boundary layer equations since a loss of accuracy due to the negligence of the streamwise convection terms is incurred. As an improvement to the FLARE approximation, a windward finite difference cell such as that shown in Fig. 4 can be used to calculate streamwise gradient terms in reversed flow regions. The streamwise gradients for both attached and separated flow are represented by the weighted difference operator:

$$
\left.\frac{\partial F}{\partial \xi}\right|_{i, j} ^{k}=\alpha \frac{F_{i, j}^{k}-F_{i-1, j}^{k}}{\xi_{i, j}^{k}-\xi_{i-1, j}^{k}}+(1-\alpha) \frac{F_{i+1, j}^{k-1}-F_{i, j}^{k}}{\xi_{i+1, j}^{k-1}-\xi_{i, j}^{k}}
$$

where $\alpha=1 \quad F \geq 0$ attached flow

$=0 \quad F<0$ reversed flow

$k=$ global iteration index

The weighting coefficient, $\alpha$, is usually 1.0 which yields the usual first order backward difference operator for attached flow. For negative streamwise velocities, $\alpha$ is changed to 0 and the difference operator switches to a first order forward difference. Since the marching direction of the numerical scheme is always in the positive (i.e., increasing $i$ ) $\xi$-direction, the information used to calculate the forward difference operator of Eq. (22) must come from the previous global iteration results.

The operator given by Eq. (22) may be applied to all of the streamwise gradient terms including the stream function $\xi$-gradient in Eqs. (17) and (18). Numerical calculations, however, have indicated that the use of this operator on the stream function streamwise gradient leads to an instability as the bubble size increases or as the streamwise grid spacing becomes 
smaller. This instability appears to originate in regions where the normal velocity component is high such as exists at the ends of a large

recirculating region. Application of the weighted difference operator on the stream function has been found to amplify the normal velocity component in these regions. Similar sensitivity to windward differencing on this term was observed in Ref. 30 in which a stream function-vorticity formulation of the boundary layer equations were solved for separated flow. Further research is needed to determine a stable iterative strategy if the $\partial \psi / \partial \xi$ term is to be treated in the same manner as the $\partial F / \partial \xi$ term. Hence, this study has focused on the application of the windward difference operator to the streamwise convection term only.

\section{Natural Transition/Turbulence Model}

In the previous interaction work presented by Carter and Vatsa (Refs. 21 and 22), the Cebeci-Smith turbulence model (Ref. 31) was used to determine the turbulent eddy viscosity coefficient, $\varepsilon$. Either an instantaneous transition or the intermittency function empirically developed by Dhawan and Narasimha (Ref. 32) was used to force the flow from laminar to turbulent. In this earlier report, with the proper selection of transition point and transition length, the effects of the separation bubble on the inviscid loading distribution could be predicted with the interaction technique. Unfortunately, the values of these quantities are not usually known a priori and the solution is sensitive to their specifications.

A focus of the current effort, therefore, has been to incorporate a turbulence model into the interaction technique which has the ability to predict transition automatically. Briley and McDonald (Ref. 15) reported success in predicting transitional separation bubbles with their time dependent Navier-Stokes/boundary layer solver using the McDonald-Fish turbulence model (Ref. 24). This model is based upon the solution of the integral form of the turbulent kinetic energy equation. Since this model has been presented a number of times in previous reports, including the recent critique given by Walker and Werle (Ref. 33), only a brief discussion sion will be presented here. The turbulent kinetic energy equation is

$$
\frac{d}{d s}\left[\frac{\rho_{e} u_{e}^{3}}{2 a_{1}} \phi_{1}\right]=\rho_{e} u_{e}^{3}\left(\phi_{2}-\phi_{3}\right)+E
$$

$$
\text { where } \begin{aligned}
\phi_{1} & \left.=\int_{0}^{\delta_{\tau}} \frac{\rho u}{\rho_{e} u_{e}} \mid\left(\ell \frac{\partial u / u_{e}}{\partial \eta}\right)^{2}+a_{1} y^{f} \frac{\overline{a_{e}{ }^{2}}}{u_{e}^{2}}\right] d \eta, \\
& \phi_{2}=\int_{0}^{\delta} \frac{\rho}{\rho_{e}} l^{2}\left|\frac{\partial u / u_{e}}{\partial \eta}\right|^{3}\left[1-\frac{\ell}{L}\right] d \eta,
\end{aligned}
$$




$$
\begin{gathered}
\phi_{3}=\int_{0}^{\delta_{\tau}} \frac{\rho}{\rho_{e}}\left(\frac{a_{2} a_{3}}{a_{1}}\right)\left[\left(\ell \frac{\partial u / u_{e}}{\partial \eta}\right)^{2}+a_{1} \xi f_{\tau} \frac{\overline{a_{e}^{2}}}{u_{e}^{2}}\right] \frac{1}{u_{e}} \frac{\partial u}{\partial s} d \eta \\
E=\frac{\overline{q_{e}^{2}}}{2}\left[\rho_{e} u_{e} \frac{d \delta}{d s}-\rho_{e} v_{e}\right]
\end{gathered}
$$

where

$$
\begin{gathered}
f_{\tau}=1-\cos \frac{\pi N}{\delta_{\tau}} \\
\gamma=\left\{\begin{array}{l}
0 \text { McDonald-Fish Model } \\
1 \text { McDonald-Kreskovsky-Fish Model }
\end{array}\right\}
\end{gathered}
$$

In the numerical predictions to be presented in the Results Section, the McDonald-Fish-Kreskovsky model (Ref. 25) has been used in attached direct boundary layer calculations and the McDonald-Fish (Ref. 24) model has been used in separated interacting calculations as suggested by Briley and McDonald. For the McDonald-Fish-Kreskovsky model, the value of $\delta_{\tau}$ is defined as the first $N$ location from the edge of the boundary layer where the local shear stress exceeds 2 percent of its maximum value. If $\delta_{\tau}$ is computed to be less than the boundary layer thickness, $\delta$, then $\delta \tau$ is reset to equal $\delta$. For the McDonald-Fish model, $\delta_{\tau}$ is set equal to $\delta$. Structural coefficients, $a_{1}, a_{2}$, and $a_{3}$, have been introduced in terms of the local mixing length, $\ell$, to determine the components of the perturbation velocities.

$$
\begin{gathered}
-\overline{u^{\prime} v^{\prime}}=a_{1}\left\{\bar{q}^{2}-f_{r} \bar{q}_{e}^{2}\right\}=\ell^{2}\left|\frac{\partial u}{\partial N}\right| \frac{\partial u}{\partial N} \\
\overline{u^{\prime 2}}=a_{2} \overline{q^{2}} \\
\overline{v^{\prime 2}}=a_{3} \overline{q^{2}}
\end{gathered}
$$

The total magnitude of the fluctuating velocities is 


$$
\bar{q}^{2}={\overline{u^{\prime}}}^{2}+{\overline{v^{\prime}}}^{2}+{\overline{w^{\prime}}}^{2}
$$

The local mixing length model as given by McDonald and Fish (Ref. 24) and later modified by McDonald and Kreskovsky (Ref. 25) is

$$
\begin{aligned}
\frac{\ell}{\delta} & =D \frac{\ell_{\infty}}{\delta}\left[\tanh \left(\frac{\kappa N}{\delta} \frac{\delta}{\ell \infty}\right)+\frac{\gamma}{2}\left(1-\tanh \frac{\kappa \delta}{\ell \infty}\right)\left(1-\cos \frac{\pi N}{\delta}\right)\right] \text { for } y<\delta \\
& =\frac{\ell \infty}{\delta} \text { for } y \geq \delta
\end{aligned}
$$

Similarly, the dissipation length is

$$
\frac{L}{\delta}=D \frac{L_{\infty}}{\delta} \tanh \left(\frac{\kappa N}{\delta} \frac{\delta}{L_{\infty}}\right)
$$

where $k=0.43$ is the value used for the von Karman constant and $D$ is the damping factor given by

$$
\partial^{2}=\frac{1}{2}\left[1+\operatorname{erf}\left\{\frac{N^{+}-23}{8 \sqrt{2}}\right\}\right]
$$

The inner region scaled normal coordinate is

$$
N^{+}=N \frac{\sqrt{\tau / \rho}}{\nu}
$$

The free stream dissipation length is

$$
\frac{L_{\infty}}{\delta}=0.11
$$

The remaining unknown quantities are the values given to the structural coefficients, $a_{1}, a_{2}$, and $a_{3}$. McDonald and $F i s h$ showed that $a_{1}$ is nearly zero in laminar flow and reaches a value of 0.15 for fully turbulent boundary layers. McDonald and Fish derived a relationship between $a_{1}$ and the turbulent Reynolds number, $R_{\tau}$, where:

$$
R_{T}=\frac{\frac{1}{\delta_{T}} \int_{0}^{\delta_{T}} \nu_{T} d N}{\frac{1}{\delta_{I}} \int_{0}^{\delta_{I}} \nu d N}
$$

where $\nu$ and $\nu_{T}$ are the laminar kinematic and turbulent eddy viscosities, respectively, and $\delta_{I}$ is inner layer thickness defined as the first location from the wall where $v$ is approximately 4 percent of the total effective 
viscosity, $v+v_{T}$. By first converting $R_{\tau}$ into a correlated momentum thickness Reynolas number, $\tilde{\mathrm{R}}_{\theta}$, using an empirical relationship

$$
\begin{aligned}
\tilde{R}_{\theta} & =100 R_{T} 0.22 \text { for } R_{\tau} \leq 1, \\
& =68.26 R_{T}-614.33 \text { for } R_{T} \geq 40
\end{aligned}
$$

the $a_{1}$ structural coefficient may be calculated from the relationship:

where $\begin{aligned} \mathrm{a}_{0} & =0.0115 \\ \mathrm{R}_{\theta_{0}}^{\circ} & =100\end{aligned}$

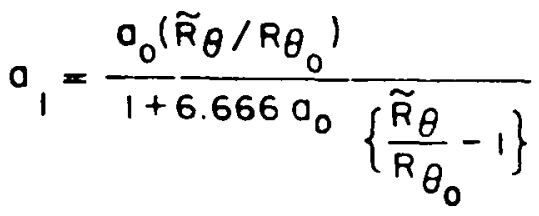

A cubic polynomial which matches values and derivatives at both ends is used to express $\tilde{R}_{\theta}$ in terms of $R_{\tau}$ for $1 \leq R_{\tau}<40$.

Briley and McDonald (Ref. 15) reported that their solution in separated flows was sensitive to the magnitude of the normal stress terms in Eq. (23). Without a modification to structural coefficients, $a_{2}$ and $a_{3}$, the flow upon separating, would not reattach and their solution diverged. For this reason, they allowed the difference in $\mathrm{a}_{2}$ and $\mathrm{a}_{3}$ appearing in $\mathrm{Eq}$. (26) to vary linearly with $R_{T}$ using

$$
\begin{array}{rlrl}
a_{2}-o_{3} & =0.3+0.6\left(1-R_{T}\right) & \text { for } R_{T}<1 \\
& =0.3 \quad \text { for } R_{T} \geq 1
\end{array}
$$

Upon substitution of Eqs. (24)-(42) into the turbulent kinetic energy equation, the free stream mixing length, $l_{\infty}$, may be calculated. The local turbulent eddy viscosity is finally calculated from

$$
\nu_{T}=\frac{-\rho \overline{u^{\prime} v^{\prime}}}{\frac{\partial u}{\partial N}}=\rho \ell^{2}\left|\frac{\partial u}{\partial N}\right|
$$

The transition between laminar and turbulent flow is primarily controlled by the source terms in Eq. (23) and by Eqs. (39)-(41) which determine the magnitude of the structural coefficient $a_{1}$. The source term $E$ which controls the growth of the turbulent kinetic energy is proportional to the free stream turbulence level given by $\overline{q_{e}}$. For convenience $\overline{q_{e}}$ is normalized by the boundary layer edge velocity u

$$
T_{u_{e}}=\left(\frac{\bar{q}_{e}{ }^{2}}{3}\right)^{1 / 2} \frac{1}{u_{e}}
$$


The present analysis is based on a inviscid-viscous iteration technique which was previously presented by Carter (Ref. 27) and is adapted to the present investigation as outlined in Fig. 5. This procedure, which has been referred to as a semi-inverse technique by LeBalleur (Ref. 34), combines an inverse boundary layer technique with a direct inviscid analysis via the update procedure shown in Fig. 5. The key feature of this iteration procedure is the simple update formula

$$
m^{k+1}=m^{k}\left[1+\omega\left(\frac{u_{e_{v}}}{u_{e_{1}}}-1\right)\right]
$$

which permits an inverse boundary layer analysis to be directly linked to an inviscid analysis which accounts for displacement thickness effects. Note that for simplicity the update formula is shown in Fig. 5 with the relaxation factor, $\omega$, set equal to one. It was found by Kwon and Pletcher (Ref. 17) that convergence could be accelerated by making several innerloop passes through the Cauchy integral and the update formula with the boundary layer prediction of the edge velocity, $\mathrm{u}_{\mathrm{e}}$, frozen at its current global iteration value. This technique was used in the present calculations with three inner-loop passes and was found to accelerate the global convergence rate by a factor of three with a 50 percent reduction in computer time as compared to calculations made without this inner iteration.

\section{RESULTS}

\section{Windward Differencing}

In this section, the results obtained with the ALESEP (Airfoil Leading Edge Separation) code using the windward differencing operator of Eq. (22) in the reversed flow regions are compared with the results presented by Carter and Vatsa (Ref. 2l) using the same code but with the FLARE approximation. In all of these calculations, a total of 100 grid points were used in the normal direction in the boundary layer analysis with these points distributed so that the minimum spacing was at the wall. The Cebeci-Smith turbulence model was used with the start and length of transition imposed at the same values as those reported by Carter and Vatsa with the use of the Dhawan and Narasimha intermittency function. Calculations using the windward differencing and FLARE approximations are shown along with experimental data for the Gault (Ref. 3) NACA-0010 airfoil and the Gaster (Ref. 4) series I-IV experiments.

\section{Gault NACA-0010 Airfoil}

The particular Gault case (Ref. 3) which has been analyzed here is for an NACA-0010 airfoil at an 8 degree angle of attack and a chord Reynolds number of $2 \times 10^{6}$ for which a transitional separation bubble occurred in 
the leading edge region. This case has been chosen for the present assessment since the maximum reversed flow velocity, $u / u=-.28$, was the largest of any case analyzed in the previous investigation (Ref. 21). Results obtained from the GRUMFOIL code (Ref. 14) have been used for the reference surface velocity solution and reference displacement thickness distribution. A total of 71 grid points were distributed in the streamwise direction extending between $s / c=0$ to $s / c=.32$ with the minimum spacing located in the transition region. The onset of transition was located at $s / c=.0283$ with a transition length of .0161 .

Figure 6 shows the predicted distributions of pressure, skin friction, and displacement thickness using the windward and FLARE approximations. Comparison of the pressure distributions shows that the only noticeable difference between the windward and FLARE predicted results is a slight decrease in the pressure coefficient at the "breakpoint" (transition point) with the windward scheme. A similar expansion at the end of the pressure plateau region has been observed in the recent experimental data of Jansen and Mueller (Ref. 35) as well as the earlier work by Horton (Ref. 5) on finite swept transitional bubbles. Comparison of the computed results with the experimental pressure data in Fig. 6(a) shows that the inability of the analysis to predict the constant pressure region near the peak suction pressure is still unexplained as it is clearly not affected by the improved differencing procedure used in the reversed flow region. Figures $6(\mathrm{~b})$ and $6(\mathrm{c})$ show that, in general, only small differences exist between the computed skin friction and displacement thickness distributions due to the inclusion of the more accurate windward flow differencing technique. Hence, it is concluded from the analysis of this case, which contains a significant backflow velocity of $u / u_{e}=-.28$, that the FLARE approximation is quite accurate in predicting the overall effects of the transitional separation bubble. Comparison of the windward and FLARE results shows, in this case, only small differences which principally occur in the reversed flow region. These differences, though, are very interesting as the inclusion of windward differencing has revealed a new structure in the recirculating flow region. This change in bubble structure is discussed below.

It is observed in the skin friction distribution in Fig. 6(b) that the calculation performed with the windward scheme in contrast with the FLARE result shows a small region of forward flow $\left(C_{f}>0\right)$ occurs in the interior of the separated flow region. This region occurs at the same location where the surface pressure predicted with the windward scheme shows a slight expansion at the end of the plateau region. Figure 7 shows a comparison of the computed streamlines in the viscous region obtained with the windward and FLARE schemes. Overall, these streamline patterns are very close with the major difference being that the more accurate treatment of the reversed flow region, via the windward scheme, has revealed the existence of a second, counter-rotating bubble inside of the primary separation bubble. To our knowledge this is the first time such a structure has emerged from numerical calculations of the interacting boundary layer equations for closed separation zones on solid surfaces. Physically, such a structure is known to exist in separated flows as 
evidenced by several figures in the excellent compilation on flow visualization recently published by Van Dyke (Ref. 36).

Velocity profiles at eight locations for the windward and FLARE calculations are shown in Fig. 8. with their positions in and around the bubble region denoted by the arrows in Fig. 7. The profile at $\mathrm{s} / \mathrm{c}=.031$ shows the small region of forward flow near the wall which is inside of the secondary bubble predicted by the windward scheme. Except for the region where this inner bubble exists, use of the windward differencing scheme results in slightly lower local velocities within the boundary layer than those calculated by the FLARE approximation. This difference accounts for the slight increase in the displacement thickness found in the windward scheme over that deduced by the FLARE technique for this case.

A grid study has been conducted to determine the double bubble structure sensitivity to the streamwise grid spacing. Only the streamwise grid was varied since the normal mesh of 100 grid points across the boundary layer was thought to be adequate to resolve this secondary bubble. Within the same calculation region $(0<\mathrm{s} / \mathrm{c}<.32)$, a 31 point and a 141 point grid were used in the windward and FLARE schemes. As for the 71 point grid discussed above, the points for these two calculations were distributed nonuniformly with the minimum spacing in the transition region. Figure 9 shows the effect of streamwise grid spacing on the skin friction coefficient using the windward scheme. Despite some differences in the skin friction due to the change in the mesh size, the presence of the inner bubble remains unchanged for these calculations thus demonstrating that its existence is not grid sensitive. Similarly, it was found that the structure of the bubble using the FLARE approximation did not change from that shown in Fig. 7 with these modifications to the streamwise mesh.

Convergence histories for the NACA-0010 calculations using the windward and FLARE schemes are shown in Fig. 10 for the three streamwise grids. A relaxation factor of 0.5 was used in Eq. (45) for both the windward and FLARE calculations for the 141 point grid calculation in order to overcome an iteration instability. A similar situation arose in the earlier work of Carter and Vatsa in which it was found necessary to use underrelaxation on the perturbation mass flow parameter, $m$, in the update procedure to eliminate a similarity instability. No attempt was made in the 141 grid point calculations to optimize the relaxation factor to obtain the fastest convergence rate. Relaxation factors of 1.0 were used in the 31 and 71 point grid calculations. Figure 10 demonstrates that the convergence rate of the windward scheme is nearly the same as that for the FLARE and that both schemes converge monotonically to the desired level of residual. Windward differencing can, therefore, be used with little effect on the convergence rate of the iteration scheme if the fine details of the reversed flow region are of interest. Experience ${ }_{3}$ has indicated that convergence of the edge velocity residuals to $10^{-3}$ produces sufficiently accurate results. 


\section{Gaster Experiment}

A second case with a less intense backflow region has been investigated to compare the results obtained with the windward and FLARE differencing, and in particular, to see if the secondary bubble occurs with the inclusion of the more accurate windward differencing scheme. An experimental investigation of a transitional separation bubble on a flat plate performed by Gaster (Ref. 4) was chosen for this case. The separation bubble was induced by the pressure field generated by the placement of an inverted airfoil near the plate. Interaction calculations using the windward and FLARE approximations were performed for the series I-IV experiment of Gaster. The reference pressure distribution which was used in these interaction calculations was that measured by Gaster on the flate plate with the boundary layer tripped to turbulent flow near the leading edge. The reference displacement thickness was found from a direct boundary layer calculation with the reference pressure distribution prescribed and transition forced to instantaneously occur at the experimental trip location. A uniform mesh consisting of 81 streamwise grid points between $s / L=0.5$ and $s / L=1.5$ was used in the calculations. The Cebeci-Smith turbulence model was used with an assumed instantaneous transition at $\mathrm{s} / \mathrm{L}=1.0$.

Figure 11 shows only minor differences between the windward and FLARE difference schemes for the skin friction and displacement thickness distributions for this case. There are no visible differences in the predicted pressure distributions of the two calculations. Also, the structure of the separation bubble does not change from that predicted with the FLARE approximation in this case. Figure 12 shows a comparison of the velocity profile for the two calculations located at $\mathrm{s} / \mathrm{L}=1.0125$ where the magnitude of the reversed flow velocity is a maximum. Only minor differences exist between the two profiles for this case. The local velocity reaches a $\mathrm{u} / \mathrm{u}_{\mathrm{e}}=-.15$ in the reversed flow region whereas in contrast, the maximum reversed velocity in the Gault NACA-0010 airfoil case, where the impact of windward differencing is greater, is $u / u_{e}=-.28$.

The predicted iso-velocity contours using the windward differencing scheme are compared in Fig. 13 with the experimental contours measured by Gaster. The iso-velocity contours predicted using the FLARE approximation are nearly identical to those shown for the windward scheme. Although good agreement was obtained with the experimental pressure distribution and the separation and reattachment locations as shown in Ref. 21 , the predicted flow field away from the wall differs substantially from the measured iso-velocity contours. It is observed in Fig. 13 that the analysis significantly underpredicts the overall boundary layer growth throughout the bubble. This difference is probably strongly influenced by the assumption of instantaneous transition at $s / L=1.0$ which causes a slope discontinuity in the predicted iso-velocity contours at this location. clearly more work is needed to include a natural transition model in this analysis, which is addressed in the next section of this report. 


\section{Natural Transition/Turbulence Model}

Upon incorporation of the McDonald-Fish-Kreskovsky (MFK) model into the ALESEP code, a series of direct boundary layer calculations using the present finite difference boundary layer procedure were performed to demonstrate the capabilities of the model for attached flows. For separated flow, the modified McDonald-Fish (MF) model was used in the identical manner as Briley and McDonald (Ref. 15) did in their study of transitional separated flow on airfoils. In all of these calculations a total of 100 grid points were placed across the boundary layer with the minimum grid spacing located at the wall.

\section{Attached Flow}

Figure 14 shows the predicted skin friction and displacement thickness with the experimental data taken by Blair (Ref. 37) for transitional, zero pressure gradient flow over a flat plate at a high free stream turbulence level. The free stream velocity was $100.0 \mathrm{ft} / \mathrm{sec}$ and the unit Reynolds number was $5.105 \times 10^{4}$. A total of 54 grid points were distributed unevenly in the streamwise direction with the minimum spacing located at the leading edge of the flat plate. In this case, the free stream turbulence decayed from 7 percent at the initial station to 3.6 percent at $x=94$ inches. Figure 14 shows that the calculated transition region is between $x=2.2$ and $x=6.2$ inches. Since no detailed experimental data exists in the transition region of the flow due to the thinness of the boundary layer, this case demonstrates the accuracy of the boundary layer scheme in turbulent flow using the MFK turbulence model. This case is typical of the high free stream turbulence levels encountered in turbomachinery flows. In external aerodynamics, which is the focus of the present investigation, the free stream turbulence levels are generally substantially lower.

By removing the turbulence generating grids upstream of the flat plate test section, Blair (Ref. 37) measured the boundary layer characteristics for a free stream turbulence level of 0.25 percent. The free stream velocity and Reynolds number were held the same as in the previous case. The computational grid defined in the previous example was used in the predictions for this case as well. Figure 15 shows the comparison between the results obtained with the present direct finite difference boundary layer solution technique using the MFK turbulence model and the experimental data. It is observed that in this case, the predicted location of transition is significantly further downstream than that observed experimentally. Transition is predicted to occur between $x=37.2$ and 55.2 inches whereas transition was measured to occur between $x=27.4$ and 36.0 inches. Delayed transition was also predicted by the ABLE (Analysis of Boundary Layer Equations) finite difference direct boundary layer solver (Ref. 38) using the McDonald-Fish-Kreskovsky transition/turbulence model for this low free stream turbulence case. The accuracy of the present results in the laminar and turbulent regions is demonstrated in Fig. 15 with the inclusion of the known analytical behavior in these respective regions for the skin friction and displacement thickness for a flat plate. 
Excellent agreement is observed in these two regions of the flow thereby confirming the basic approach for fully laminar and turbulent regions while highlighting the transition region shortfall.

The calculations presented in Figs. 14 and 15 were for zero pressure gradient flow over a flat plate. By placing a contoured body opposite to a flat plate section, Sharma, Wagner, Edwards and Blair experimentally induced the boundary layer edge velocity distribution shown in Fig. 16(a) on the flat plate measuring surface. This experiment was conducted in the UTRC Boundary Layer Channel to investigate the boundary layer chracteristics in a flow with a pressure distribution which simulates that in the leading edge region of an airfoil. The inlet to exit velocity ratio was 0.37 and the inlet free stream turbulence level was 7 percent.5 The exit Reynolds number based upon a 36 inch test section was $6.0 \times 10^{5}$. A comparison between the calculated displacement thickness and skin friction of the direct finite difference boundary layer analysis and the experimental data is shown in Figs. 16(b) and 16(c). A total of 200 points were distributed evenly along the flat plate in the boundary layer calculation. Local turbulence levels, $T_{u}$, were calculated from the inlet free stream turbulence and the local to upstream velocity ratio based upon the assumption of frozen turbulence. Although there is no experimental data in the transitiunal region, the agreement between prediction and experiment in the laminar and turbulent regions indicates that the predicted transition region is relatively close to that of the experiment. Transition was predicted to exist between $\mathrm{x}=24.24$ and 29.05 inches with the MFK model.

A non-interacting inverse boundary layer calculation was run on this case to demonstrate the agreement between the direct and inverse boundary layer results using the MFK turbulence model. In order to be consistent between the direct and inverse calculations, the computed values of the perturbation mass flow parameter, m, resulting from the direct finite difference boundary layer calculation described above, were used as boundary conditions in the inverse calculation. The inverse boundary layer calculation reproduced the boundary layer edge velocity distribution shown in Fig. 16(a) demonstrating that the MFK turbulence model has been correctly implemented for inverse boundary layer calculations.

This completes our calculations for attached boundary layer flows. These three cases demonstrate that the MFK transition/turbulence model gives good predicted results for flows with high free stream turbulence levels. However, for low levels of free stream turbulence, the MFK transition model predicts transition too far downstream resulting in a substantial difference between the predicted boundary layer quantities and the corresponding experimental measurements. This same weakness in the transition model is carried over to separated flows as will be demonstrated in the next section.

\section{Separated Flow}

Several calculations for transitional separation bubbles with low free stream turbulence levels have been attempted with the McDonald-Fish (MF) 
turbulence model, modified for separated flow in the same manner as Briley and McDonald (Ref. 15) suggested. Among these calculations are the Gaster case, discussed previously, and the zero angle of attack flow over a NACA $66_{3}-018$ airfoil experimentally studied by Gault (Ref. 3 ). This latter case was computed by Briley and McDonald.

In the present calculations, it has been found that the onset of transition is predicted too far downstream with the MF transition model. The result has a catastrophic effect on the calculation of transitional separation bubbles since the predicted bubble size is now much too large with reattachment frequently not occurring thereby leading to massive sta11. In contrast, Briley and McDonald successfully used the MF transition model in their time dependent Navier-Stokes/boundary layer analysis of the Gault mid-chord transitional bubble case with none of the difficulties which have been encountered in the present study with this transition model. The difference in the present calculations and those of Briley and McDonald is unresolved at the present time; there are significant differences in these two inviscid-viscous interaction formulations but whether or not these differences affect the calculation of the turbulent kinetic energy equation in the transition region will require further study. The delay in transition predicted in the flat plate flow with low free stream turbulence, which was discussed earlier, clearly points to the need for further work in adapting this transitional model to the low free stream turbulence flows in external aerodynamics. It has been found that reattachment can be forced to occur in the analysis of separated flows with the MF transition model by artificially increasing the free stream turbulence level, however. This is demonstrated using the flat plate experiment of Gaster. When the calculation was performed for the free stream turbulence level of 0.25 percent reported by Gaster, the ALESEP code with the MF model predicted that the flow separated from the flat plate with transition and thus reattachment not occurring in the region prior to the downstream end of the calculation located at $s / L=1.5$. By artificially increasing the free stream turbulence level to 4.0 percent, the flow was made to reattach within a reasonable length as shown in Fig. 17 where the predicted pressure, displacement thickness, and skin friction with this level of free stream turbulence are predicted. Local edge turbulence levels were calculated using the local to upstream velocity ratio using the assumption of frozen turbulence. A further increase in turbulence level to 4.5 percent results in a significantly smaller bubble with the pressure distribution not showing the usual pressure plateau region characteristic of separated flows. To further demonstrate the sensitivity of these calculations to the prescribed free stream turbulence level, it was found that a free stream turbulence level of 3.5 percent results in a bubble which has more than doubled in size compared to that at 4.0 percent.

In order to demonstrate the accuracy of the turbulence modeling in the MF model, transition can be forced to occur by simply varying the structural coefficient, $a_{1}$, from 0.0 in laminar flow to 0.15 for turbulent flow over a specified transition length. The variation between these levels is specified with the Dhawan and Narasimha intermittency distribution. The angle of attack for this case is 0.0 degrees and the chord Reynolds number 
is $2.0 \times 10^{6}$. The reference pressure distribution was taken to be the experimental distribution which was obtained at high Reynolds number ( $\operatorname{Re}=$ $1 \times 10^{7}$ ) in which transition naturally occurred before laminar separation could take place. A direct boundary layer calculation was performed from the leading edge of the airfoil to $s / c=0.5$ using the reference pressure distribution as the edge boundary condition. The velocity profile was taken at the $s / c=0.5$ station from the direct calculation to be used as the initial profile for the interacting calculation. The reference displacement thickness was held constant at that value predicted by the direct boundary layer calculation at $s / c=0.5$. A total of 99 points were distributed evenly between $\mathrm{s} / \mathrm{c}=0.5$ and $\mathrm{s} / \mathrm{c}=0.99$ in the interaction calculation. The local edge turbulence level was calculated from a 0.2 percent free stream turbulence level and the local to upstream inviscid veloctiy ratio using the frozen turbulence assumption. Transition was prescribed to occur between $\mathrm{s} / \mathrm{c}=.693$ and $\mathrm{s} / \mathrm{c}=.703$. Figure 18 shows the results of the interaction prediction using this forced transition model on the NACA $66_{3}-018$ airfoil tested experimentally by Gault (Ref. 3). The good agreement between the predicted pressure distribution and Gault's

experimental pressure data shown in Fig. 18(a) demonstrates that the McDonald-Fish turbulence model may be successfully used in the interaction calculation providing that transition is predicted correctly. Figures $18(b)$ and $(c)$ show the predicted displacement thickness and skin friction distributions for this case. The predicted boundary layer velocity profiles at six locations along the airfoil surface using both the FLARE and windward schemes are compared with experimental profiles taken by Gault in Fig. 18(d). For this case where the maximum reversed flow velocity was $\mathrm{u} / \mathrm{u}_{\mathrm{e}}=-.08$, the FLARE and windard schemes produced nearly identical results. The predicted profiles differ from the experimental profiles in the transition region but agree fairly well in the laminar and turbulent regions of flow. As previously shown by Carter and Vatsa (Ref. 21), the predicted results using the interaction technique is quite sensitive to the location of transition. It is shown in Fig. 19 that the sensitivity remains unchanged using the transition model described above. A slight change in the intermittency distribution shown in Fig. 19(a) leads to a major change in the predicted results as shown by a comparison of the experimental velocity profiles at $\mathrm{s} / \mathrm{c}=.725$ with the predicted profiles in Fig. 19(b) using these intermittency distributions. A reduction in transition length of only 0.25 percent chord results in a 22 percent shorter separation bubble and thus an attached turbulent profile at this location. Figure 19 dramatically demonstrates the accuracy which is required of a transition model in order to accurately predict the flow in a transitional separation bubble.

\section{CONCLUDING REMARKS}

The development of an improved airfoil transitional separation bubble analysis has continued through the inclusion of a proper finite difference technique in the reversed flow region and the incorporation of a natural transition/turbulence model to predict the onset of transition within the separation bubble. This introduction of a windward finite difference 
procedure into the present inviscid-viscous technique has been found to be stable and inexpensive in terms of additional computational time over that with the Reyhner-Flugge Lotz (FLARE) approximation. It has been found for flows with reversed flow velocities up to 28 percent of the boundary layer edge velocity that comparable results for the surface pressure, skin friction, and displacement thickness are predicted by the windward and FLARE schemes. However, the use of the more accurate windward difference scheme has revealed a major structural change in the interior of the separation bubble. A counter-rotating region under the primary transitional separation bubble can emerge with the use of windward differencing which has not previously been observed with the FLARE approximation. Numerical tests were performed to indicate that the existence of this secondary structure is not sensitive to grid spacing. The differences in bubble structure and boundary layer characteristics between the windard and FLARE schemes are proportional to the size of the reversed flow region and the magnitude of the reversed flow velocity within the bubble. Convergence studies also indicate that with both the windward and FLARE schemes, the present inviscid-viscous coupling procedure is capable of reducing residuals to desired levels. The McDonald-Fish-Kreskovsky natural transition turbulence model has been incorporated into the interaction code for the prediction of transition within the separation bubble. A number of cases are presented which demonstrate that this model predicts transition too far downstream in separated and low free stream turbulent flows. Sensitivity studies are reported which indicate that modifications to this natural transition model are required for these flows. 


\section{REFERENCES}

1. Tani, I.: Low Speed Flows Involving Bubble Separations, Progress in Aeronautical Sciences, Vol. 5, Pergammon Press, 1964.

2. Bursna11, W. J. and Loftin, L. K., Jr.: Experimental Investigation of Localized Regions of Laminar-Boundary-Layer Separation, NACA TN 233, April 1951 .

3. Gault, D. E.: An Experimental Investigation of Separated Laminar Flow, NASA TN 3505, September 1955.

4. Gaster, M.: The Structure and Behavior of Laminar Separation Bubbles, AGARD Conference Proceedings 4, pp. 819-854, 1966.

5. Horton, H. P.: Laminar Separation Bubbles in Two and Three Dimensional Incompressible Flow, Ph.D. Thesis, University of London, (Queen Mary College), 1968.

6. Ntim, B. A.: A Theoretical and Experimental Investigation of Separation Bubbles, Ph.D. Thesis, Queen Mary College, 1969.

7. Evans, B. J.: Effects of Free-Stream Turbulence on Blade Performance in a Compressor Cascade. Ph.D. Thesis, University of Cambridge, 1971.

8. Roberts, W. B.: A Study of the Effect of Reynolds Number and Laminar Separation Bubbles on the Flow Through Axial Compressor Cascades, $\mathrm{Ph} . \mathrm{D}$. Thesis, Von Karman Institute, 1973.

9. Mueller, T. J. and Batil1, S. M.: Experimental Studies of the Laminar Separation Bubbles on a Two-Dimensional Airfoil at Low Reynolds Numbers, AIAA Paper No. 80-1440, 1980.

10. Owen, P. R. and Klanfer, L.: On the Laminar Boundary Layer Separation from the Leading Edge of a Thin Airfoil, Aeronautical Research Council, Britain, Current Paper 220, 1955.

11. Crabtree, L. F.: Effects of Leading Edge Separation on Thin Wings in Two-Dimensional Incompressible Flow, Journal of Aeronautical Sciences, Vo1. 24, pp. 597-604, August 1957 .

12. Horton, H. P.: A Semi-Empirical Theory for the Growth and Bursting of Laminar Separation Bubbles, Aeronautical Research Council, Britain Current Paper 1073, 1967.

13. Stevens, W. A., Goradia, S. H. and Braden, J. A.: Mathematical Model for Two-Dimensional Multi-Component Airfoils in Viscous Flows, NASA CR-1843, July 1971 . 
14. Melnik, R. E., Chow, R. and Mead, H. R.: Theory of Viscous Transonic Flow Over Airfoils at High Reynolds Number, AIAA Paper 77-680, June 1977 .

15. Briley, W. R. and McDonald, H.: Numerical Prediction of Incompressible Separation Bubbles, J. Fluid Mechanics, Vol. 69, Part 4, pp. 631-656, 1975 .

16. Crimi, P. and Reeves, B. L.: Analysis of Leading Edge Separation Bubbles on Airfoils, AIAA J., Vol. 14, No. 11, pp. 1548-1555, November 1976.

17. Kwon, 0. K. and Pletcher, R. H.: Prediction of Incompressible Separated Boundary Layers Including Viscous-Inviscid Interaction, J. Fluids Engineering, Vo1. 101, pp. 466-472, December 1979.

18. Gleyzes, C., Cousteix, J. and Bonnet, J. L.: Bulbe de Decollment Laminaire Avec Transition--Essai de Prevision Avec Coupleze Local, AGARD, Conference in Computation of Viscous-Inviscid Interactions, No. 21, AGARD CP-291, October 1980 .

19. Cebeci, T. K., Stewartson, K., and Williams, P. G.: Separation and Reattachment Near the Leading Edge of a Thin Airfoil at Incidence, AGARD Conference on Computation of Viscous-Inviscid Interactions, No. 20, AGARD CP-291, October 1980.

20. Gleyzes, C., Cousteix, J. and Bonnet, J. L.: A Calculation Method of Leading Edge Separation Bubbles, Second Symposium on Numerical and Physical Aspects of Aerodynamic Flows, California State University, January 17-20, 1983 .

21. Carter, J. E. and Vatsa, V. N.: Analysis of Airfoil Leading Edge Separation Bubbles, NASA CR-165935, May 1982.

22. Vatsa, V. N. and J. E. Carter: Analysis of Airfoil Leading Edge Separation Bubbles, AIAA Paper No. 83-0300, January 1983.

23. Reyhner, T. A. and Flugge Lotz, I.: The Interaction of a Shock Wave with a Laminar Boundary Layer, Int. Journal of Non-Linear Mech., Vol 3 , No. 2, June 1968, pp. 173-179.

24. McDonald, H., and Fish, R. W.: Practical Calculation of Transitional Boundary Layers, Int. J. Heat Mass Transfer, Vol. 16, pp. 1729-1944, 1973.

25. McDonald, H. and Kreskovsky, J. P.: Effect of Free Stream Turbulence on the Turbulent Boundary Layer, Int. J. Heat Mass Transfer, Vo1. 17, Pp. 705-716, 1974.

26. Carter, J. E.: Inverse Boundary-Layer Theory and Comparison With Experiment, NASA TP-1208, September 1978. 
27. Carter, J. E.: A New Boundary Layer Inviscid Iteration Technique for Separated Flow, AIAA Paper 79-1450, July 22-23, 1979.

28. Napolitano, M., Werle, M. J. and Davis, R. T.: Numerical Solutions of the Triple-Deck Equations for Supersonic and Subsonic Flow Past a Hump, University of Cincinnati, Report No. AFL 78-6-42, June 1978.

29. Werle, M. J. and Verdon, J. M.: Viscid-Inviscid Interaction for Symetric Trailing Edges, Naval Air Systems Command, Contract Report N00019-78-C-0604, January 1980.

30. Carter, J. E.: Inverse Solution for Laminar Boundary-Layer Flows With Separation and Reattachment, NASA TR R-447, 1975.

31. Cebeci, T. and Smith, A. M. 0.: Analysis of Turbulent Boundary Layers, Academic Press, 1974.

32. Dhawan, S. and Narasimha, R.: Some Properties of Boundary Layer Flow During Transition from Laminar to Turbulent Motion, J. Fluid Mechanics, Vo1. 3, 1958 .

33. Walker, J. D. A., and Werle, M. J.: Summary and Critique of a Turbulence Model for Freestream Turbulence Effects on Boundary Layer Characteristics, UTRC Report 82-18, 1982.

34. LeBalleur, J. C.: Couplage Visqueux-Non Visquex: Methode Numerique et Applications Aux Ecoulements Bidimensionnels Transsonique Set Supersoniques, La Recherche Aerospatiale, No. 1978-2, pp. 65-76, 1978 .

35. Jansen, B. J. and Mueller, T. J.: Experimental Studies of the Boundary Layer on an Airfoil at Low Reynolds Numbers, AIAA Paper 83-1671, July 1983.

36. Van Dyke, M.: An Album of Fluid Motion, Parabolic Press, Stanford, $\mathrm{CA}, 1982$.

37. Blair, M. F. and Werle, M. J.: The Influence of Free-Stream Turbulence on the Zero Pressure Gradient Fully Turbulent Boundary Layer, AFOSR-81-0514, U.S. Air Force, Sept. 1980.

38. Edwards, D. E., Carter, J. E. and Werle, M. J.: Analysis of the Boundary Layer Equations Including a New Composite Coordinate Transformation--The ABLE Code, UTRC Report 81-30, May 1982.

39. Stewartson, K., and Williams, P. G.: Self-Induced Separation, Proc. Roy. Soc. London, Series A, Vol. 312, No. 1509, September 2, 1969 , pp. 181-206. 


\section{Analysis of the Inviscid Flow Over an Airfoil with a Small Protrusion}

In this appendix the method of matched asymptotic expansions is used to analyze the inviscid flow over an airfoil on which a small protrusion occurs. Figure A-1 shows a schematic diagram of the airfoil with the protrusion details enlarged so that the length scales which characterize this bump can be clearly shown. First, a general formulation of the overall inviscid flow will be presented followed by a detailed description of the outer flow over the airfoil and inner flow in the immediate vicinity of the protrusion. Matching of these two flows through second order will be discussed with the end result being a mathematical formulation of the lead order perturbed inviscid flow near the protrusion which is used in the main text in the local inviscid-viscous interaction analysis. The present analysis is conducted only for incompressible flow; however, the inclusion of compressibility effects should straightforwardly follow the formal approach taken here.

The incompressible, irrotational flow over an airfoil can be described by the full potential equation

$$
\left(\frac{1}{H} \Phi_{\xi}\right)_{\xi}+\left(H \Phi_{\eta}\right)_{\eta}=0
$$

where $\xi$ and $n$ are orthogonal curvilinear coordinates oriented to the smooth airfoil surface as shown in Fig. A-l. The subscripts $\xi$ and $\eta$ denote differentiation in the respective directions. For convenience the origin of the $\xi, n$-coordinate system has been chosen at the center of the protrusion as shown in Fig. A-1. The metric coefficient $H$ is defined as $H=1+k \eta$ where $K$ is the curvature of the airfoil surface (without the protrusion). The tangential and normal velocity components, $U$ and $V$, respectively are related to the velocity potential $\Phi$ by

$$
U=\frac{1}{H} \Phi_{\xi} \quad V=\Phi_{\eta}
$$

The surface boundary condition is given by

$$
\frac{V}{U}=\frac{1}{H} \eta_{0}^{\prime}(\xi)
$$

which is imposed at $n_{0}=\delta T(\xi)$ and requires the flow to be tangent to the airfoil, including the protrusion. Equation (A-3) can also be expressed in terms of the velocity potential which results in

$$
\Phi_{\eta}=\frac{1}{H^{2}} \eta_{0}^{\prime}(\xi) \Phi_{\xi} \text { of } \eta=\eta_{0}
$$


In the present analysis, it is assumed that the streamwise length of the protrusion is $\varepsilon$ where $\varepsilon$ is asymptotically small compared to the chord of the airfoil. Hence, it is seen that over most of the airfoil the usual Neumann condition, $\Phi_{n}(\xi, 0)=0$, is the required boundary condition. In the far field, the flow is required to return to uniform flow which is stated by

$$
\left.\begin{array}{l}
\Phi_{\xi} \rightarrow H \cos \theta \\
\Phi_{\eta} \rightarrow-\sin \theta
\end{array}\right\} \text { os } \xi, \eta \longrightarrow \infty
$$

where $\theta$ is the airfoil surface angle measured with respect to a reference axis.

It is convenient to break the analysis into an outer or global description of the flow, and an inner or local description which is applicable in the immediate vicinity of the protrusion. The relative length scales of the bump are chosen also as to be representative of those of viscous displacement thickness induced by a transitional separation bubble. In our previous investigation (Ref. 21) it was observed that the height/length ratio of the displacement surface produced by a transitional separation bubble was always very small. Therefore, in the present study it is assumed that

$$
\lim _{\epsilon \rightarrow 0} \frac{\delta}{\epsilon}=0
$$

and hence, the protrusion height, $\delta$, is taken to be asymptotically small compared to its length, $\varepsilon$, in the limit of a vanishing protrusion. We now proceed to establish the outer and inner region expansions in terms of the protrusion length, $\varepsilon$.

\section{Outer Region}

The outer potential is expanded in terms of the gauge functions $\Delta_{\mathfrak{n}}$ to give

$$
\Phi^{0}(\xi, \eta ; \epsilon)=\Delta_{1}(\epsilon) \Phi_{1}^{0}(\xi, \eta)+\Delta_{2}(\epsilon) \Phi_{2}^{0}(\xi, \eta)+\ldots
$$

where each of the $\Phi_{\mathrm{n}}^{0}$ are assumed to be $0(1)$. Substitution of $\mathrm{Eq}$. (A-7) into Eq. (A-1) and taking the limit as $\varepsilon \rightarrow 0$ it is seen that each of the $\Phi_{\mathrm{n}}^{\circ}$ satisfies the original partial differential equation.

$$
\left(\frac{1}{H} \Phi_{n \xi}^{o}\right)_{\xi}+\left(H \Phi_{n \eta}^{o}\right)_{\eta}=0
$$

The curvature of the airfoil, $k$, is assumed to be $0(1)$, that is, as $\varepsilon \rightarrow 0$ the airfoil curvature remains finite. 
We now substitute the expansion for the outer potential into the surface boundary condition given by $\mathrm{Eq}$. $(\mathrm{A}-4)$. Since we are assuming the protrusion is thin, that is, its height is small compared to its length, the boundary condition can be transferred via Taylor series from the protrusion surface $n=\eta_{0}$ to $n=0$, the original airfoil surface. The surface boundary condition now becomes

$$
\begin{aligned}
& \Phi_{1 \eta}^{0}(\xi, 0)-\frac{\delta}{\epsilon} T^{\prime}\left[\Phi_{1 \xi}^{0}(\xi, 0)-\frac{\Delta_{2}}{\Delta_{1}} \Phi_{2 \xi}^{0}(\xi, 0)\right]+\frac{\Delta_{2}}{\Delta_{1}} \Phi_{2 \eta}^{0} \\
& +\delta T\left[\Phi_{1 \eta \eta}^{0}(\xi, 0)+\frac{\Delta_{2}}{\Delta_{1}} \Phi_{2 \eta \eta}^{0}(\xi, 0)\right] \\
& +\frac{\delta^{2}}{\epsilon} T T^{\prime}\left[\Phi_{1 \xi \eta}^{0}(\xi, 0)-2 \kappa \Phi_{1 \xi}^{0}(\xi, 0)\right]+\cdots=0
\end{aligned}
$$

In Eq. (A-9), $T^{\prime}$ denotes $d T / d \zeta$ which is an $O(1)$ quantity with $\zeta$ defined as $\xi / \varepsilon$. Equating $O(I)$ terms to zero yields

$$
\Phi_{1 \eta}^{0}(\xi, 0)=0
$$

which is the usual surface boundary condition for the flow over an airfoil with no protrusion.

Substitution of the outer expansion in $\mathrm{Eq} .(\mathrm{A}-7)$ into the far field boundary condition yields

$$
\begin{aligned}
& \Phi_{1 \xi}^{0}+\frac{\Delta_{2}}{\Delta_{1}} \Phi_{2 \xi}^{0}+\cdots \cdot=\frac{H \cos \theta}{\Delta_{1}} \\
& \left.\Phi_{i \eta}^{0}+\frac{\Delta_{2}}{\Delta_{1}} \Phi_{i \eta}^{0}+\cdots=-\frac{\sin \theta}{\Delta_{1}}\right\} \text { as } \xi, \eta \rightarrow \infty
\end{aligned}
$$

As $\varepsilon \rightarrow 0$, it is concluded that $\Delta_{1}$ must remain finite and hence, $\Delta_{1}$, is set to unity for convenience. At this point, the description of the first order outer problem is complete and it is seen that it is simply the flow over the airfoil without a protrusion.

\section{Inner Region}

In the inner region, it is convenient to define stretched coordinates

$$
X=\frac{\xi}{\epsilon} Y=\frac{\eta}{\epsilon}
$$


so that in these coordinates the protrusion length is $0(1)$ and the height is $0(\delta / \varepsilon)$. Equation $(A-6)$ shows that even in these coordinates the protrusion appears as a small disturbance to the local airfoil flow.

The potential function for the inner region is expanded in terms of $\varepsilon$ as

$$
\Phi^{i}\left(\xi, \eta_{i} \epsilon\right)=\delta_{1}(\epsilon) \phi_{1}^{i}(X, Y)+\delta_{2}(\epsilon) \phi_{2}^{i}(X, Y)+\cdots
$$

Substitution of this expansion into the governing equation (A-1) yields

$$
\begin{aligned}
\phi_{1 X X}^{i} & +\phi_{I Y Y}^{i}+\frac{\delta_{2}}{\delta_{1}}\left(\phi_{2 X X}^{i}+\phi_{2 Y Y}^{i}\right)-\epsilon\left(\kappa Y \phi_{I X}^{i}\right)_{X} \\
& +\epsilon\left(\kappa Y \phi_{Y Y}^{i}\right)_{Y}-\frac{\delta_{2} \epsilon}{\delta_{I}}\left[\left(\kappa Y \phi_{2 X}^{i}\right)_{X}-\left(\kappa Y \phi_{2 Y}^{i}\right)_{Y}\right]+\cdots=0
\end{aligned}
$$

Equation (A-14) shows that in the limit of $\varepsilon \rightarrow 0$ the first order inner potential is governed by Laplace's equation

$$
\phi_{i x x}^{i}+\phi_{i Y Y}^{i}=0
$$

The surface boundary condition, Eq. A-4, is expressed in terms of the inner region potential as

$$
\phi_{\eta}^{i}=\frac{1}{H^{2}} \eta_{0}^{\prime} \phi_{\xi}^{i}
$$

which is implemented at $\mathrm{Y}=\mathrm{Y}_{0}=\delta / \varepsilon \mathrm{T}(\mathrm{X})$ where $\mathrm{T}(\mathrm{X})$ is an $0(1)$ function. Since the protrusion is treated as asymptotically thin, even in inner coordinates the surface boundary condition can be transferred to $\mathrm{Y}=0$ with a Taylor series expansion. Substitution of the inner expansion for the potential function Eq. (A-13), into the surface boundary condition, Eq. $(A-16)$, and the use of a Taylor series yields the following relation to be satisfied at $Y=0$ :

$\phi_{Y Y}^{i}(x, 0)+\frac{\delta_{2}}{\delta_{1}} \phi_{2 Y}^{i}(x, 0)+\frac{\delta}{\epsilon}\left[T_{\phi_{Y Y}}^{i}(x, 0)-T^{\prime} \phi_{i X}^{i}(x, 0)\right]$

$+\frac{\delta_{2}}{\delta_{1}} \frac{\delta}{\epsilon}\left[T \phi_{2 Y Y}^{i}(x, 0)-T^{\prime} \phi_{2 X}^{i}(x, 0)\right]-\left(\frac{\delta}{\epsilon}\right)^{2} T T^{\prime} \phi_{i X X}^{i}(x, 0)$

$$
+2 \delta \kappa Y T^{\prime} \phi_{i x}^{i}(x, 0)+\cdots=0
$$

As $\varepsilon \rightarrow 0$ the $O(1)$ surface boundary condition is

$$
\phi_{I_{Y}}^{\prime}(x, 0)=0
$$


and thus we see that to first order neither the inner or outer solution is affected by the presence of the protrusion for the limit considered here $(\delta / \varepsilon<1)$.

Matching the 1-term inner limit of the outer expansion with the 1-term outer limit of the inner expansion yields

$$
\operatorname{Cx}_{1 \mathrm{x}}^{0}(0,0)=\delta_{1} \phi_{i}(\infty, \infty)
$$

In order for these solutions to match we set $\delta_{1}=\varepsilon$. The solution for the inner first order potential function is now established by Eqs. A-15, A-18, and $A-19$ and is simply

$$
\phi_{1}^{i}(X, Y)=u_{1}^{0}(0,0) X
$$

where $U_{1}^{\circ}(0,0)=\Phi_{1 X}^{0}(0,0)$ is the airfoil surface speed evaluated at the protusion location, $\xi=0, \eta=0$, on the airfoil surface. So we see that the influence of the protrusion is relegated to the second order problem which will be discussed next.

\section{Second Order Analysis}

The governing equation for the second order outer problem has already been given as Eq. (A-8) with $n=2$. The surface boundary condition, Eq. $(A-9)$ is written as

$\Phi_{2 \eta}^{0}(\xi, 0)-\frac{\delta}{6 \Delta_{2}} T^{\prime} \Phi_{1 \xi}^{0}(\xi, 0)+\frac{\delta}{\Delta_{2}} T \Phi_{1 \eta \eta}^{0}(\xi, 0)+\ldots=0$

This relation shows that the inner boundary condition on the second order outer potential is determined by the choice of the gauge function, $\Delta_{2}$. If we choose $\Delta_{2}=\delta / \varepsilon$, the height to length ratio of the protrusion, then the inner boundary condition for the second order outer problem will be the usual small disturbance boundary condition given by the first two terms in Eq. $(A-21)$. However, since we want this boundary condition to be met by the inner solution, $\Delta_{2}$ is chosen such that $\Delta_{2}>\delta / \varepsilon$, that is

$$
\lim _{\epsilon \rightarrow 0} \frac{\delta}{\epsilon \Delta_{2}}=0
$$

thereby resulting in

$$
\Phi_{2 \eta}^{0}(\xi, 0)=0
$$

Since the outer boundary condition for the second order outer potential is homogeneous as deduced from Eq. (A-11), then the solution for the second order outer potential which satisfies $\mathrm{Eq} \cdot(\mathrm{A}-23)$ and the governing equation 
is the trivial solution $\Phi_{2}^{0}(\xi, n)=0$. This result is expected since the first order inner solution was found to be unaffected by the presence of the protrusion.

The second order inner problem is now examined and will show for the first time the explicit appearance of the protrusion characteristics. The governing equation, (Eq. $(A-14)$, is rewritten as follows with the first order inner solution inserted:

$$
\phi_{2 X X}^{i}+\phi_{2 Y y}^{i}-\frac{\epsilon^{3}}{\delta_{2}} y \frac{d x}{d \xi} u_{1}^{0}(0,0)+\ldots=0
$$

The gauge function, $\delta_{2}$, is chosen so that $\delta_{2}>\varepsilon^{3}$ which results in

$$
\phi_{2 X x}^{i}+\phi_{2 Y y}^{i}=0
$$

The surface boundary condition, Eq. $(A-17)$ is rewritten as

$$
\begin{aligned}
\phi_{2 Y}^{i}(x, 0) & -\frac{\delta}{\delta 2} T^{\prime} \phi_{1 X}^{i}(x, 0)+\frac{\delta}{\epsilon}\left[\phi_{2 Y Y}^{i}(x, 0)-T^{\prime} \phi_{2 X}^{i}(x, 0)\right], \\
& +\frac{2 \delta \epsilon}{\delta_{2}}\left\lfloor K Y T^{\prime} \phi_{1 X}^{i}(x, 0)\right]+\ldots=0
\end{aligned}
$$

Since we want the inner solution to describe the flow over the protrusion the gauge function, $\delta_{2}$, is set equal to the protrusion height, $\delta$, which results in

$$
\phi_{2 Y}^{i}(x, 0)=U^{0}(0,0) T^{\prime}
$$

Equation (A-27) is the usual small disturbance surface tangency condition which in this case is that of a thin protrusion subjected to the local velocity of the outer solution evaluated at the protrusion origin.

The outer boundary condition on the second order inner potential is determined by matching the inner and outer expansions. The matching condition between the 2-term inner and 2-term outer expansions leads to

$\phi_{2}^{i}(\infty, \infty)=\frac{\epsilon^{2}}{2 \delta}\left[x^{2} \Phi_{1 \xi \xi}^{0}(0,0)+x y \Phi_{i \xi \eta}^{0}(0,0)+y^{2} \Phi_{i \eta \eta}^{0}(0,0)\right](A-28)$

The least restrictive condition that can be imposed at this point is to choose the limit

$$
\lim _{\epsilon \rightarrow 0} \frac{\epsilon^{2}}{8}=0
$$


which results in

$$
\phi_{2}^{i}(\infty, \infty)=0
$$

At this point two-term inner and outer expansions have been derived which describe the local disturbance due to a protrusion placed on the airfoil. For the particular limit where the bump height, $\delta$, relative to the length, $\varepsilon$, satisfies the inequality $\varepsilon^{2}<\delta<\varepsilon$, it has been determined from matched asymptotic expansions that to lowest order the disturbed flow over the protrusion is governed by Laplace's equation with the usual small disturbance boundary condition at the airfoil surface and uniform flow at a large distance from the protrusion. This uniform flow velocity is that of the first order outer solution over the airfoil evaluated at the protrusion origin. It is observed that to second order, the inner solution is independent of the airfoil curvature. Further examination shows that explicit dependence on the airfoil curvature does occur in the third order inner solution. It is deduced from this analysis that to second order the outer solution is unaffected by the presence of the protrusion.

It is of interest to compare the particular protrusion height to length ratio chosen in the present analysis with that inherent in triple deck theory for strongly interacting laminar flows. Triple deck theory is a multi-layer asymptotic analysis developed by Stewartson and Williams (Ref.39) in which the wall layer, of length, $\varepsilon^{3}=\operatorname{Re}^{-3} / 8$ and height, $\varepsilon^{5}=$ $\operatorname{Re}^{-3 / 8}$, plays a role analogous to that of the bump in the present study. Triple deck theory has been successfully used in numerous strongly interacting viscous flow applications to delineate the mathematical structure as the Reynolds number tends to infinity. Even though triple deck theory has been limited thus far to laminar flows and the present flow under study is transitional, nonetheless it is of interest to determine as to whether or not triple deck theory overlaps with the present analysis. In the present work we are assuming that $\delta>\varepsilon^{2}$ which is equivalent to stating that the bump height to length ratio is

$$
\left.\frac{\text { height }}{\text { length }}\right|_{\text {bump }}>\text { length }
$$

In triple deck theory the inner deck dimensions yield

$$
\left.\frac{\text { height }}{\text { length }}\right|_{\substack{\text { triple } \\ \text { deck }}}=1 \text { ength } 2 / 3
$$

Since the length of the inner deck is vanishingly small as $\operatorname{Re} \rightarrow \infty$, it is seen that the triple deck height to length ratio satisfies Eq. $(\mathrm{A}-31)$ and hence, we can state that the particular protrusion dimensions chosen in the present analysis are inclusive of those contained in triple deck theory. 

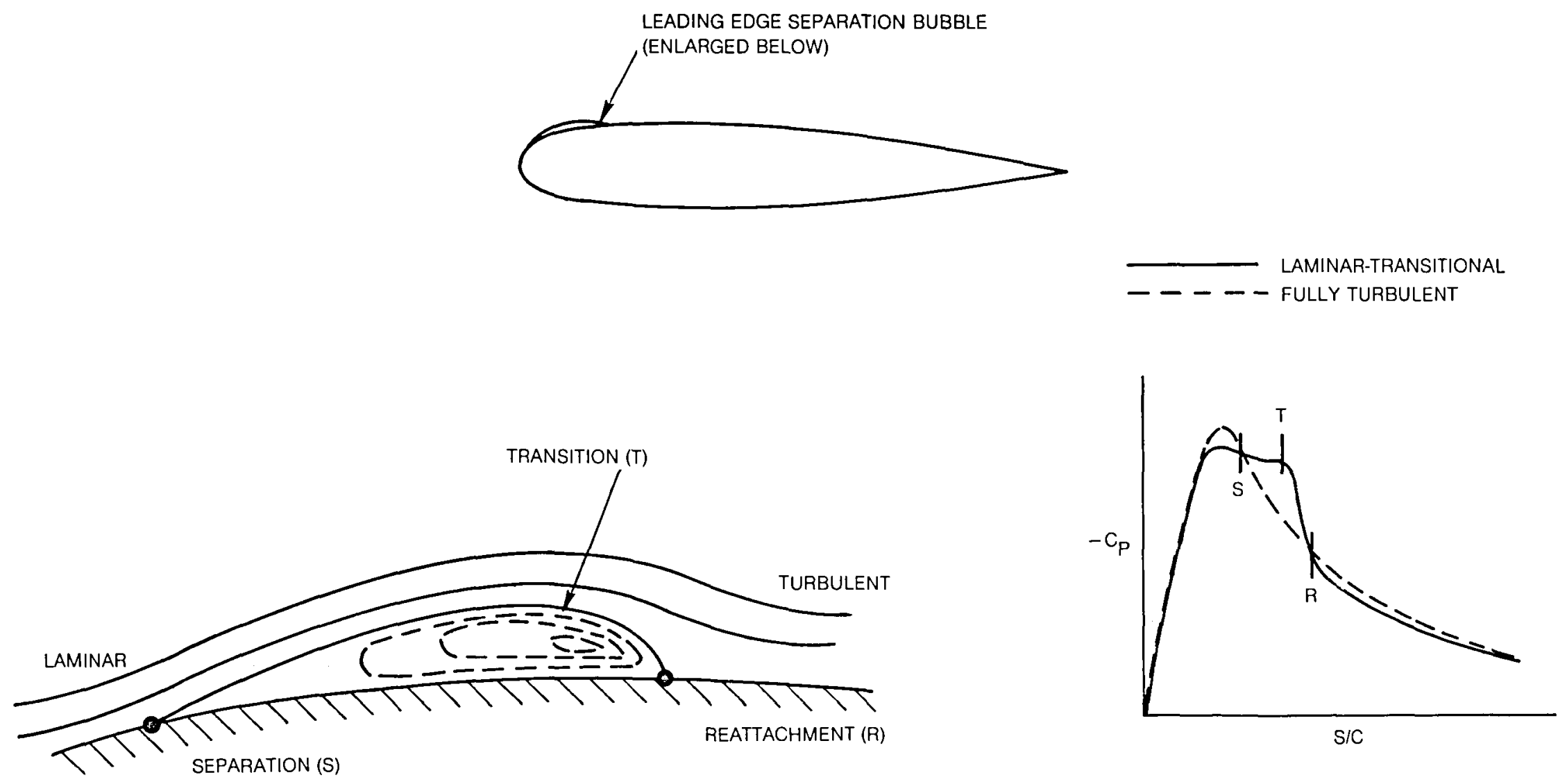

Fig. 1 Schematic diagram of airfoil laminar-transitional separation bubble and pressure distribution 


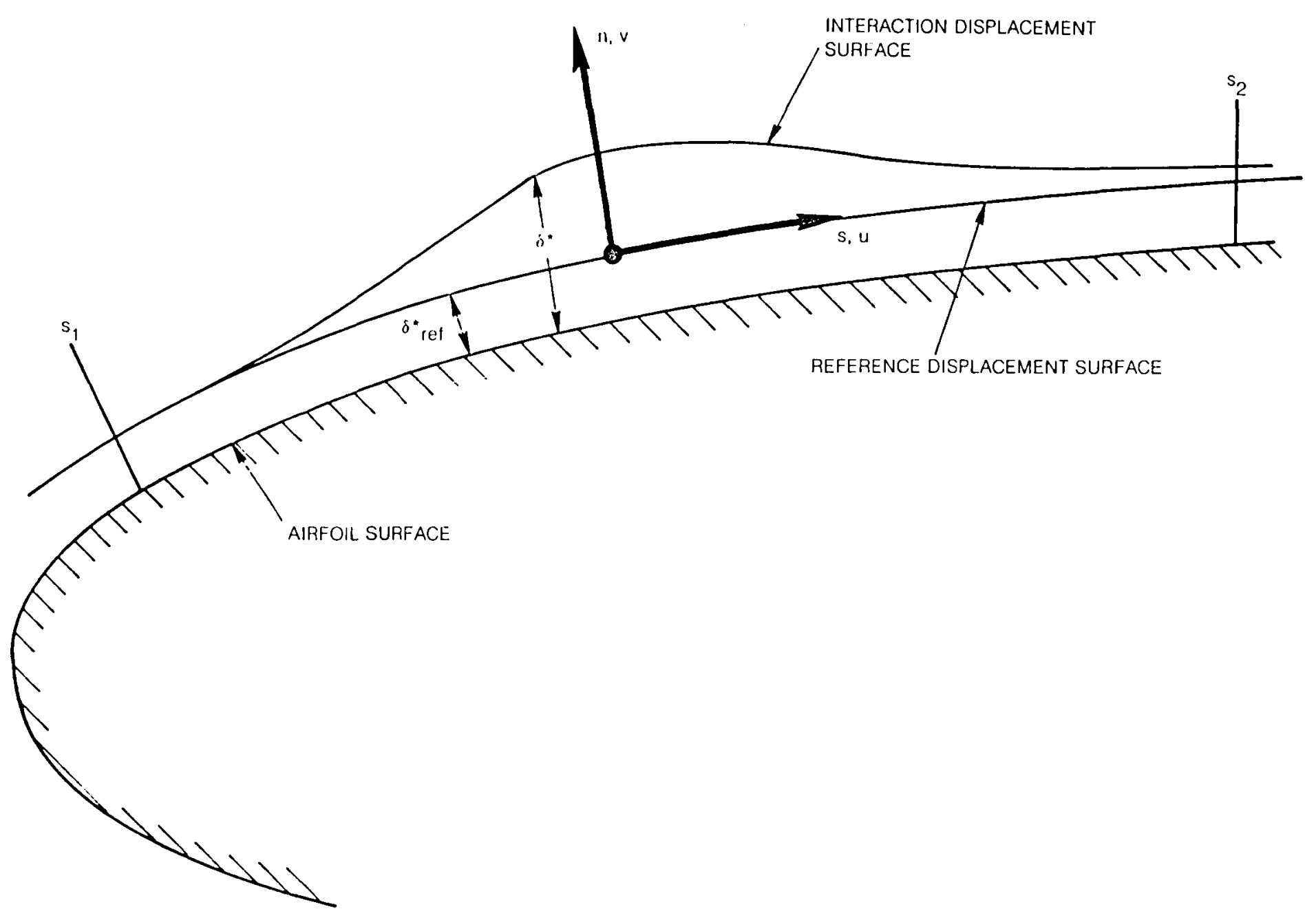

Fig. 2 Local interaction region coordinate system 


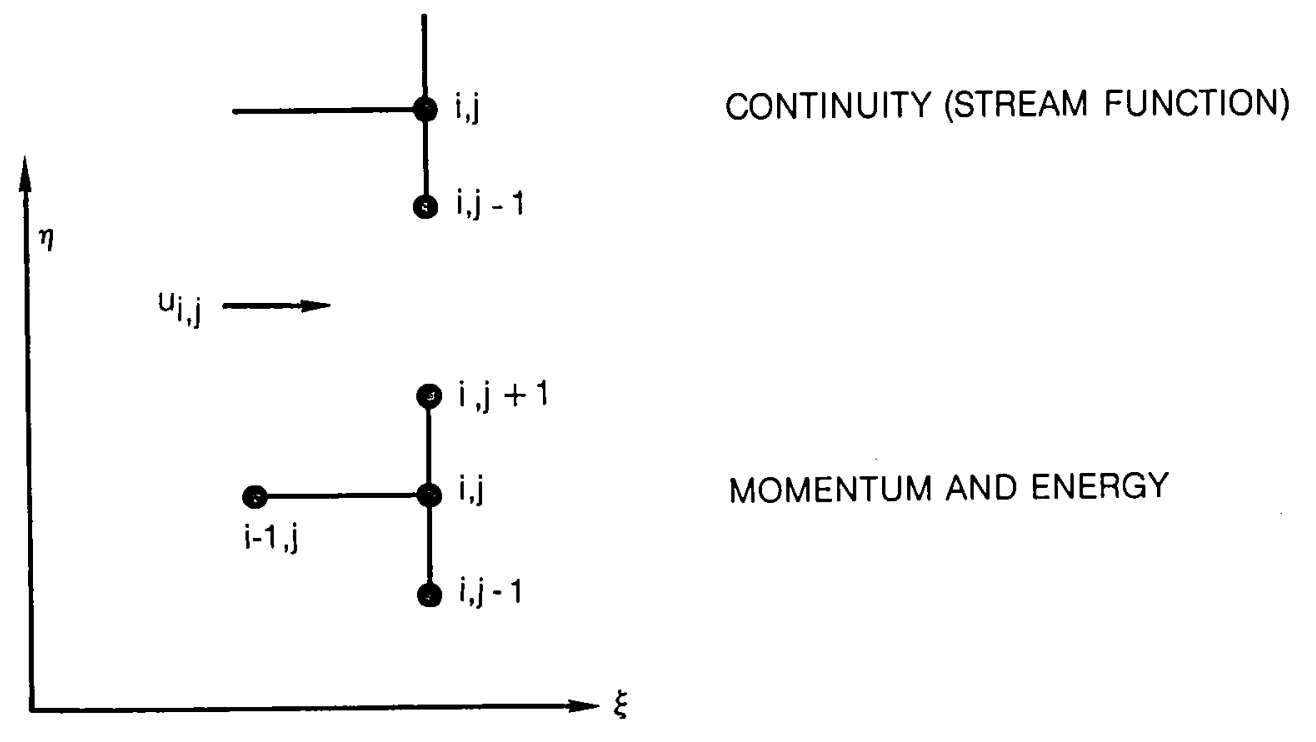

Fig. 3 Finite difference cell structure for the boundary layer equations - attached flow. 


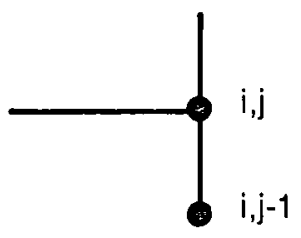

CONTINUITY (STREAM FUNCTION)

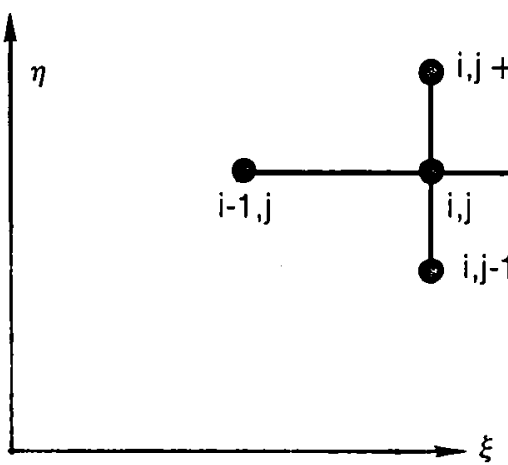

MOMENTUM AND ENERGY

Fig. 4 Finite difference cell structure for boundary layer equations - reversed flow. 


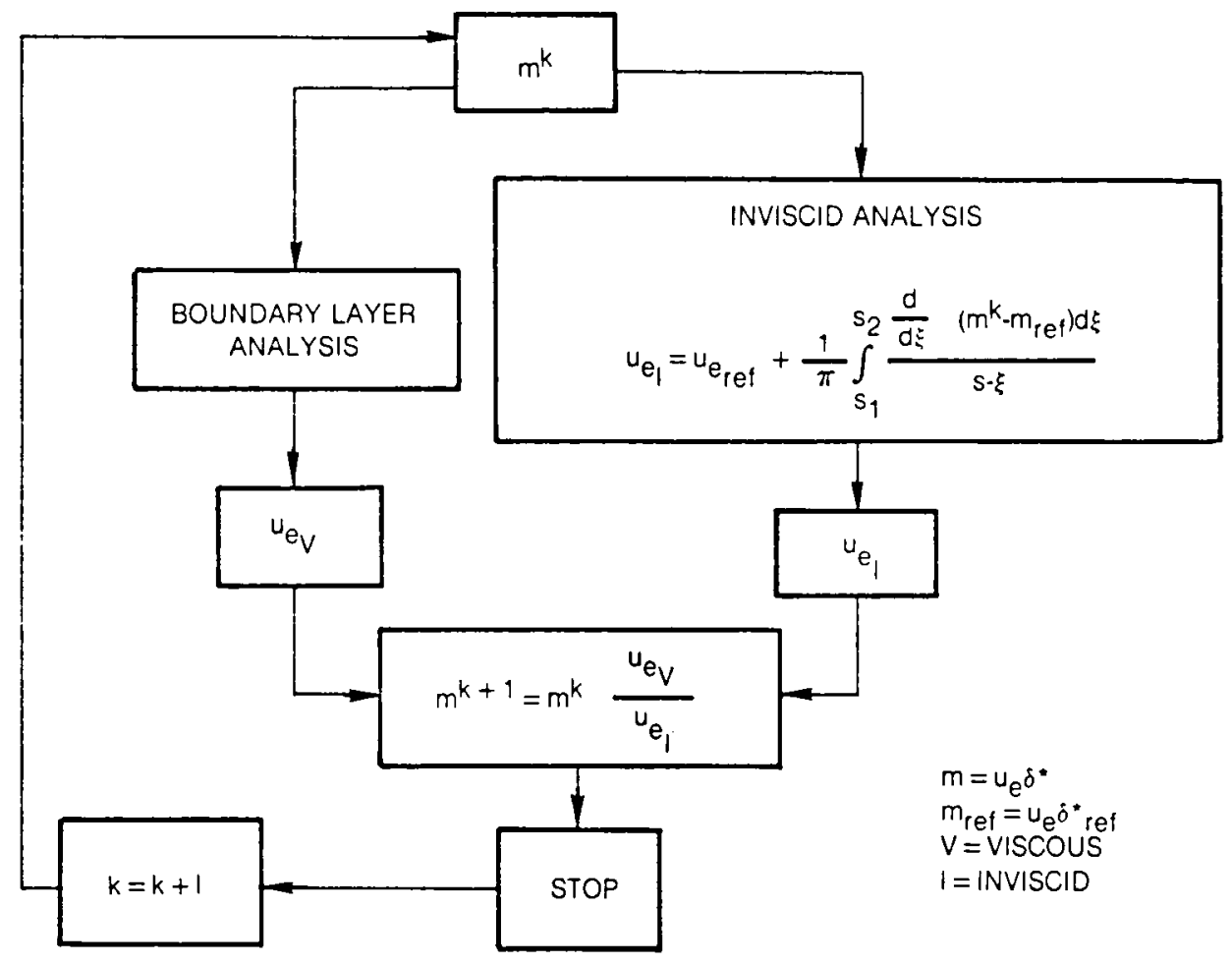

Fig. 5 Inviscid-viscous iteration procedure 


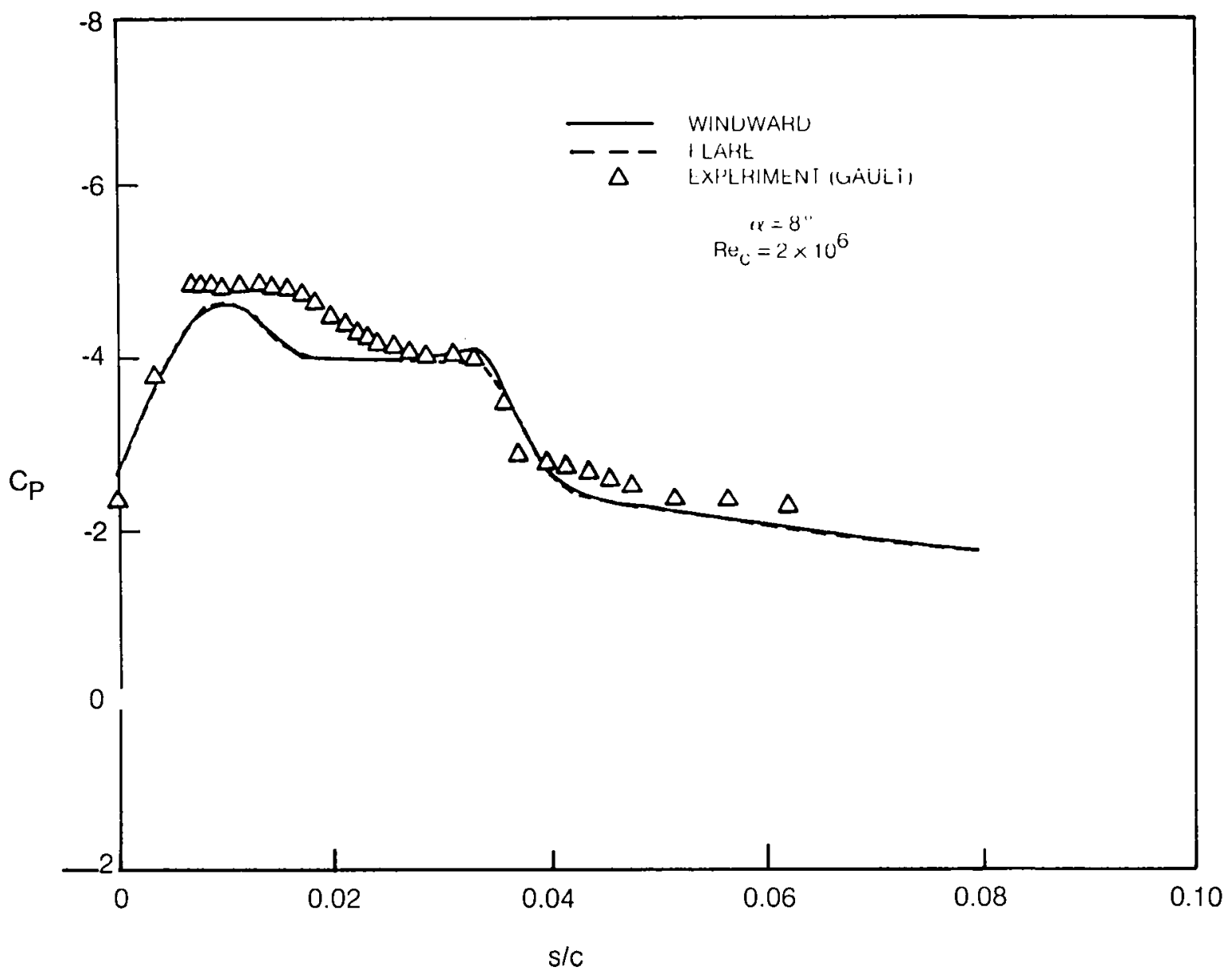

Fig. 6 Comparison of results for windward and FLARE differencing - NACA.0010 airfoil (modified).

(a) Pressure distribution. 


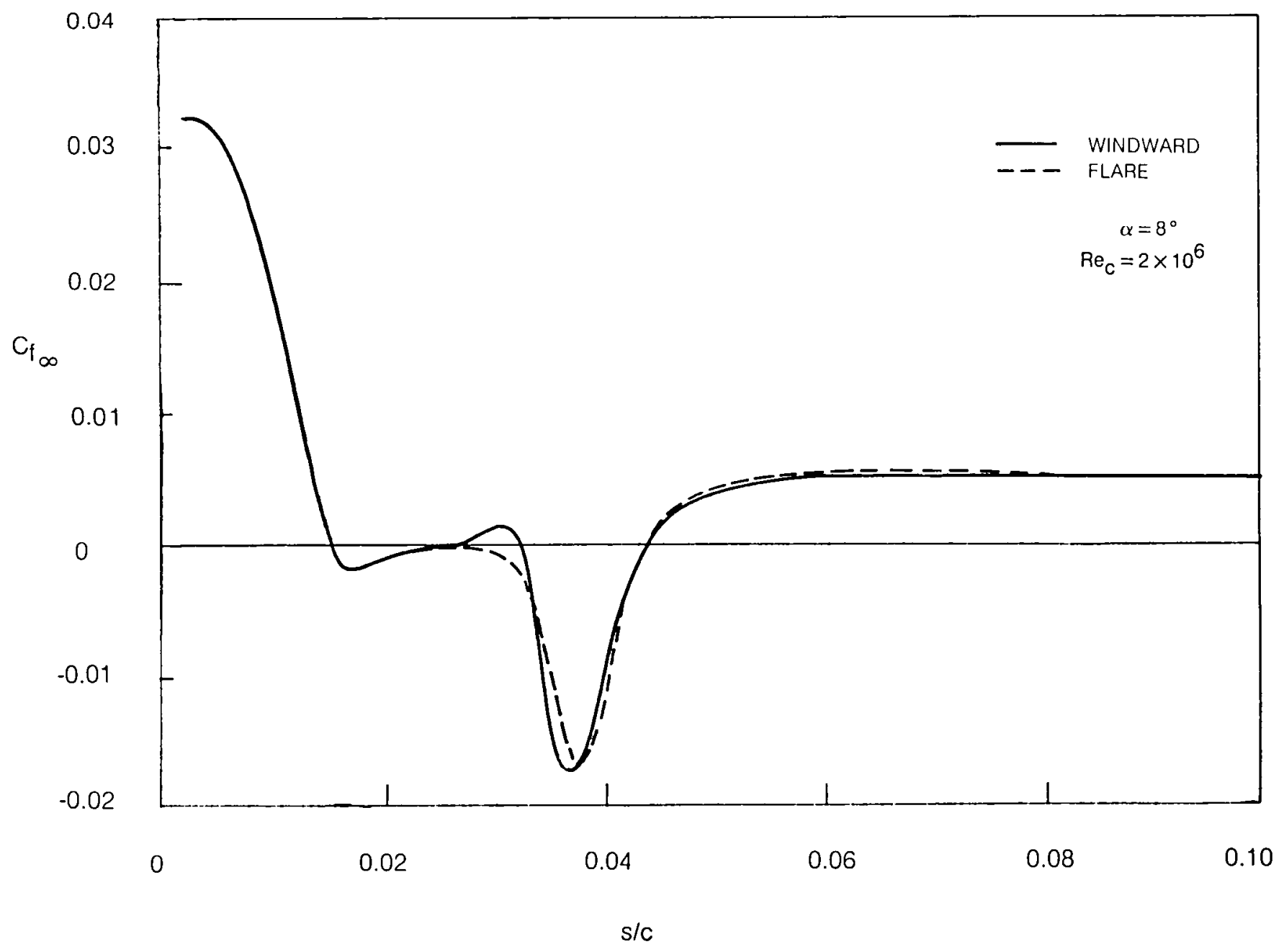

Fig. 6 Comparison of results for windward and FLARE differrencing - NACA.0010 airfoil (modified).

(b) Skin friction 


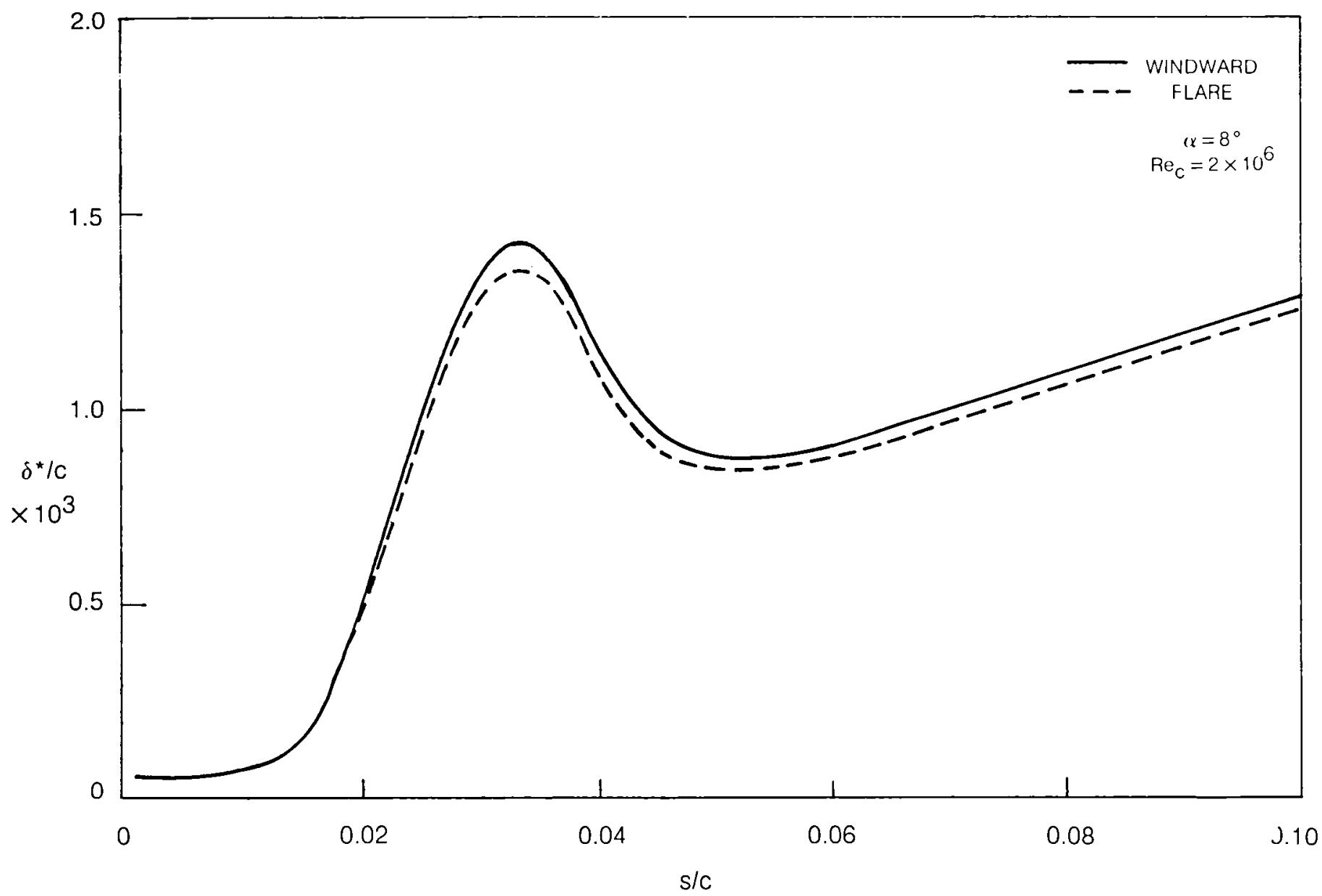

Fig. 6 Comparison of results for windward and FLARE differencing - NACA-0010 airfoil (modified).

(c) Displacement thickness 


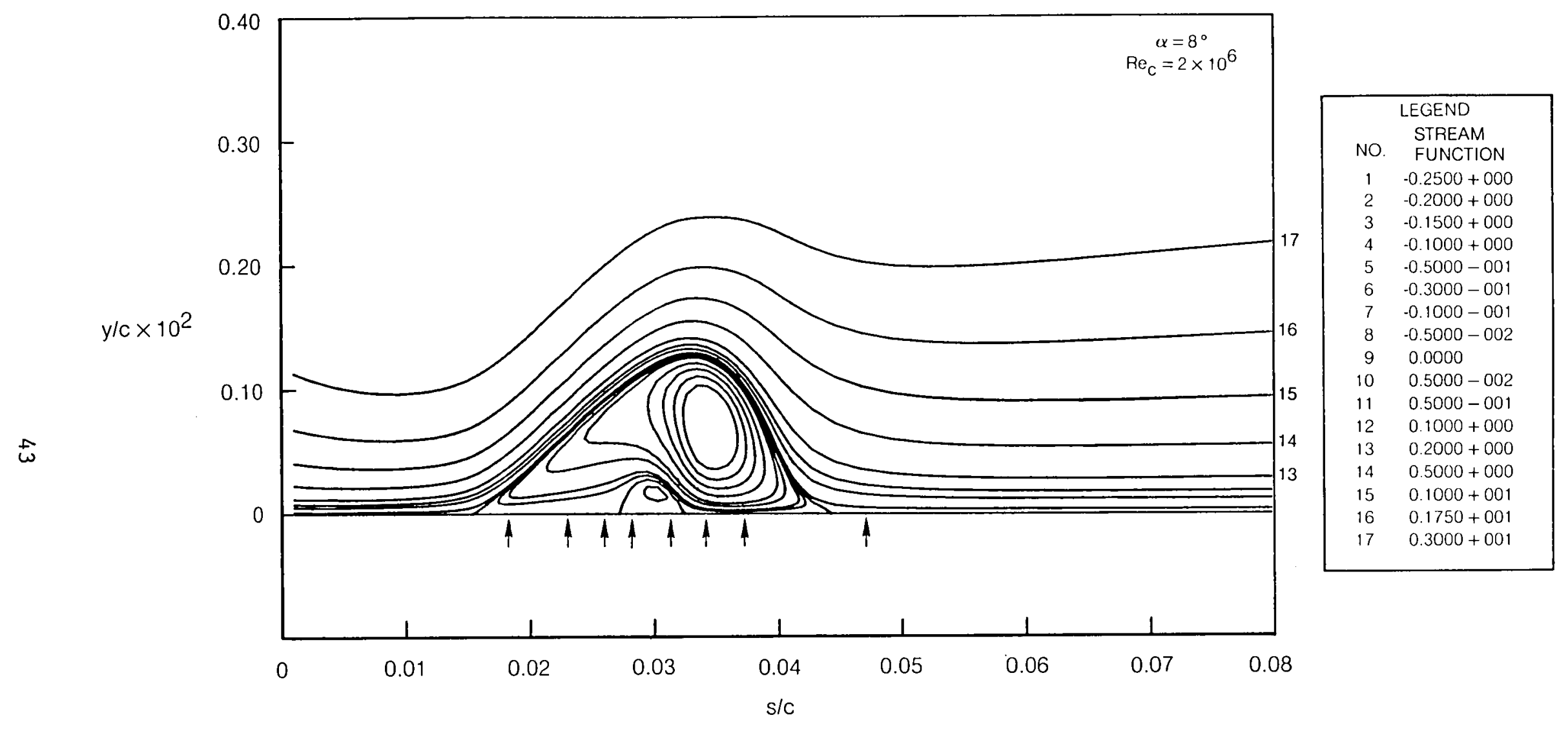

Fig. 7 Transitional separation bubble streamline pattern - NACA.0010 airfoil (modified).

(a) Windward differencing 


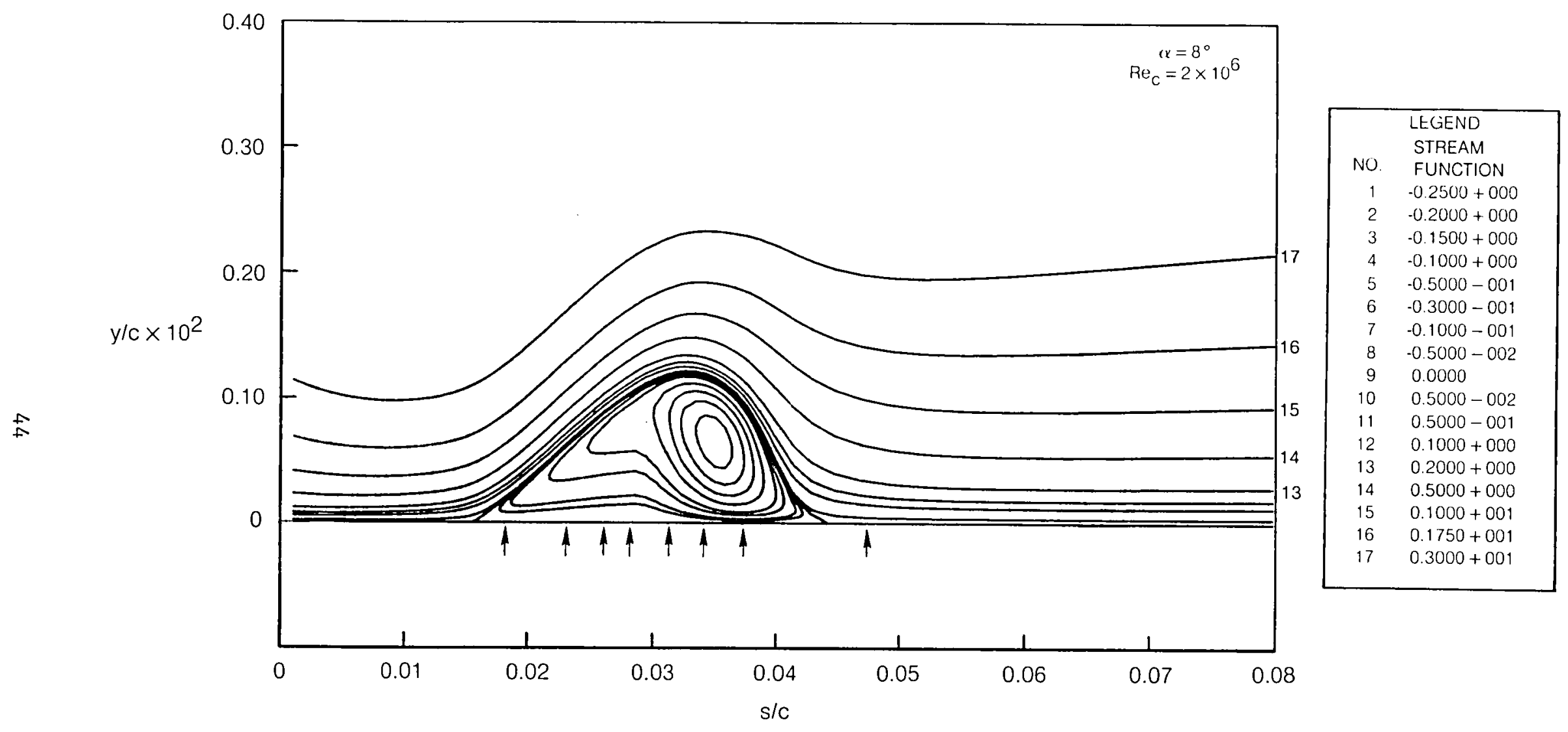

Fig. 7 Transitional separation bubble streamline pattern - NACA.0010 airfoil (modified). (b) FLARE approximation 


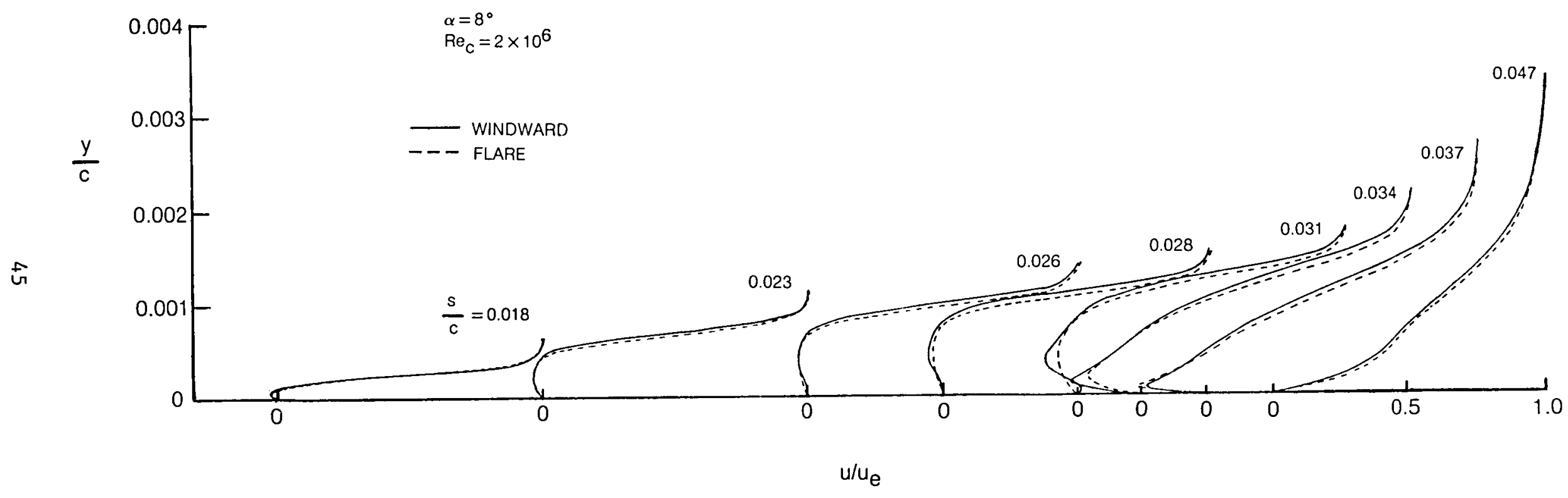

Fig. 8 Velocity profiles from windward and FLARE differencing - NACA.0010 airfoil (modified). 


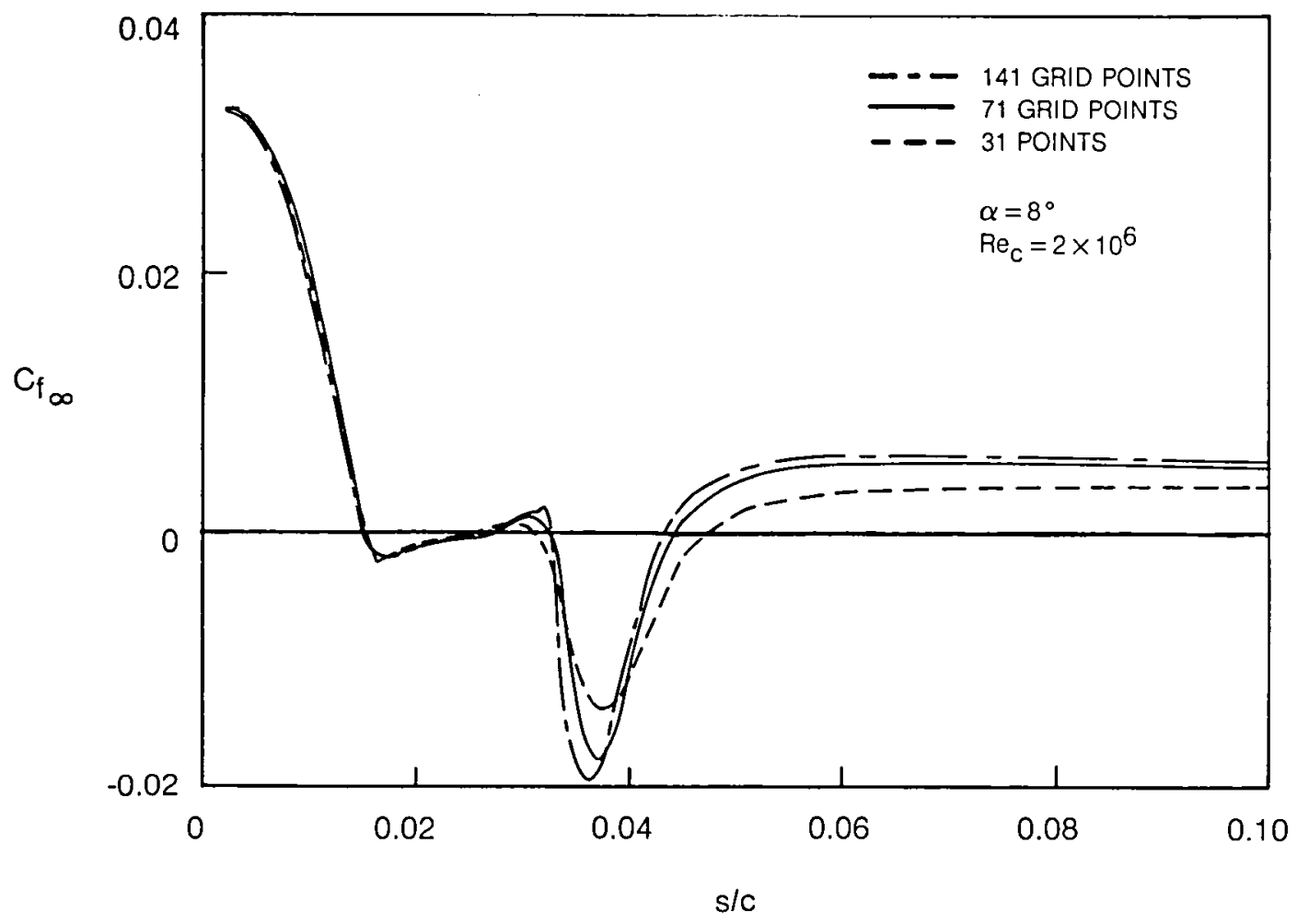

Fig. 9 Effect of streamwise mesh size on skin friction from windward differencing. NACA-0010 airfoil (modified) 


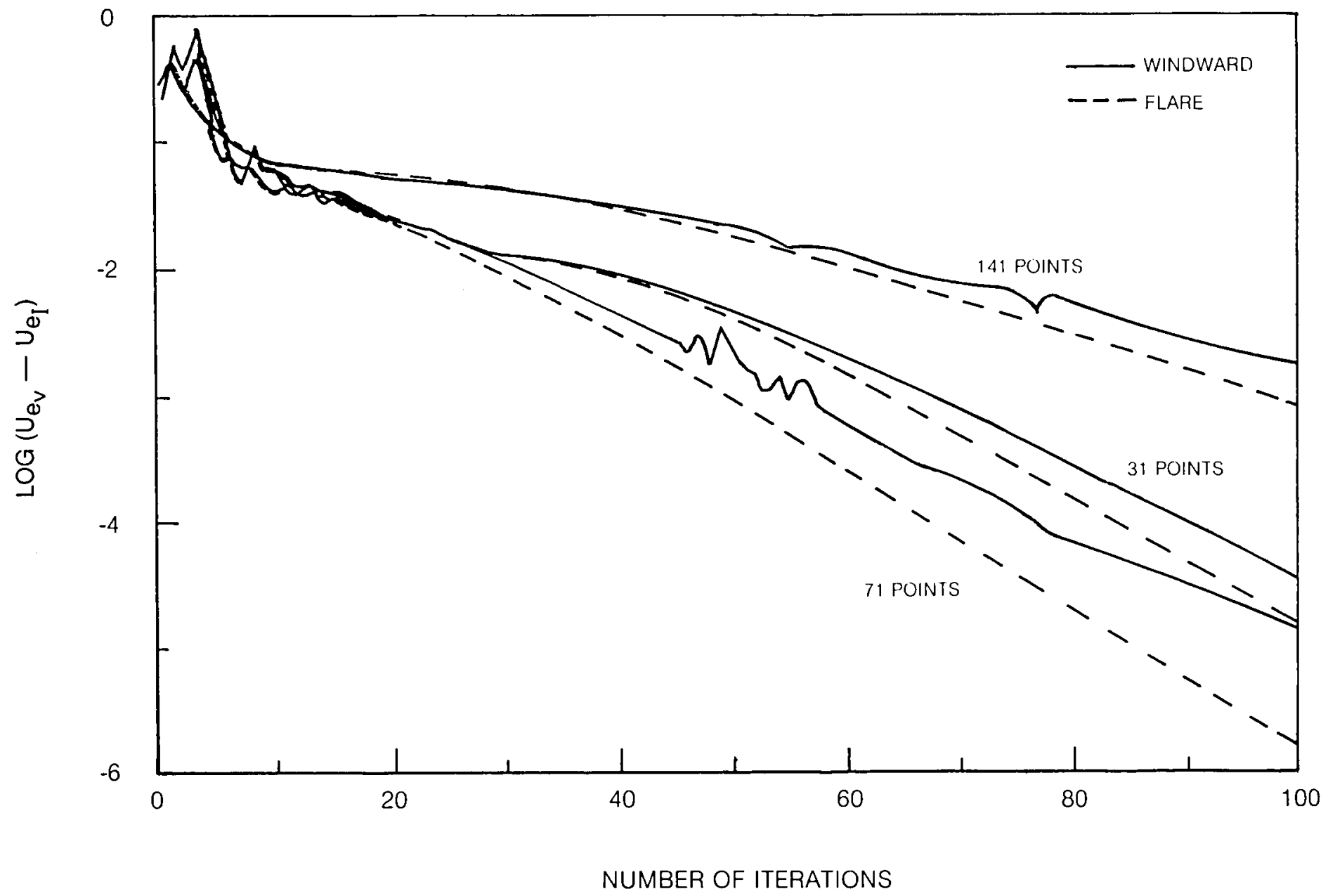

Fig. 10 Global convergence history - NACA-0010 airfoil (modified). 


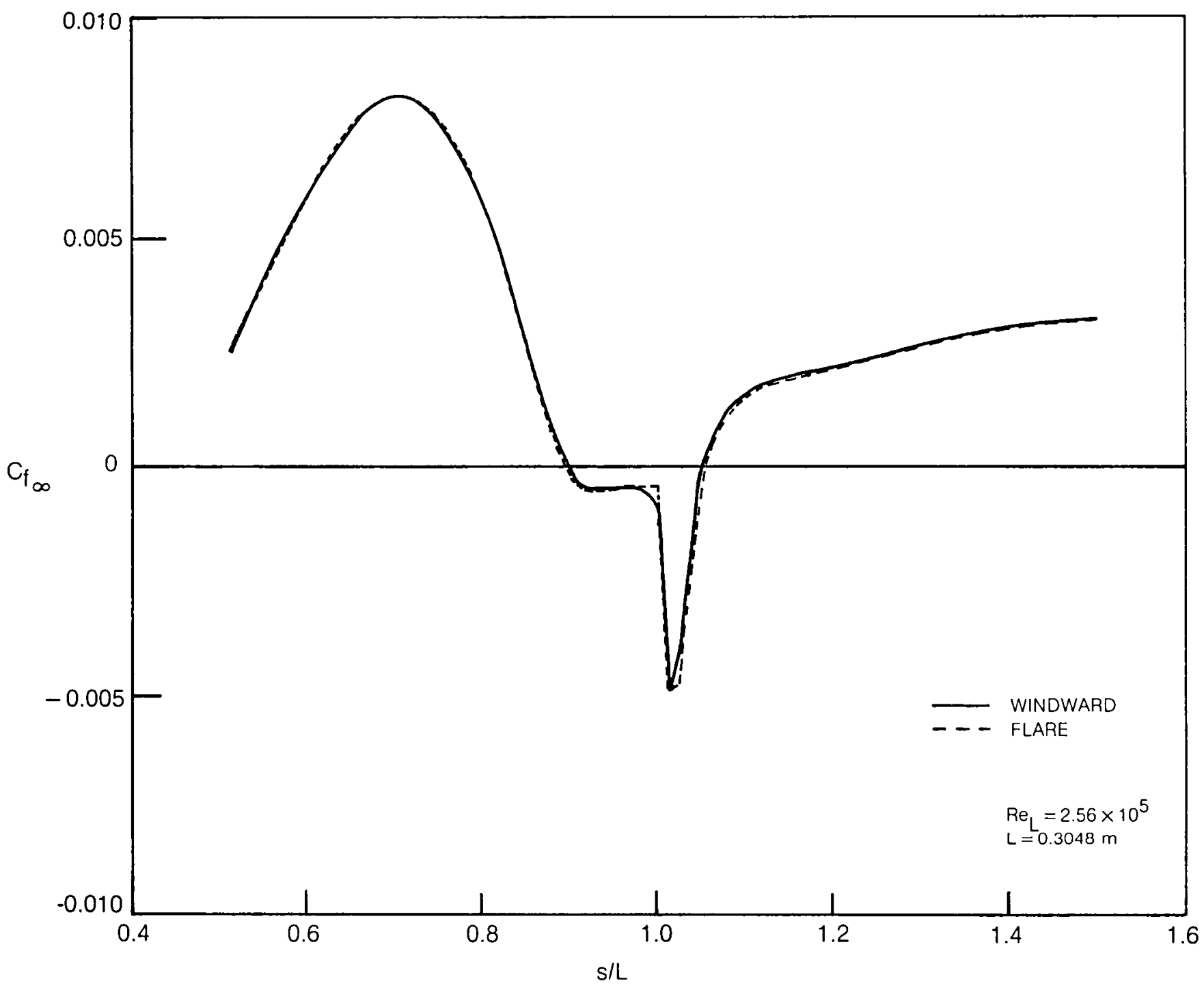

Fig. 11 Comparison of windward and FLARE differencing for the Gaster experiment. (a) Skin friction. 


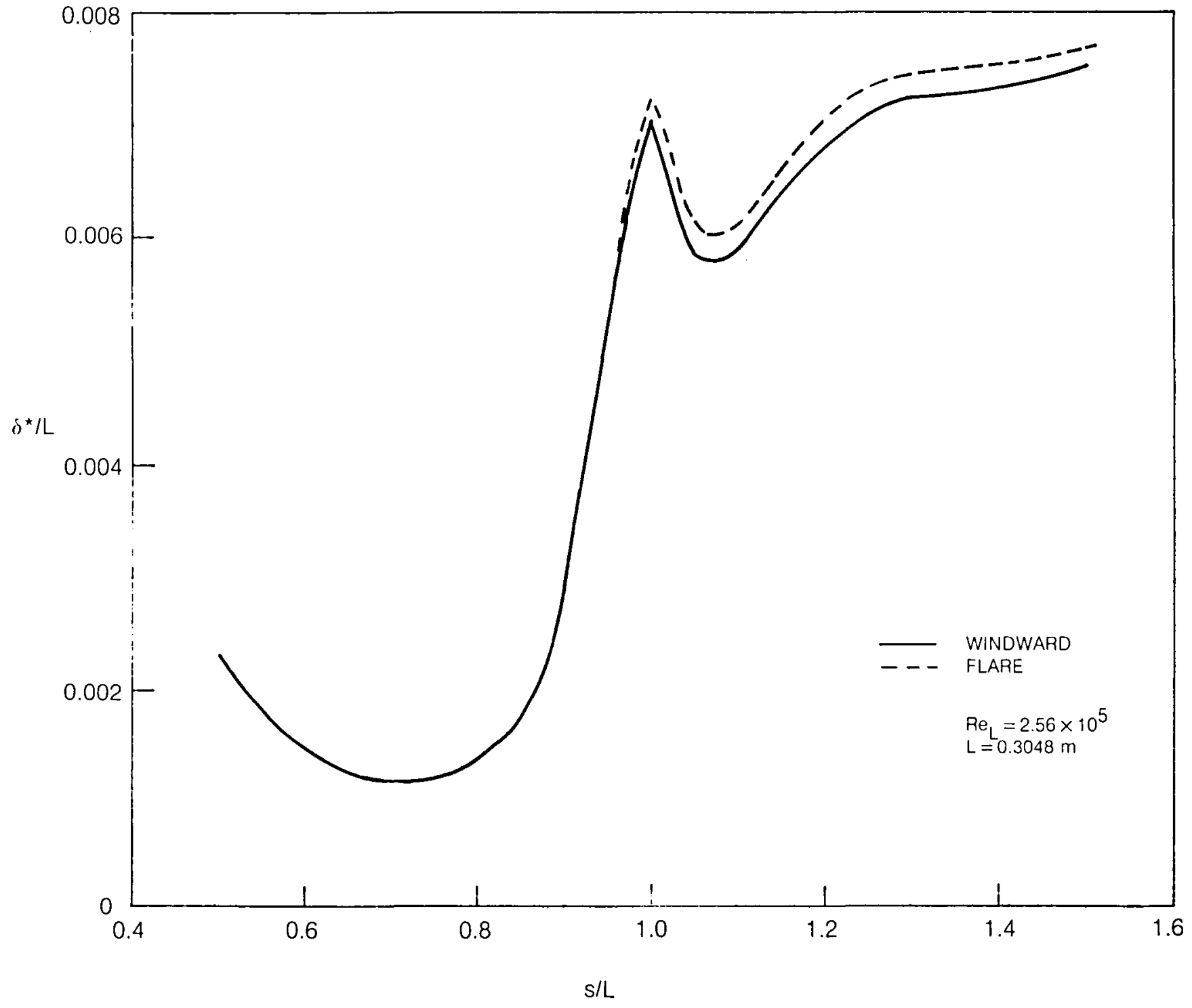

Fig. 11 Comparison of windward and FLARE differencing for the Gaster experiment. (b) Displacement thickness. 


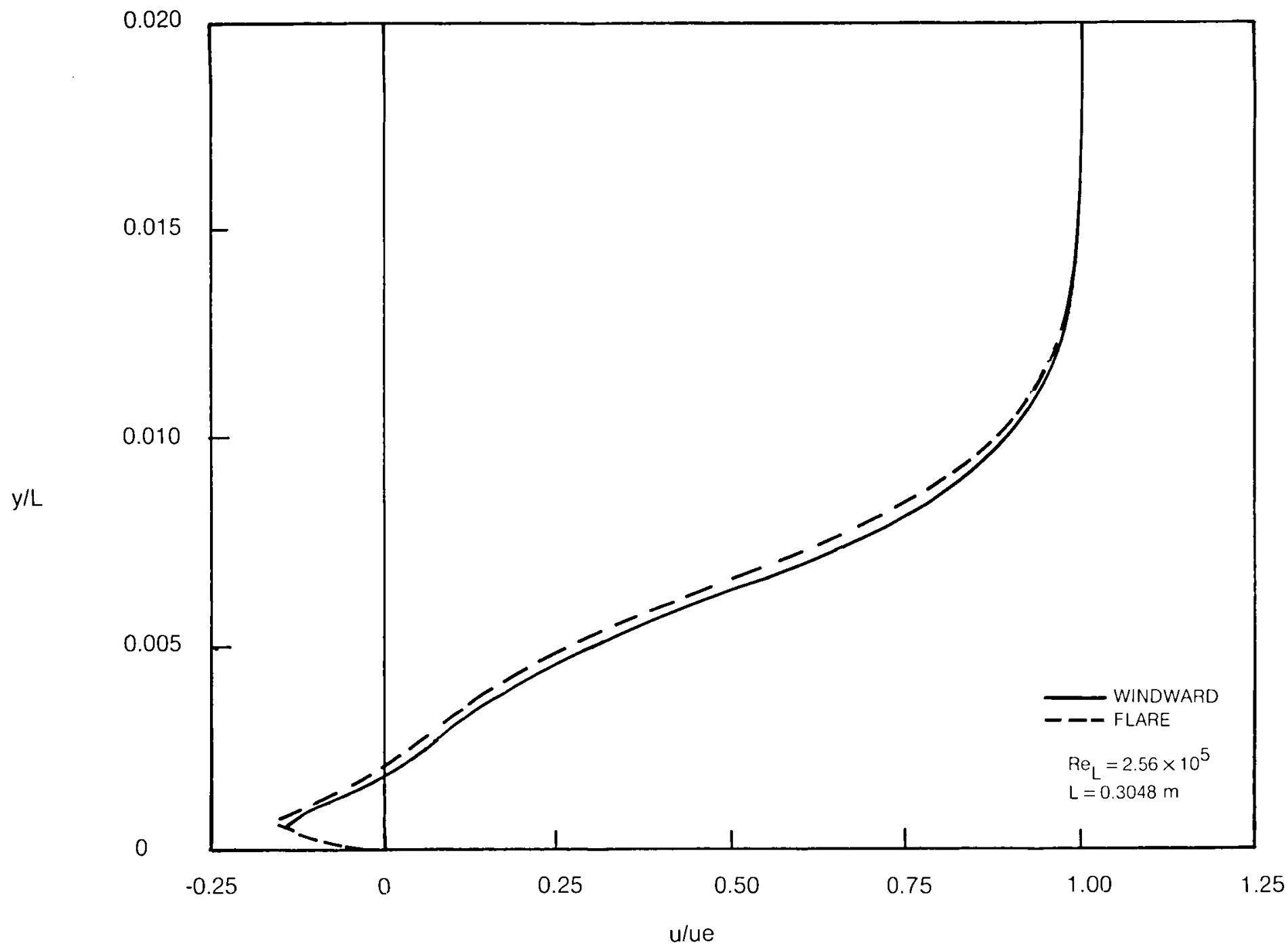

Fig. 12 Comparison of velocity profile at $s / L=1.0125$ for windward and FLARE differencing for Gaster case. 


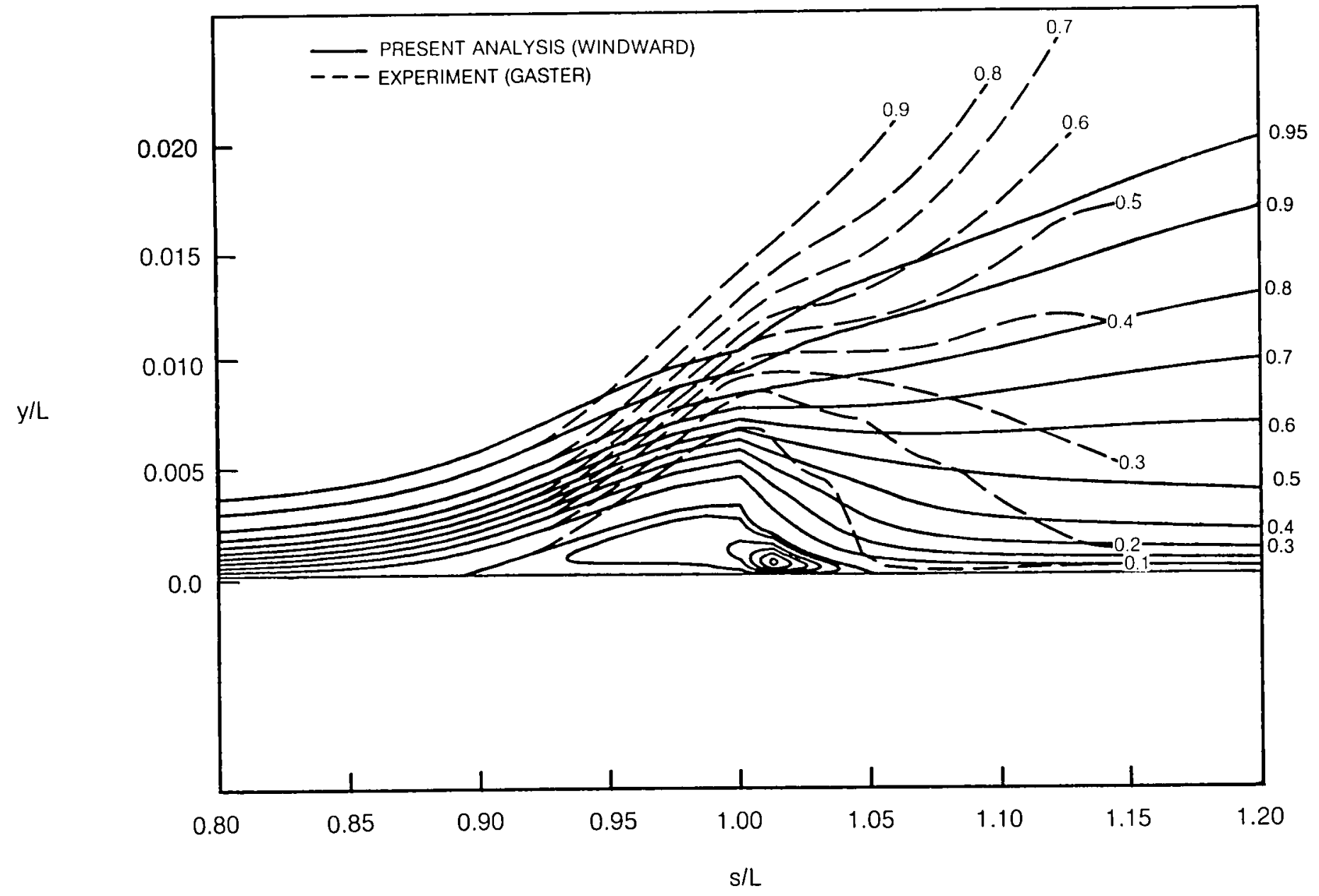

Fig. 13 Iso-velocity contours for Gaster case. 


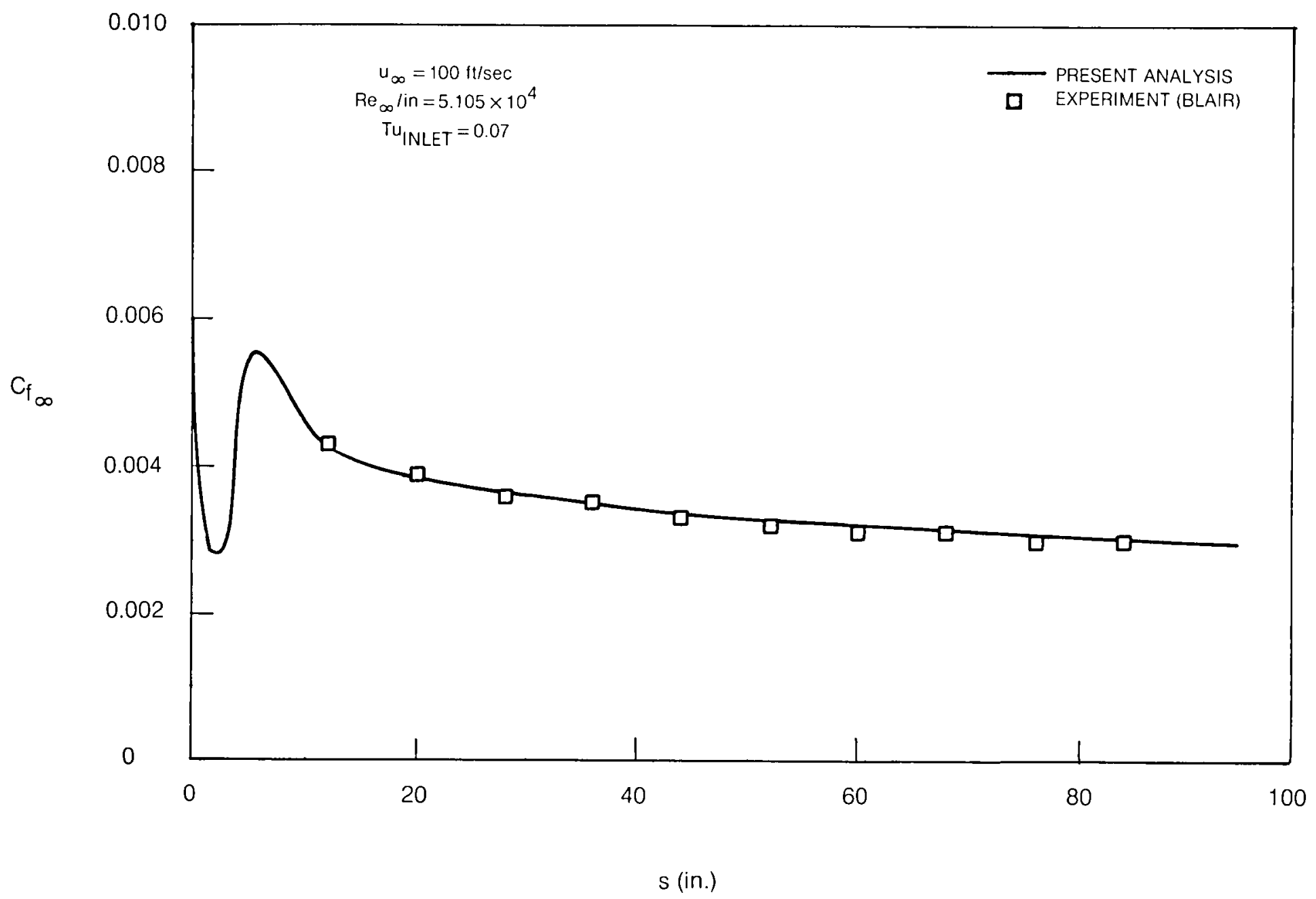

Fig. 14 Flow over flat plate at high free stream turbulence level (a) Skin friction. 


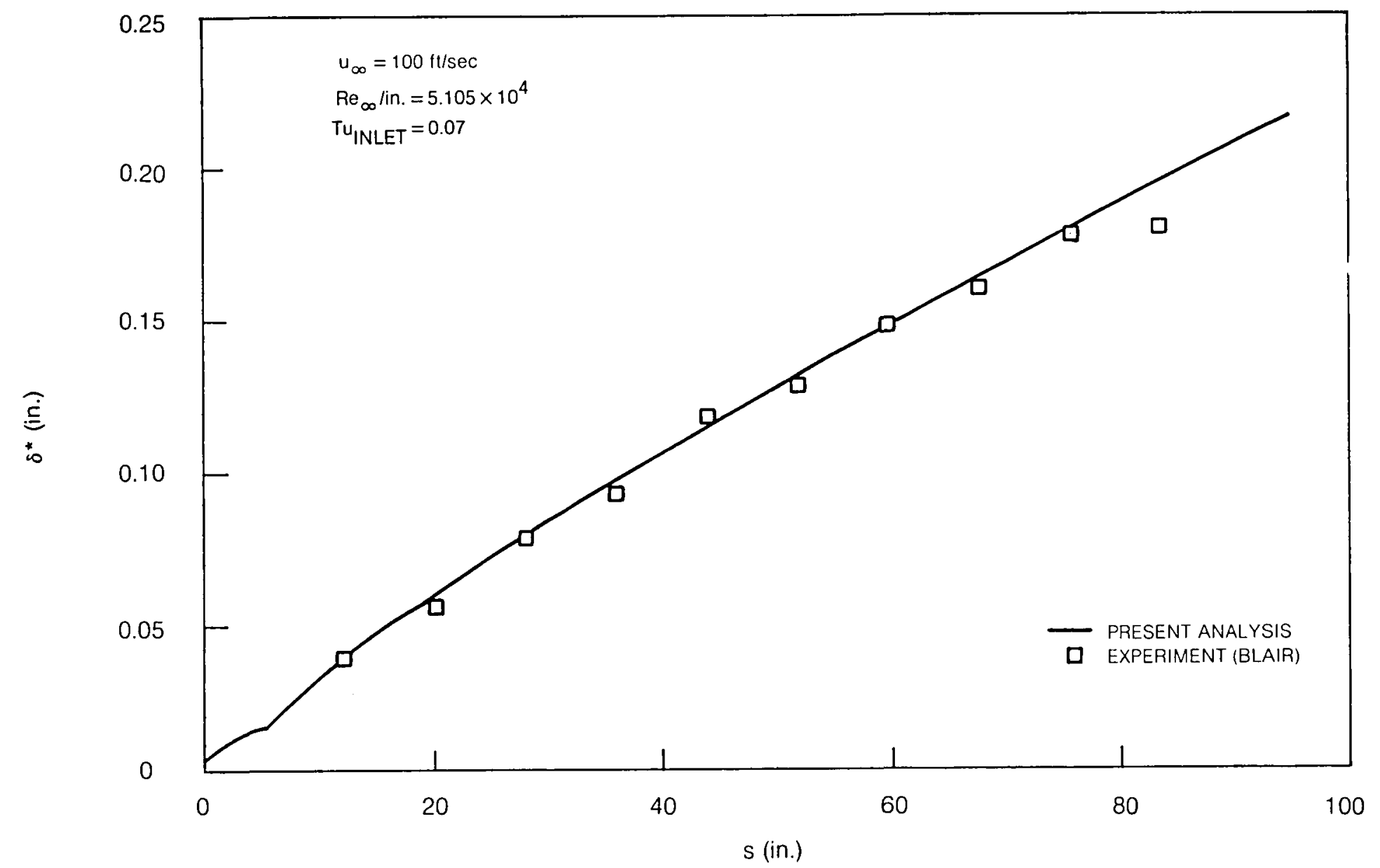

Fig. 14 Flow over flat plate at high free stream turbulence level. (b) Displacement thickness 


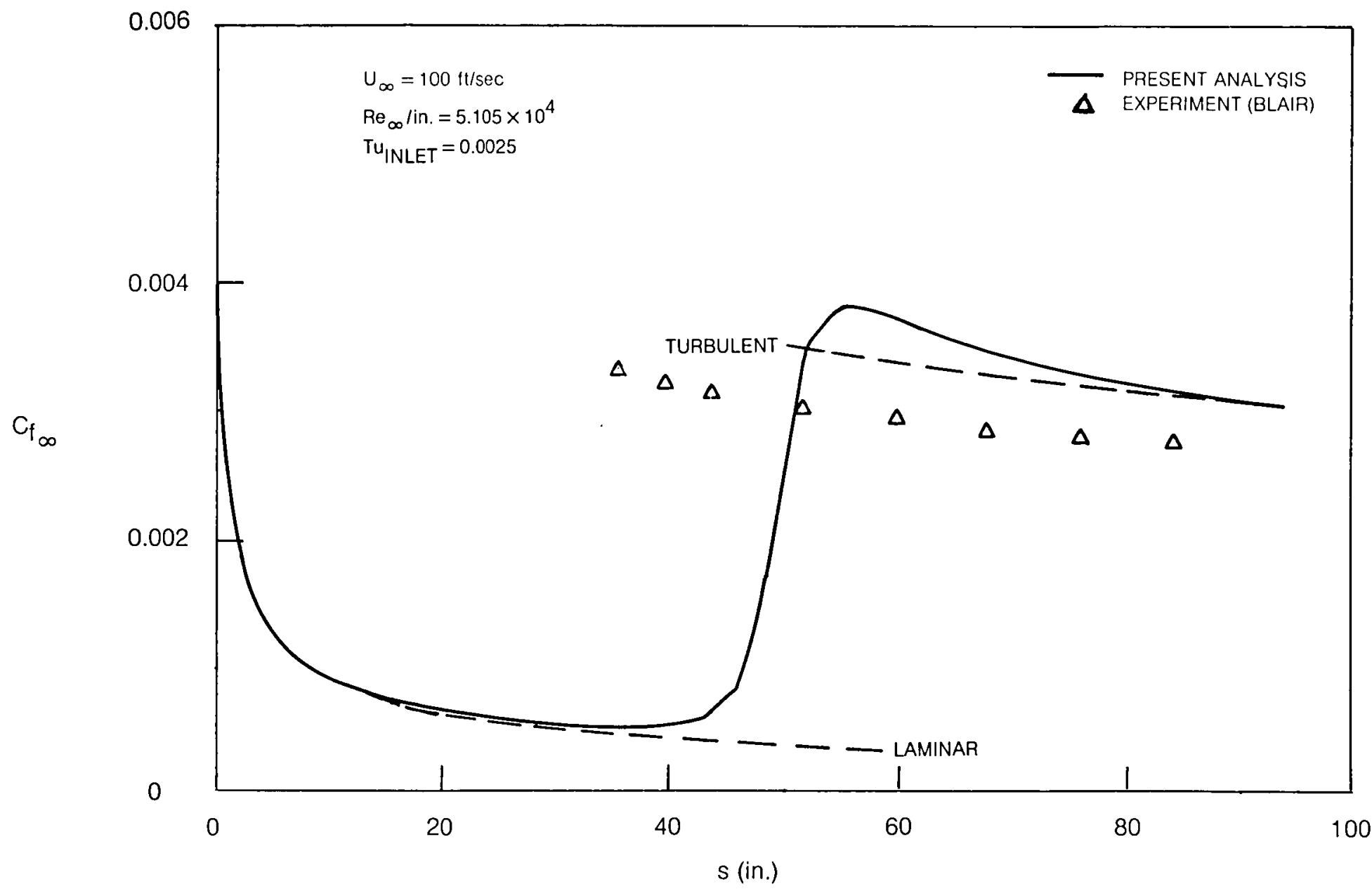

Fig. 15 Flow over flat plate at low free stream turbulence level. (a) Skin friction. 


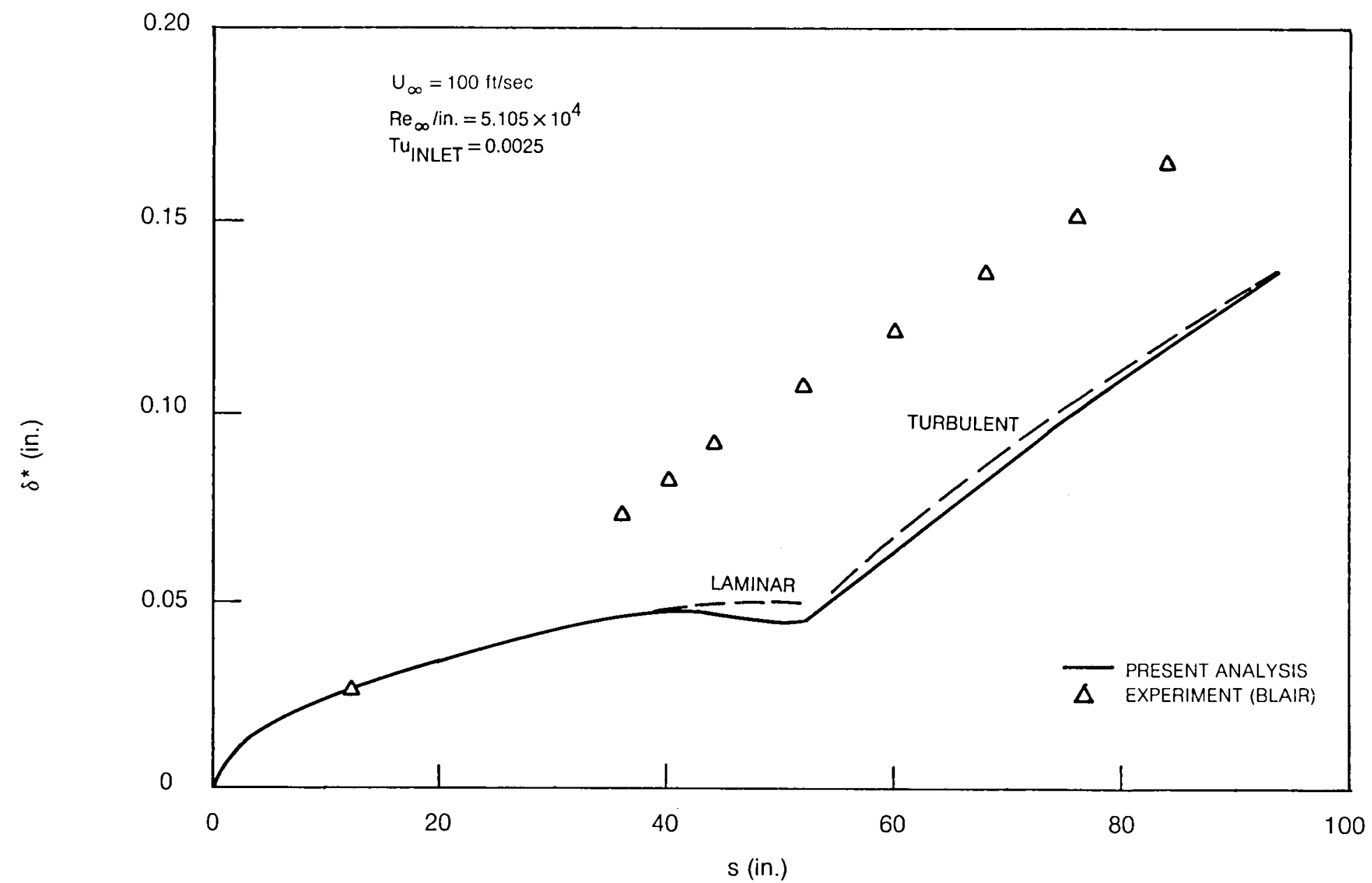

Fig. 15 Flow over flat plate at low free stream turbulence level (b) Displacement thickness 


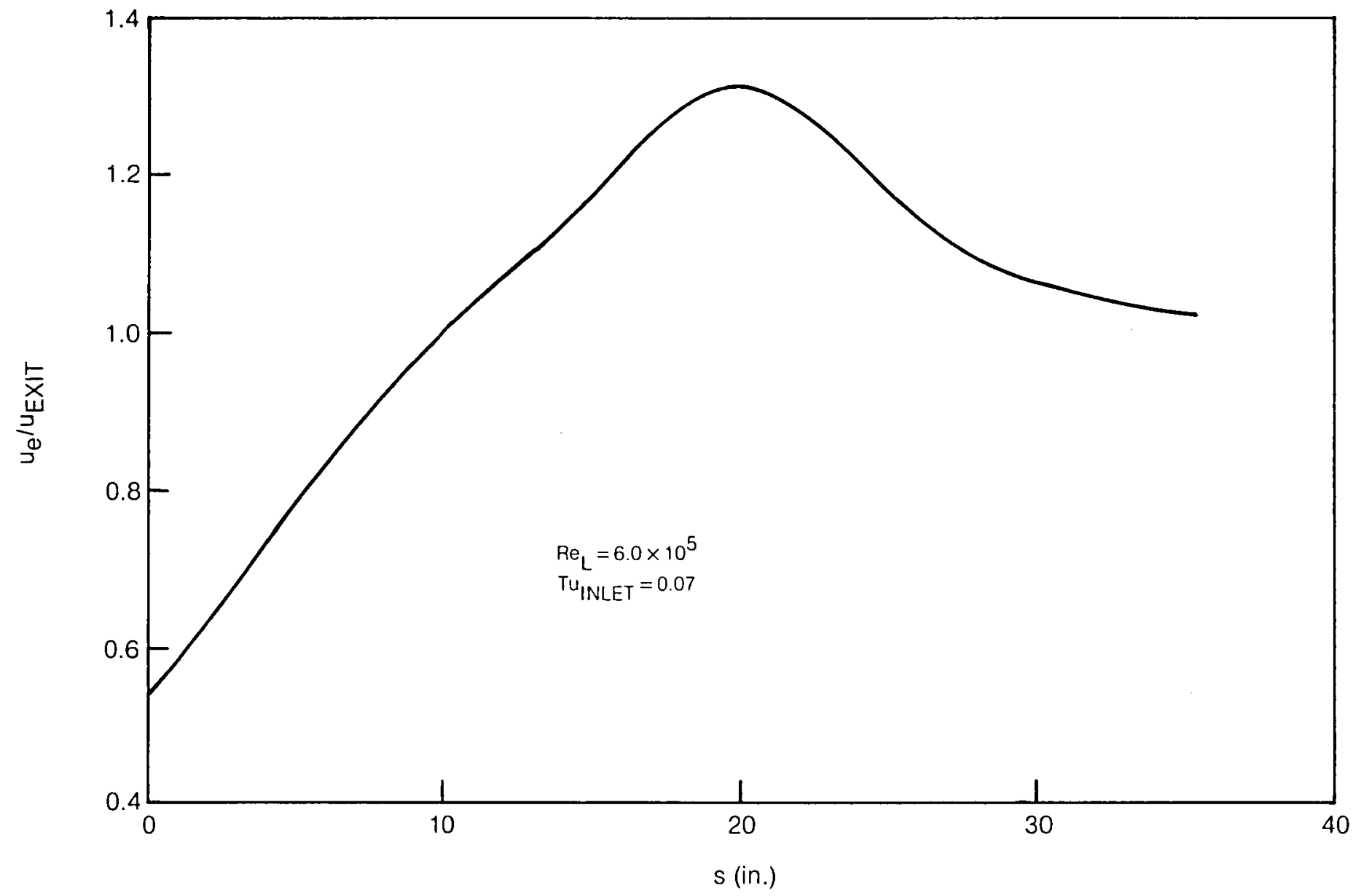

Fig. 16 Flow over flat plate with pressure gradient, high free stream turbulence level (a) Boundary layer edge velocity 


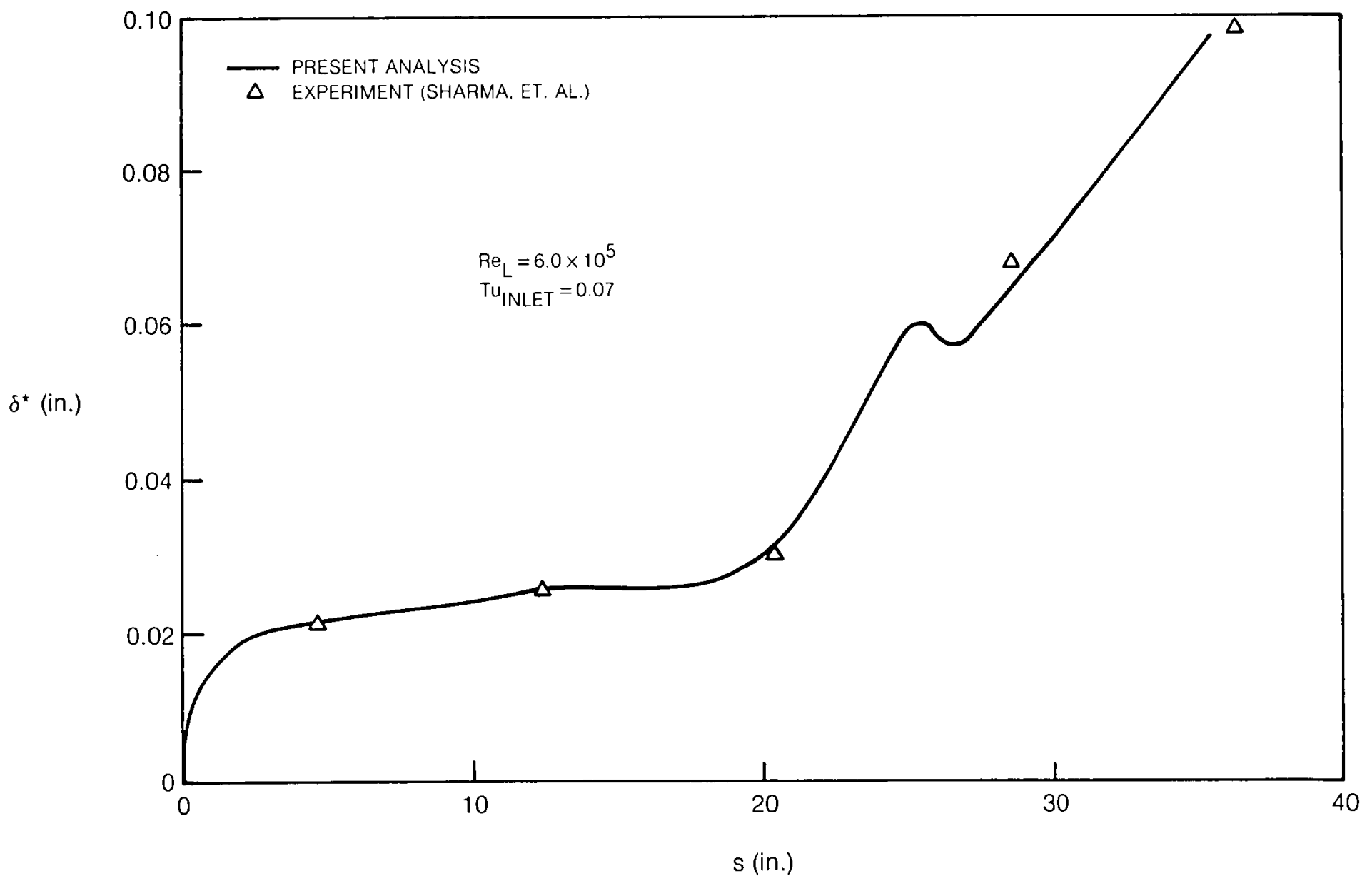

Fig. 16 Flow over flat plate with pressure gradient, high free stream turbulence level (b) Displacement thickness 


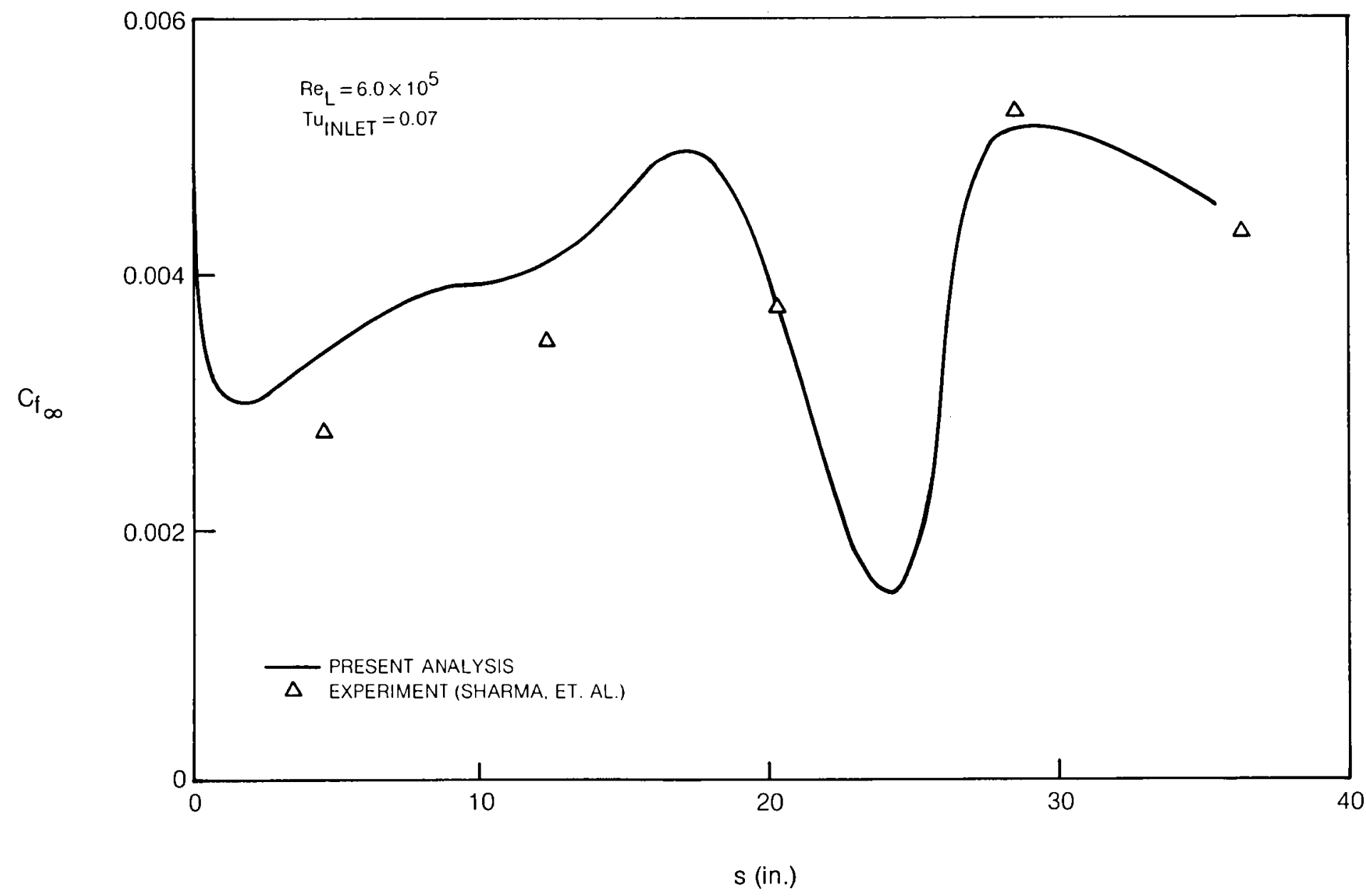

Fig. 16 Flow over flat plate with pressure gradient, high free stream turbulence level (c) Skin friction 


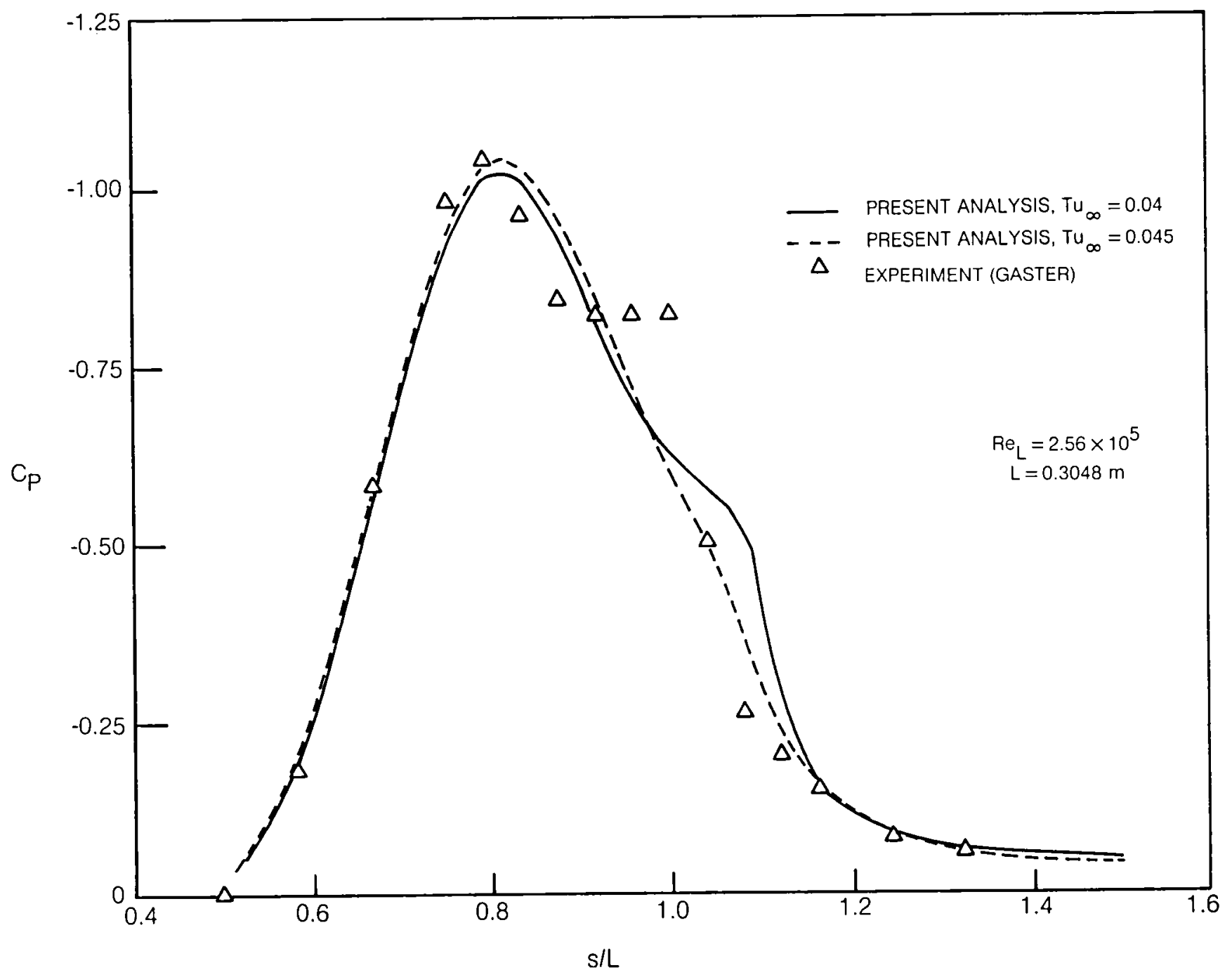

Fig. 17 Analysis of Gaster case with McDonald-Fish natural transition model. (a) Pressure distribution. 


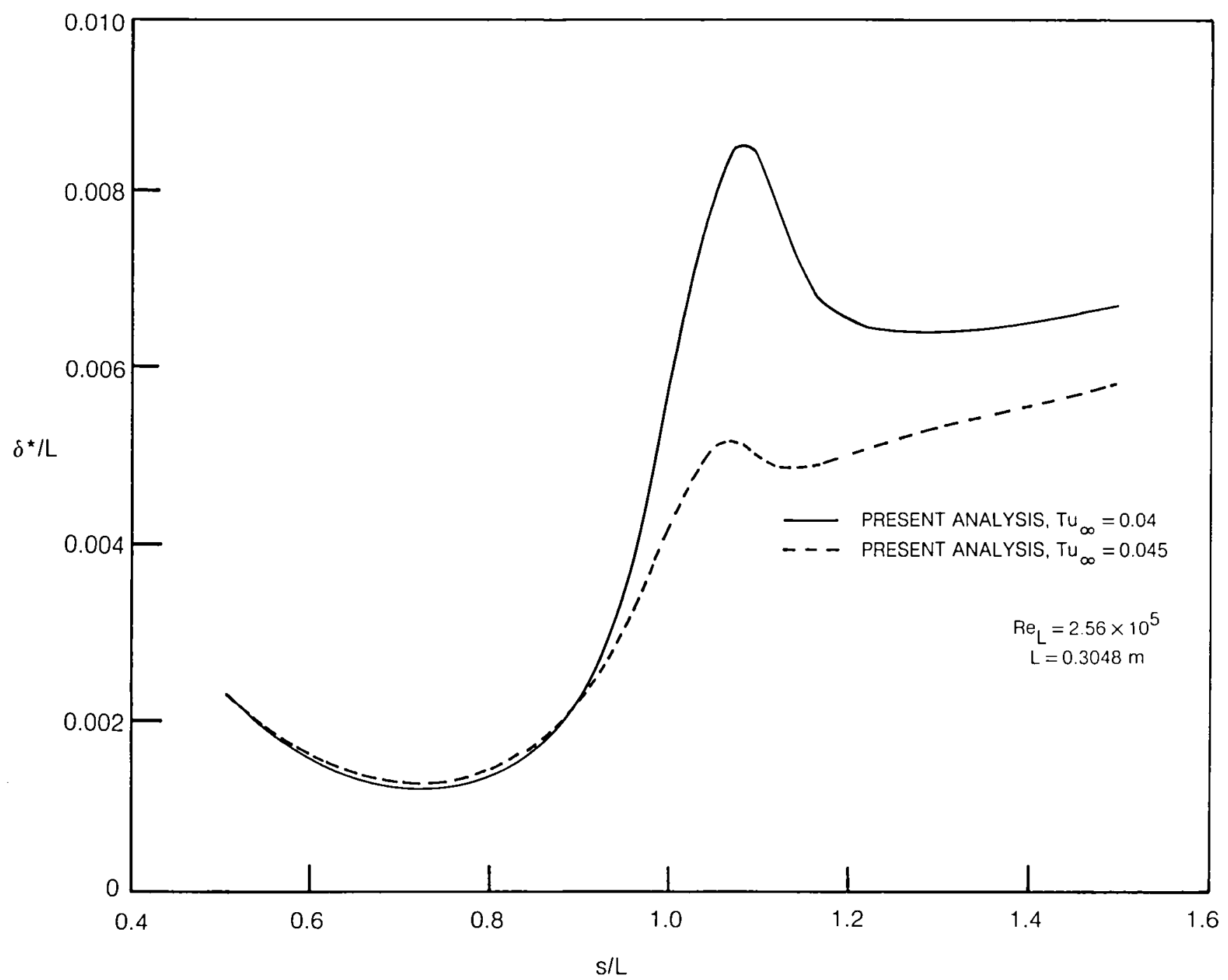

Fig. 17 Analysis of Gaster case with McDonald-Fish natural transition model. (b) Displacement thickness. 


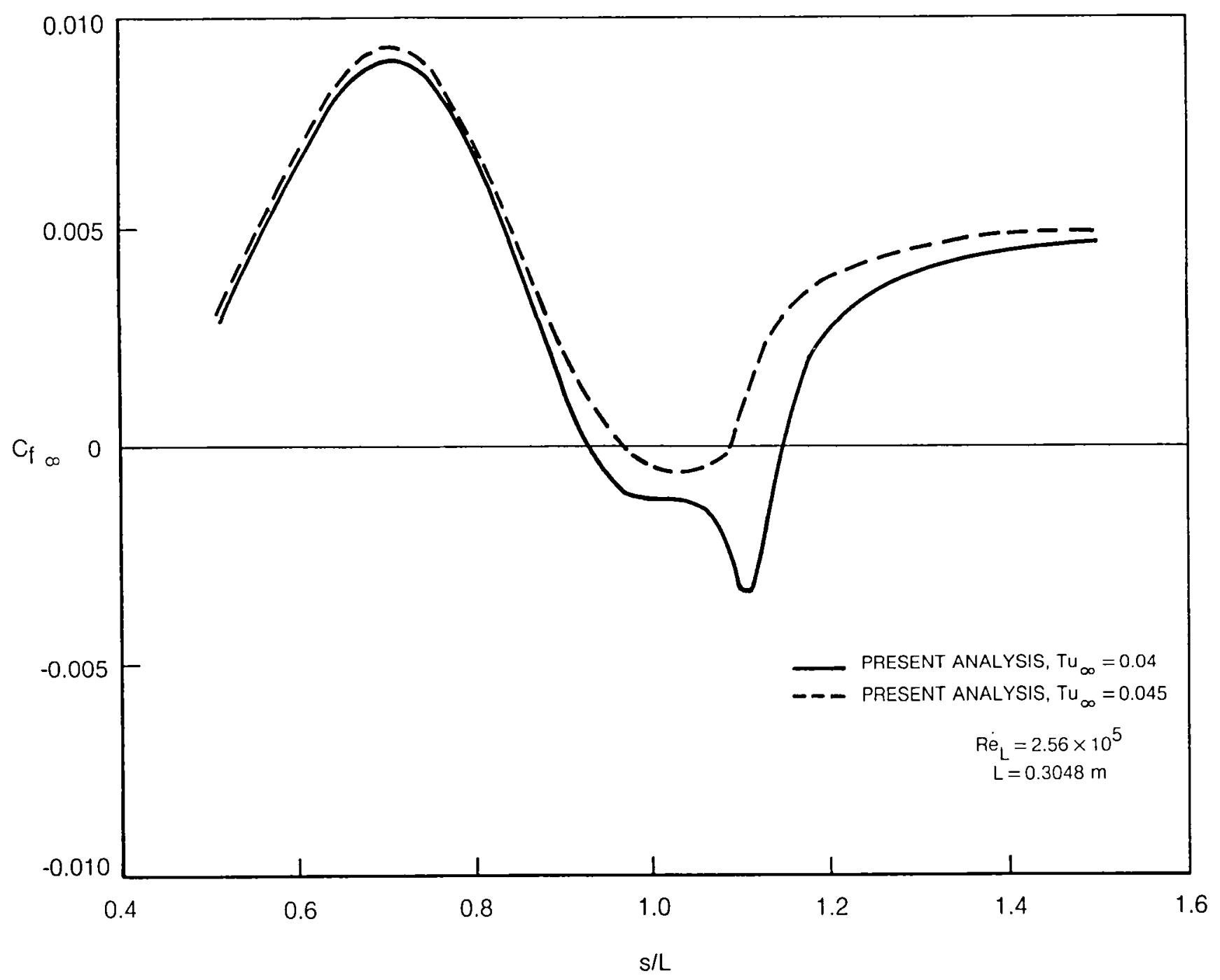

Fig. 17 Analysis of Gaster case with McDonald-Fish natural transition model. (c) Skin friction 


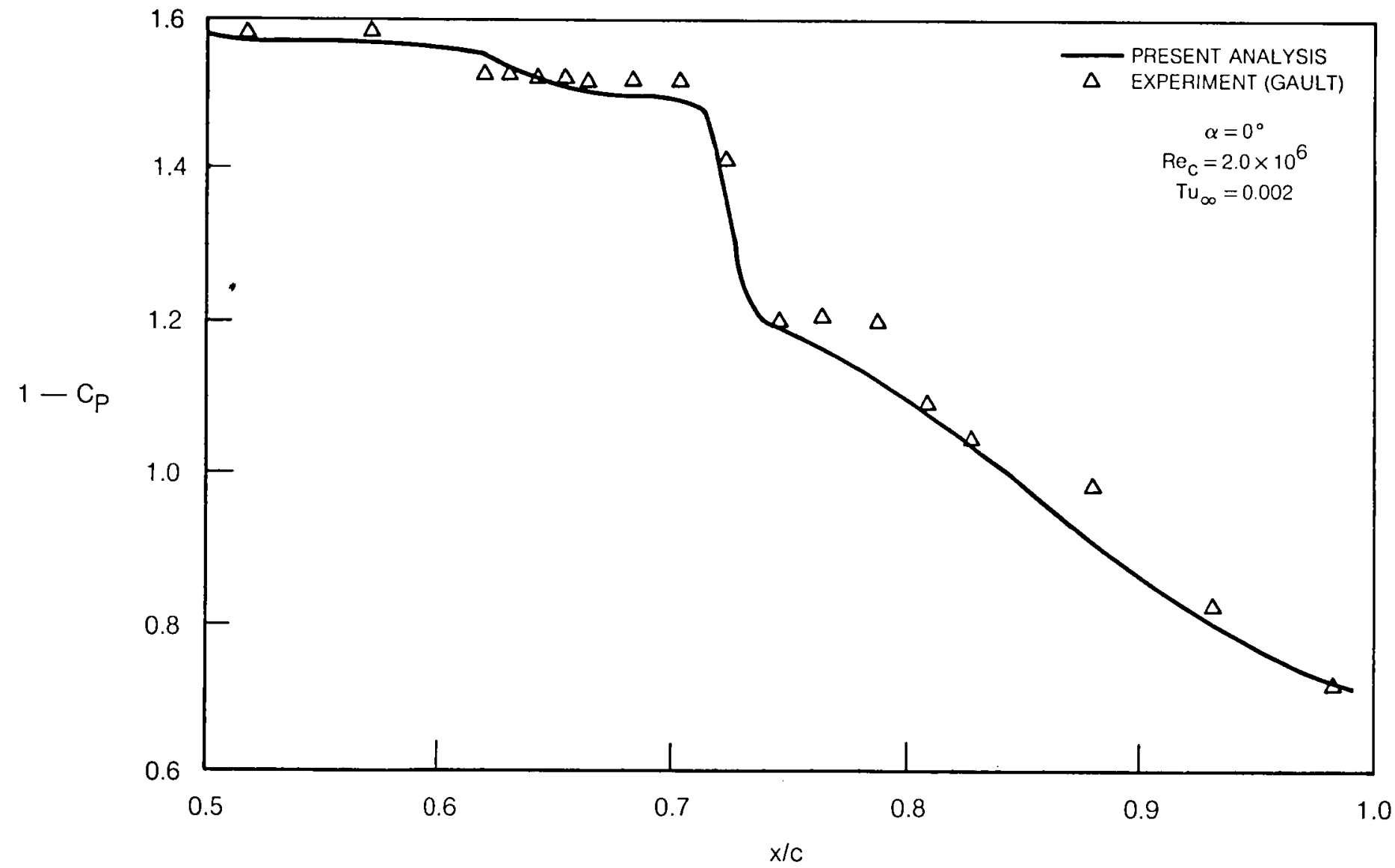

Fig. 18 Predicted results for NACA $66_{3}-018$ airfoil. (a) Pressure distribution. 


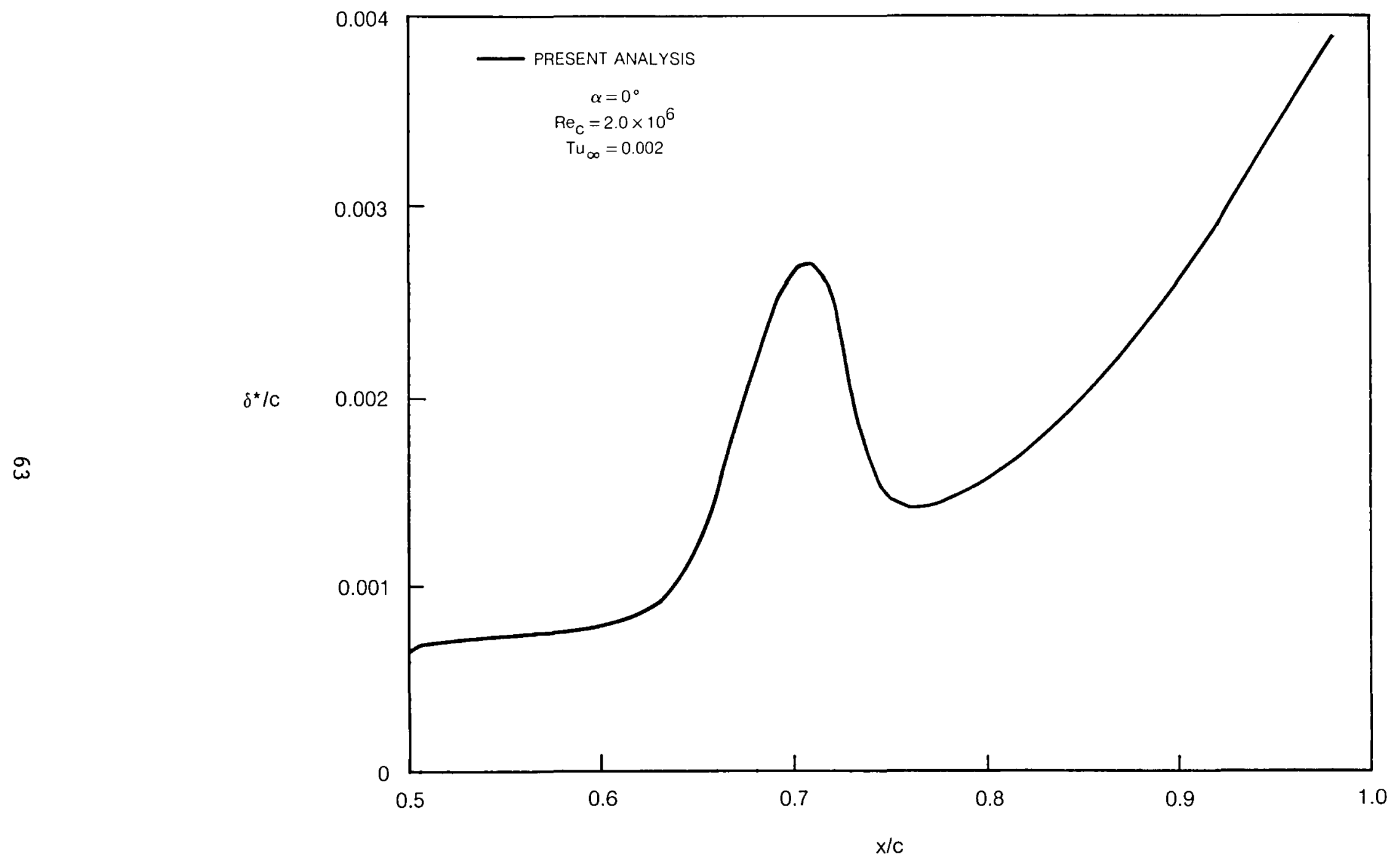

Fig. 18 Predicted results for NACA 66 3.018 airfoil (b) Displacement thickness. 


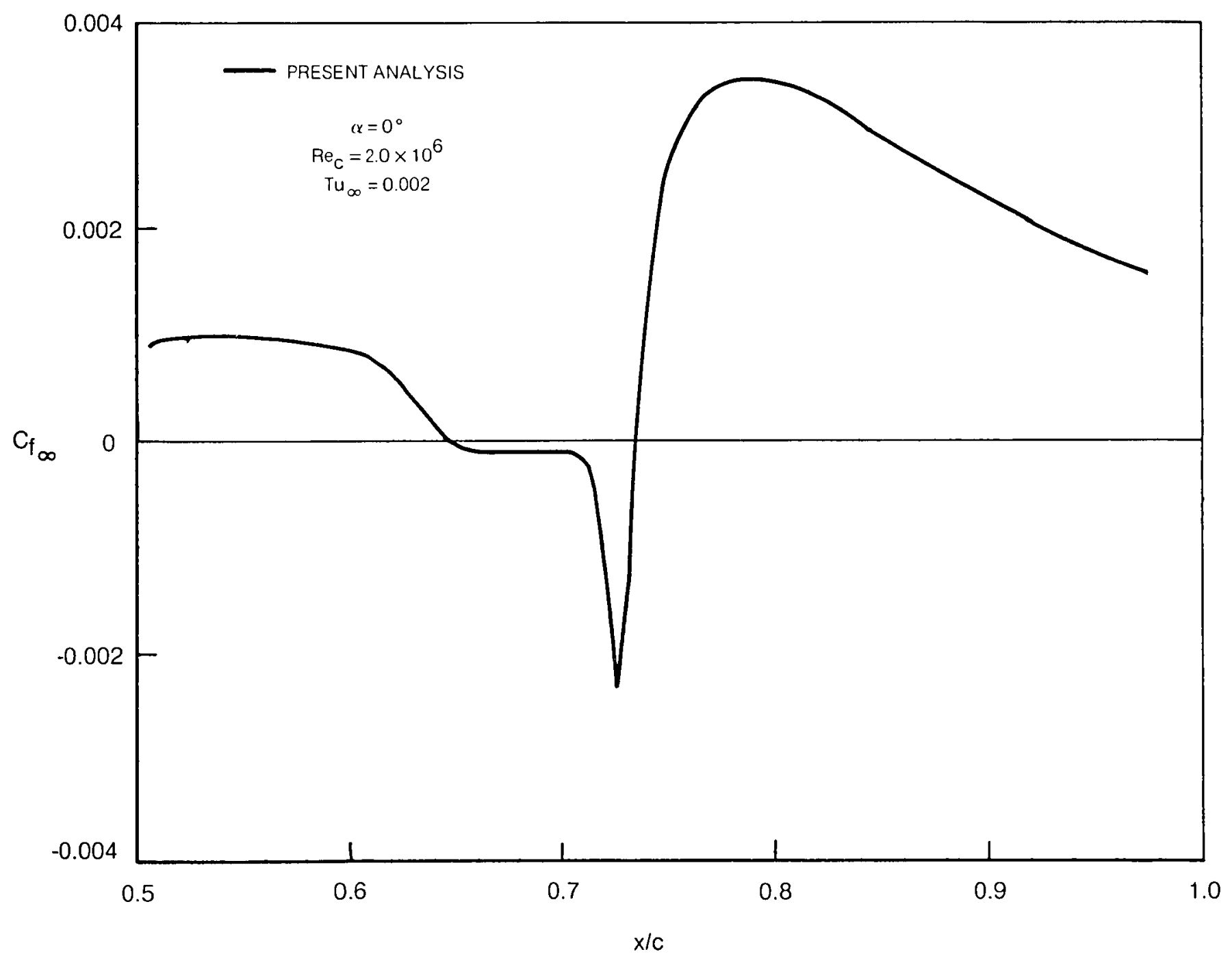

Fig. 18 Predicted results for NACA $66_{3} .018$ airfoil. (c) Skin friction. 
— FLARE

- - WINDWARD

$\triangle$ EXPERIMENT (GAULT)

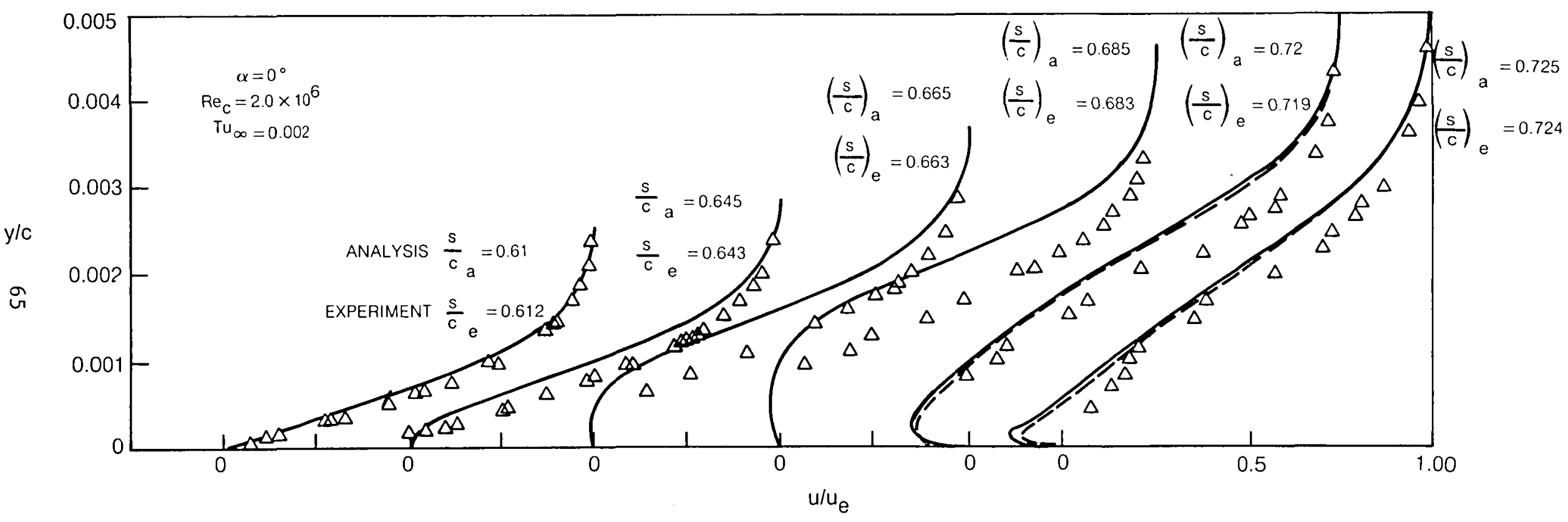

Fig. 18 Predicted results for NACA $66_{3} \cdot 018$ airfoil

(d) Velocity profiles 


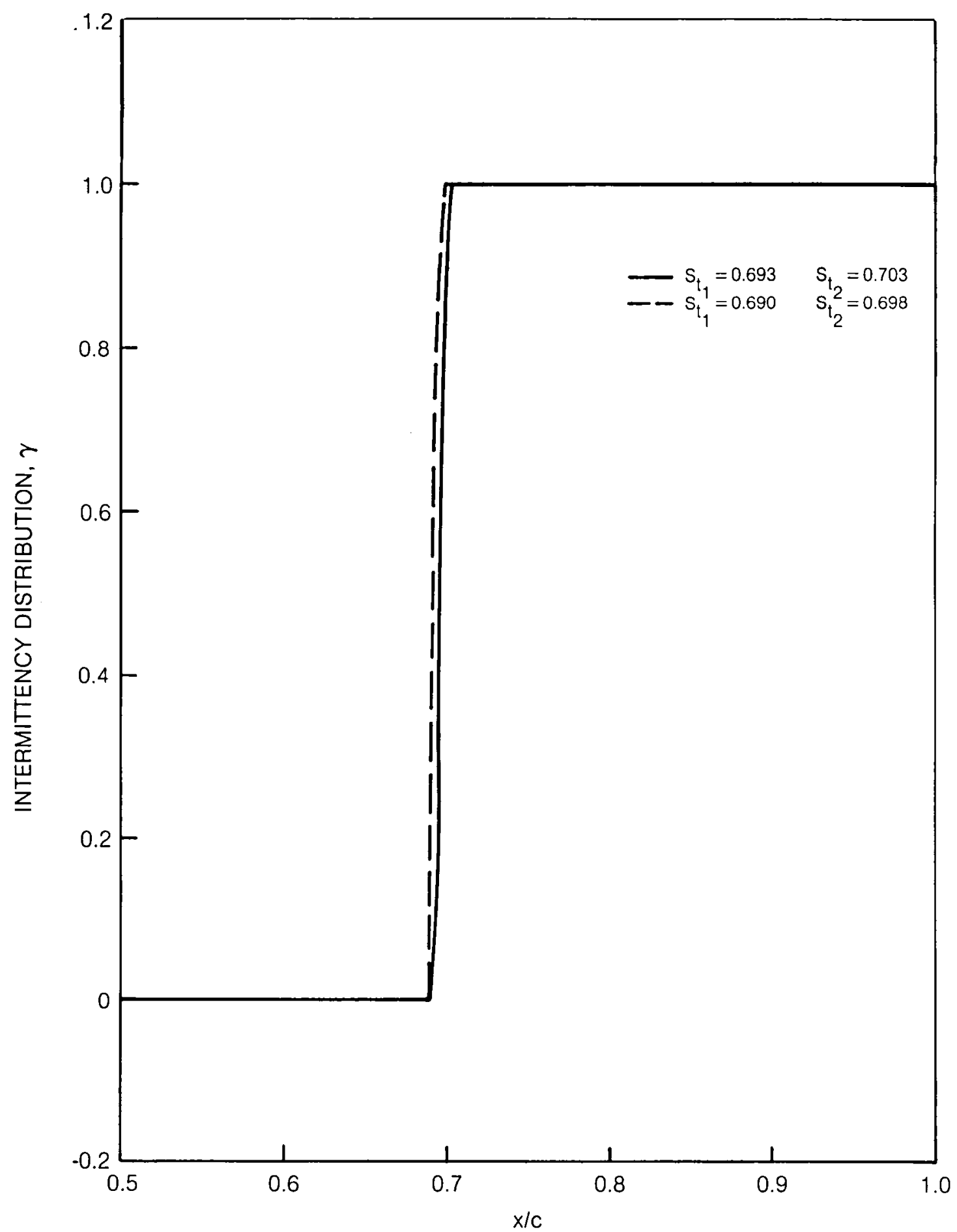

Fig. 19 Sensitivity of computed results to transition location on NACA $66_{3} .018$ airfoil (a) Imposed intermittency distributions 


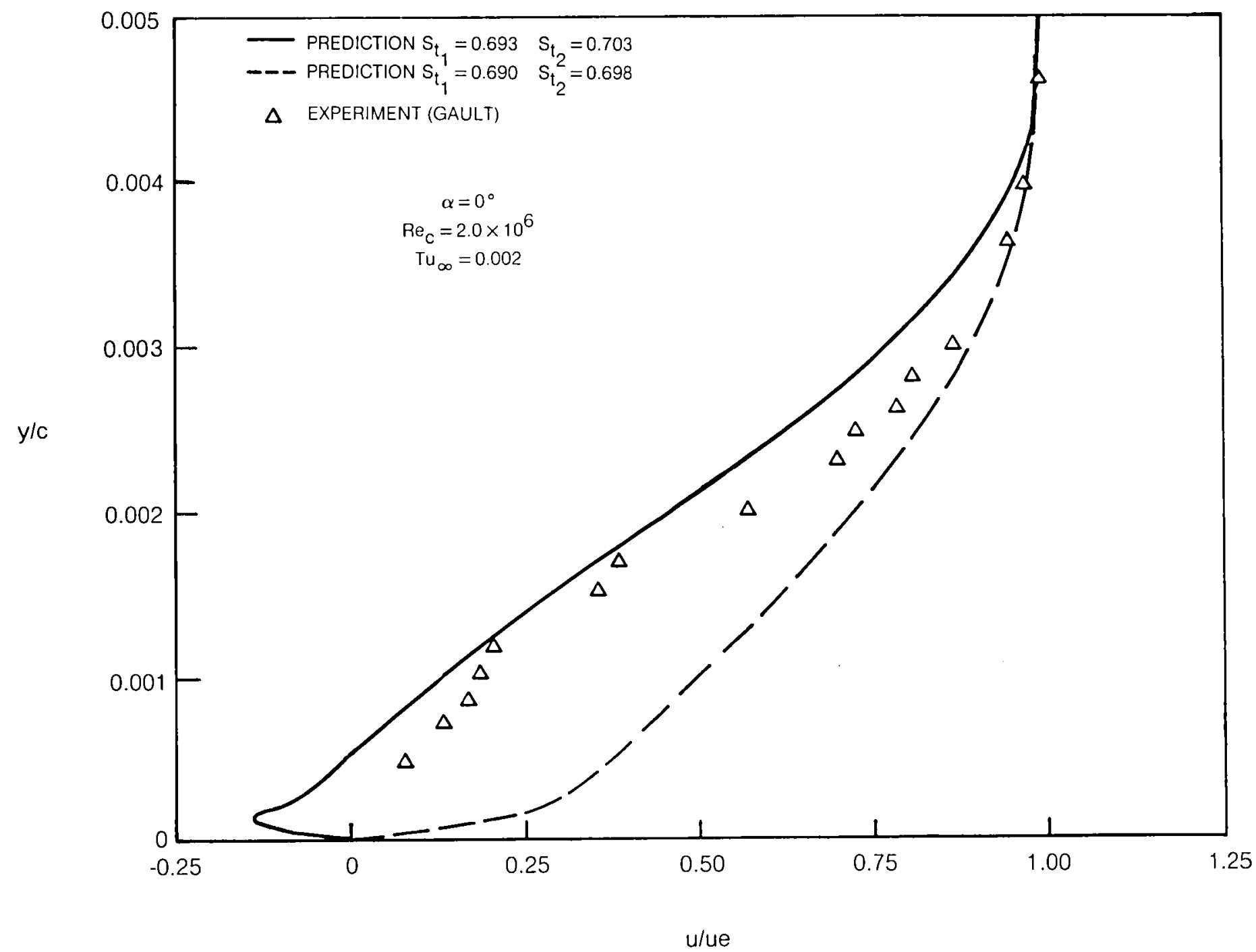

Fig. 19 Sensitivity of computed results to transition location on NACA $66_{3} \cdot 018$ airfoil (b) Velocity profiles at $s / c=0.725$ 


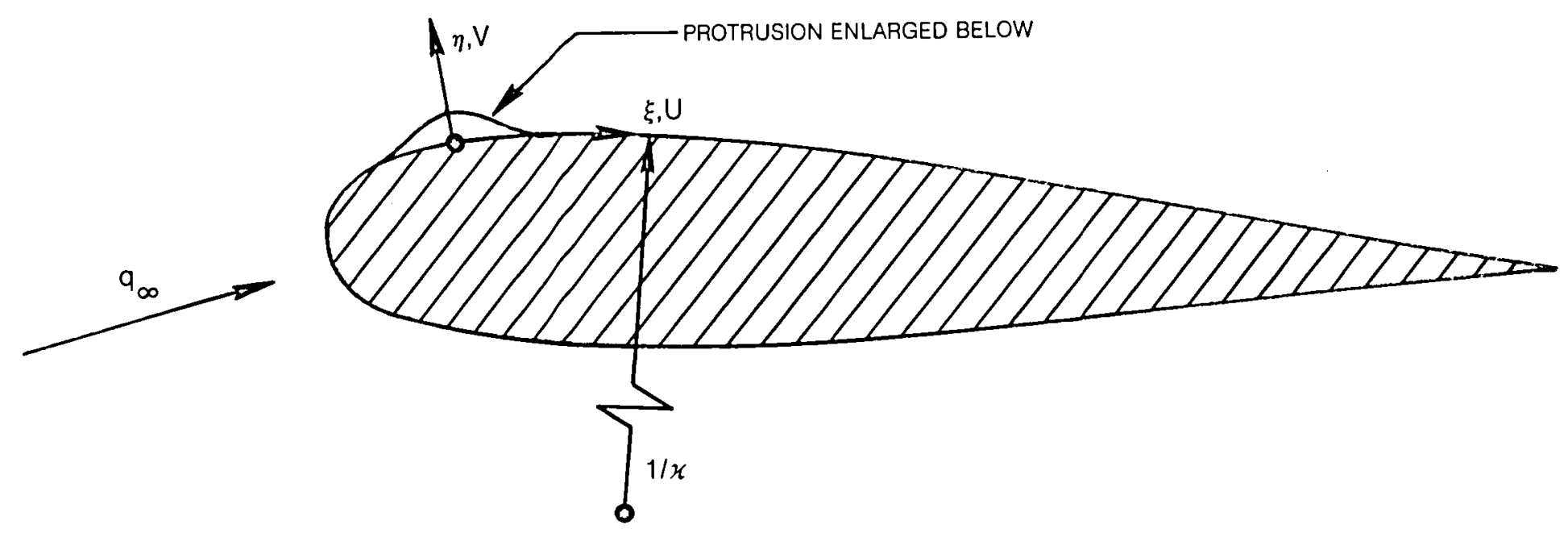

$\infty$

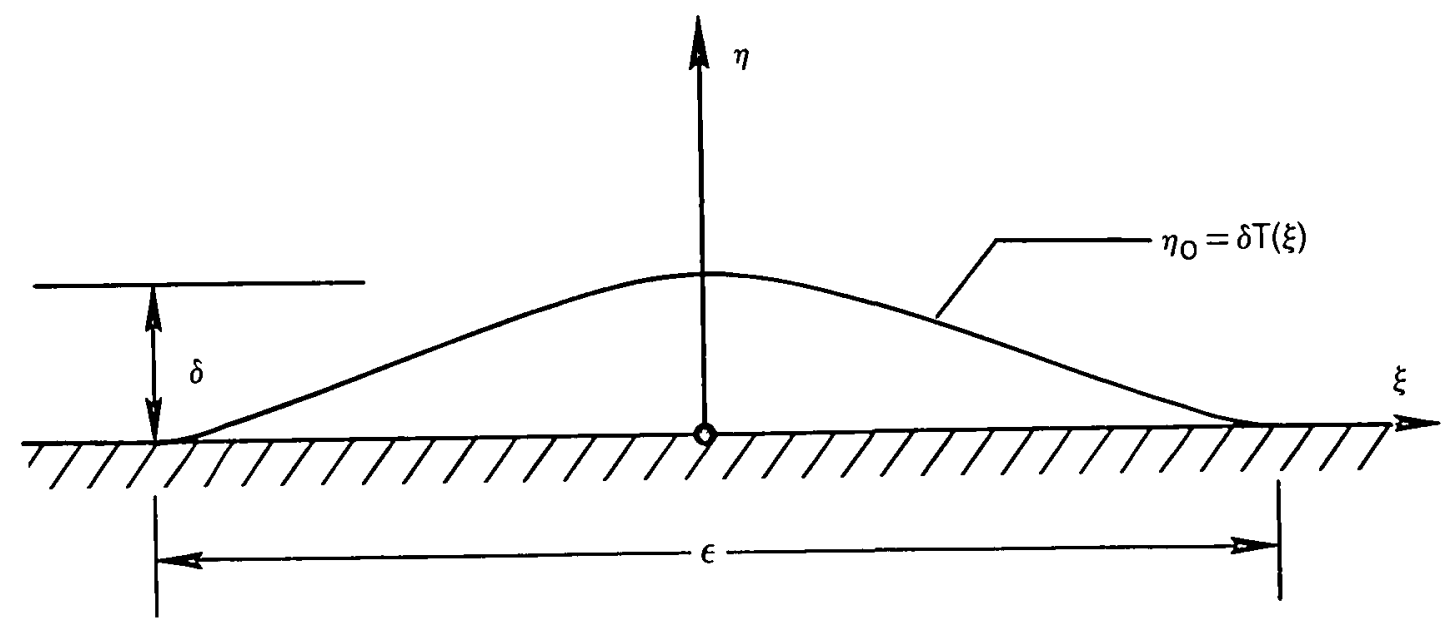

Fig. A-1 Airfoil with a Small Protrusion 





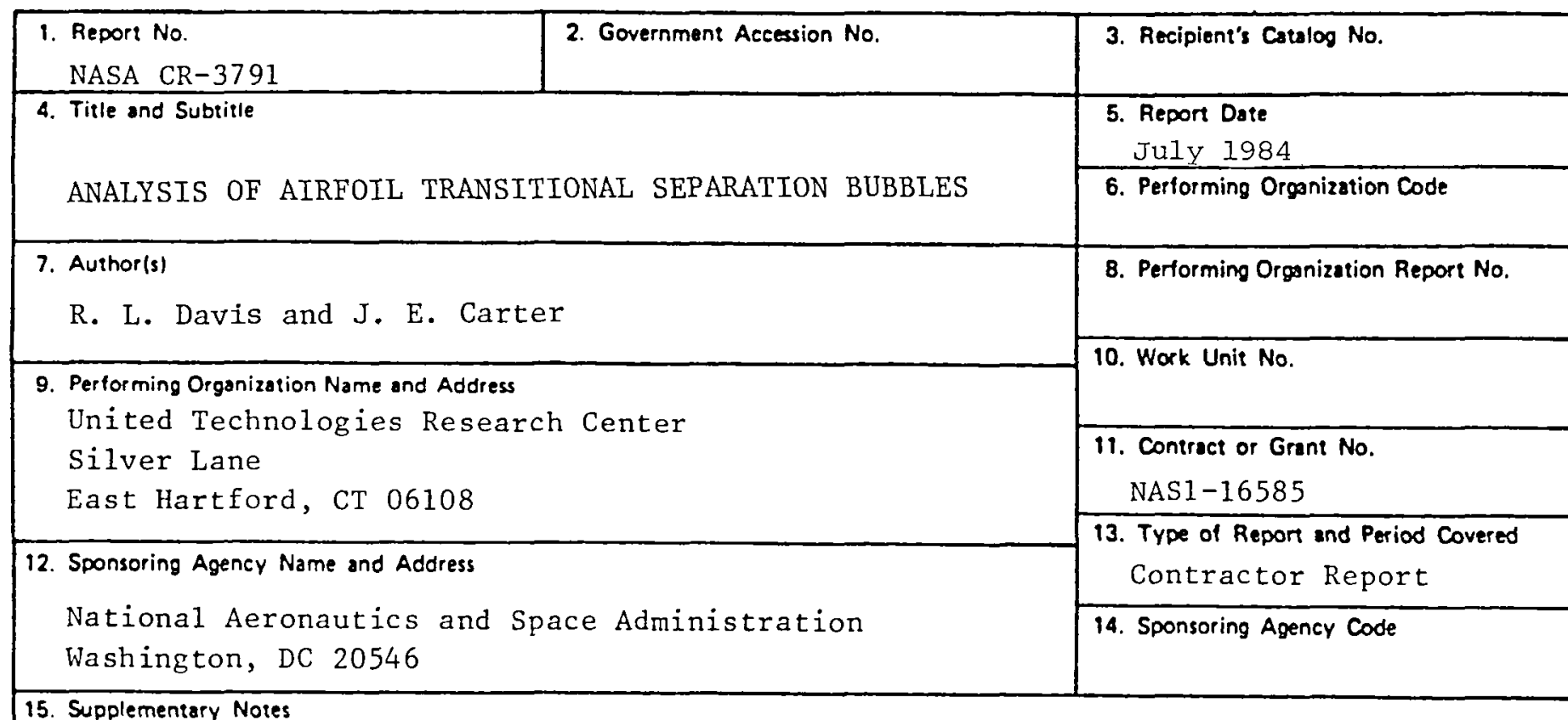

Langley Technical Monitor: Joel L. Everhart

Final Report

\section{Abstract}

A previously developed local inviscid-viscous interaction technique for the analysis of airfoil transitional separation bubbles, ALESEP (Airfoil Leading Edge Separation), has been modified to utilize a more accurate windward finite difference procedure in the reversed flow region, and a natural transition/turbulence model has been incorporated for the prediction of transition within the separation bubble. Numerous calculations and experimental comparisons are presented to demonstrate the effects of the windward differencing scheme and the natural transition/turbulence model. Grid sensitivity and convergence capabilities of this inviscid-viscous interaction technique are briefly addressed. A major contribution of this report is that with the use of windward differencing, a second, counter-rotating eddy has been found to exist in the wall layer of the primary separation bubble.

17. Key Words (Suggested by Aurhor(s))

Inviscid-Viscous, Interaction, Transitional, Separation Bubbles, Leading Edge, Airfoil
18. Distribution Statement

Unclassified - Unlimited

Subject Category 02

19. Security Cassif. (of this reportl)

Unclassified
20. Security Classif. (of this pagel

Unclassified
21. No. of Pages

71.
22. Price"

$\mathrm{AO} 4$

- For sale by the National Technical Information Service, Springfield. Virginia 22151 

National Aeronautics and Space Administration

Washington, D.C.

20546

Official Business

Penalty for Private Use, $\$ 300$
Postage and Fees Paid National Aeronautics and Space Administration NASA-451 